\title{
Architecture for Adaptive Remote Patient Monitoring
}

By

\section{Vilas Joshi, Ph.D., M.Tech., B.E.}

\author{
A thesis submitted to \\ The Faculty of Graduate Studies and Postdoctoral Affairs \\ in partial fulfilment of the degree requirements of
}

Master of Applied Science in Biomedical Engineering

(Ottawa-Carleton Institute for Biomedical Engineering (OCIBME))

Department of Systems and Computer Engineering

Carleton University

Ottawa, Ontario, Canada, K1S 5B6

May 2011

Copyright (C 2011- Vilas Joshi 
Library and Archives

Canada

Published Heritage

Branch

395 Wellington Street

Ottawa ON K1A ON4

Canada
Bibliotheqque et

Archives Canada

Direction du

Patrimoine de l'édition

395, rue Wellington

Ottawa ON K1A ON4

Canada
Your file Votre référence

ISBN: 978-0-494-81707-0

Ourfile Notre réfárence

ISBN: 978-0-494-81707-0
NOTICE:

The author has granted a nonexclusive license allowing Library and Archives Canada to reproduce, publish, archive, preserve, conserve, communicate to the public by telecommunication or on the Internet, loan, distribute and sell theses worldwide, for commercial or noncommercial purposes, in microform, paper, electronic and/or any other formats.

The author retains copyright ownership and moral rights in this thesis. Neither the thesis nor substantial extracts from it may be printed or otherwise reproduced without the author's permission.

\section{AVIS:}

L'auteur a accordé une licence non exclusive permettant à la Bibliothèque et Archives Canada de reproduire, publier, archiver, sauvegarder, conserver, transmettre au public par télécommunication ou par l'Internet, prêter, distribuer et vendre des thèses partout dans le monde, à des fins commerciales ou autres, sur support microforme, papier, électronique et/ou autres formats.

L'auteur conserve la propriété du droit d'auteur et des droits moraux qui protège cette thèse. $\mathrm{Ni}$ la thèse ni des extraits substantiels de celle-ci ne doivent être imprimés ou autrement reproduits sans son autorisation.
In compliance with the Canadian Privacy Act some supporting forms may have been removed from this thesis.

While these forms may be included in the document page count, their removal does not represent any loss of content from the thesis.
Conformément à la loi canadienne sur la protection de la vie privée, quelques formulaires secondaires ont été enlevés de cette thèse.

Bien que ces formulaires aient inclus dans la pagination, il n'y aura aucun contenu manquant. 
The undersigned recommend to

The Faculty of Graduate Studies and Postdoctoral Affairs

acceptance of the thesis

\title{
Architecture for Adaptive Remote Patient Monitoring
}

\author{
Submitted by \\ Vilas Joshi, Ph.D., M.Tech., B.E. \\ in partial fulfilment of the requirements for \\ the degree of Master of Applied Science in Biomedical Engineering
}

Chair, Dr. H. M. Schwartz, Department of Systems and Computer Engineering

Thesis Supervisor, Dr. Rafik Goubran

Carleton University

May 2011 


\section{Abstract}

The population is aging at an unprecedented rate around the world and the majority of the aging population suffers from chronic illnesses like heart disease, stroke, diabetes, respiratory diseases, and cancers in addition falls are major cause of mortality with elderly patient

Remote patient monitoring offers improved care to patients with chronic and/or acute illnesses in their place of residence without the need for frequent visits to the hospital, thus reducing the medical cost as well as improving the quality of life for the patients. A remote patient monitoring system architectural design is proposed that is cost effective, robust and scalable so that components can be added as plug-and-play modules.

The proposed architecture was extended to include an adaptive information content management method and buffering scheme to reduce the loss of patient data and improve survivability of the monitoring system under adverse network conditions like network congestion and/or temporary loss of network connectivity.

A remote patient monitoring system to monitor bed occupancy using a pressure sensitive mat was implemented and a trial was conducted to verify its application in the field. A usability study was done to evaluate the user response to a graphical user interface (GUI) prototype for the bed occupancy remote patient monitoring system. Using the feedback from this usability study, the functional GUI was implemented. The application of an adaptive information content management method for the bed occupancy monitoring system was demonstrated and the improvement in the survivability of the system was evaluated. 


\section{Acknowledgements}

I would like to express my deepest gratitude to my supervisor Dr. Rafik Goubran whose direction, guidance and support made this thesis possible. Throughout this thesis work he provided suggestions and encouragements that were invaluable.

I would like to thank the Natural Sciences and Engineering Research Council of Canada (NSERC) for their financial assistance as well as the Department of Systems and Computer Engineering and Carleton University.

I am indebted to many of my colleagues for providing a stimulating and fun environment in which to learn and grow. I am especially grateful to Hanieh Chatrzarrin, Payam Moradshahi, Omid Abari, Melanie Pouliot, Megan Holtzman and Amaya Arcelus for friendship and for listening to me patiently even when I went off tangent.

My special thanks also go to Marina Gobrial for her help and input with matlab GUI design.

Finally I would like to say thanks to my son, Sameer, for love, encouragement and keeping me grounded. 


\section{Table of Contents}

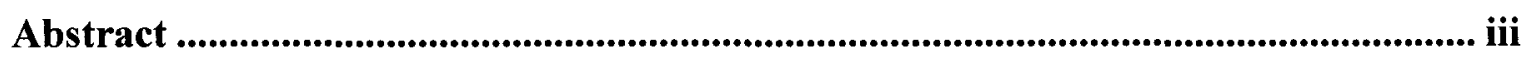

Acknowledgements .............................................................................................................................iv

Table of Contents.....................................................................................................................................

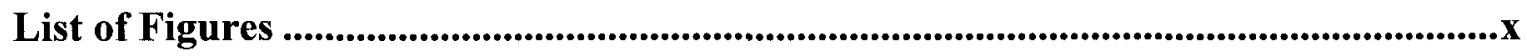

List of Abbreviations ................................................................................................... xiii

Chapter 1: Introduction ..................................................................................................................1

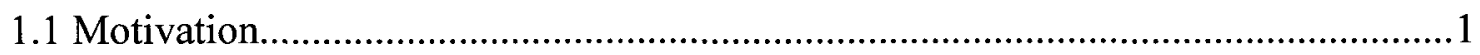

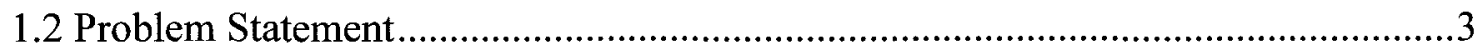

1.3 Thesis Objective and Scope

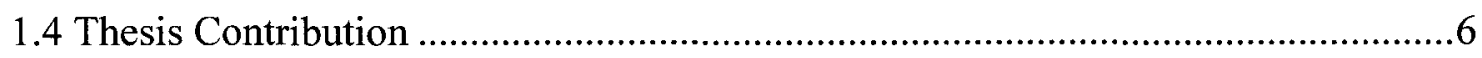

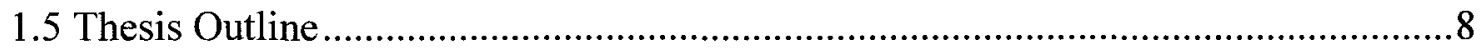

Chapter 2: Remote Patient Monitoring System Design Considerations .......................10

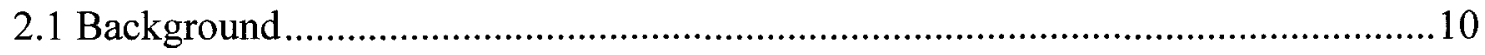

2.2 RPM Systems in mHealth.......................................................................................12

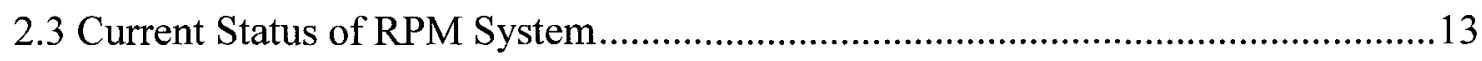

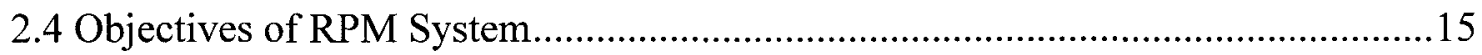

2.5 Signals Monitored by RPM Systems........................................................................18

2.6 Remote Patient Monitoring System Components ………........................................20

2.7 RPM System Architecture Design Criteria ................................................................23

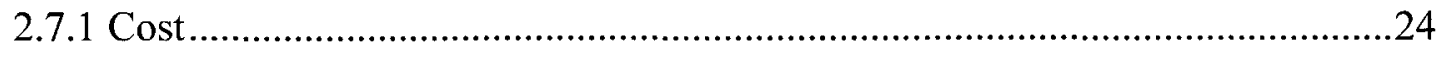

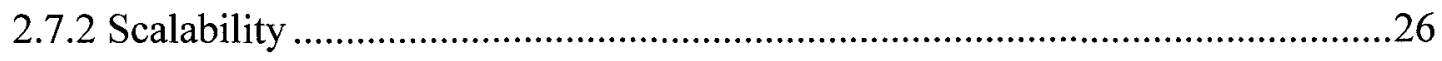

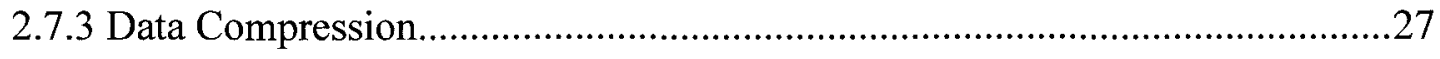




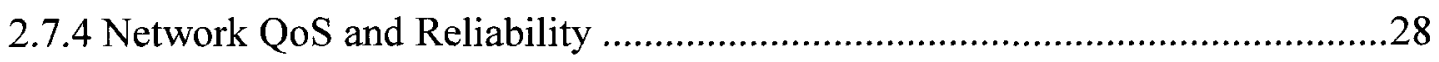

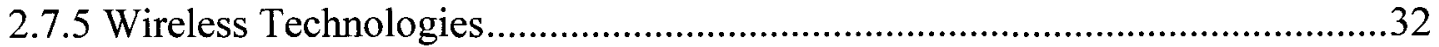

2.7.5.2 Wireless Sensor Network Technologies for RPM.....................................32

2.7.5.2 Wireless Broadband Technology for RPM .................................................37

2.7.6 Data Security and Privacy .............................................................................38

2.7.7 Real-Time Signal Processing......................................................................42

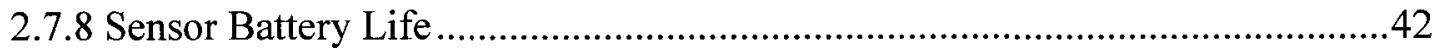

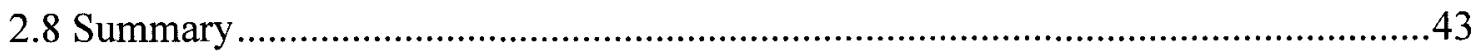

Chapter 3: Current RPM System Architecture.............................................................45

3.1 Distributed Data Processing and Storage Architecture: ……...........................46

Chapter 4: Proposed RPM System Architecture.......................................................................49

4.1 Proposed Centralized Data Processing and Storage Architecture for the RPM

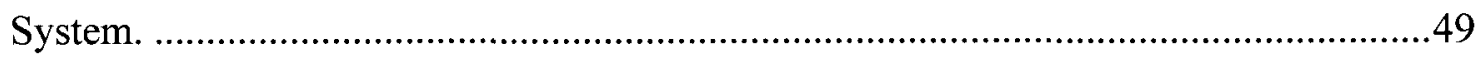

4.2 Comparison of Distributed and Centralized Architecture ..................................53

4.2.1 Network BW and QoS Requirements........................................................54

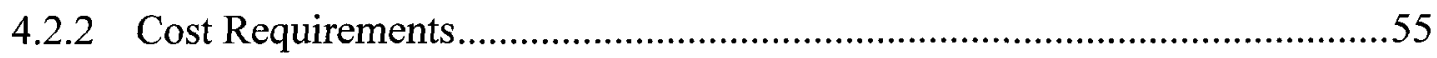

4.2.3 Scalability and Flexibility ........................................................................56

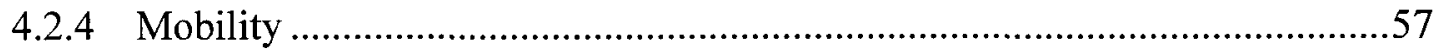

4.2.5 Cloud Computing and Proposed RPM System Architecture..........................57

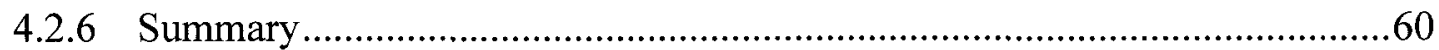

Chapter 5: Proposed Adaptive Information Content Management and Buffer

Caching Design .....................................................................................................................62

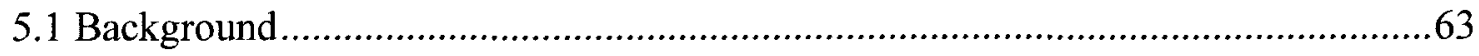

5.2 Proposed Adaptive Information Content Management (AICM) Method..................64

5.2.1 RPM System Architecture using the AICM and Buffer Caching ....................64

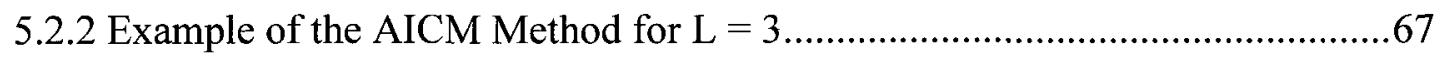

5.2.3 Survivability Interval (SI) Evaluation for Different $\mathrm{D}_{2}$ and $\mathrm{D}_{3} \ldots \ldots \ldots \ldots \ldots \ldots \ldots \ldots \ldots . . . .70$

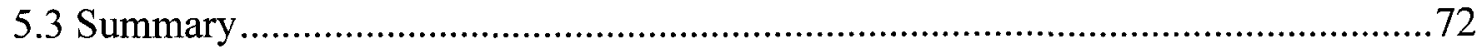




\section{Chapter 6: Proposed Contiguous Buffering Scheme for the AICM to Maximize}

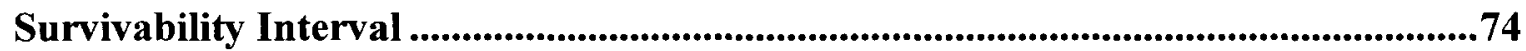

6.1 Example of Proposed Contiguous Buffering scheme Functionality for $L=3 \ldots \ldots \ldots .77$

6.1.1 SI Evaluation for Contiguous Buffering Schemes ...................................81

6.2 Comparison of the AICM system using Static vs. Contiguous Buffering Scheme.83

6.2.1 Choice of Number of Levels for Static and Contiguous Buffering Schemes...87

6.3 Performance of the AICM Method during Network Congestion ........................89

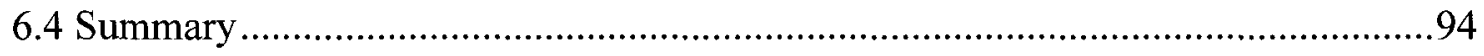

\section{Chapter 7: Implementation of an Bed Occupancy RPM System Using Pressure}

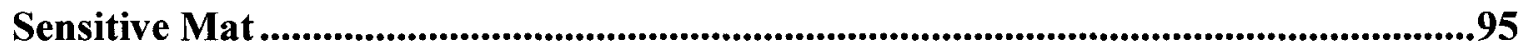

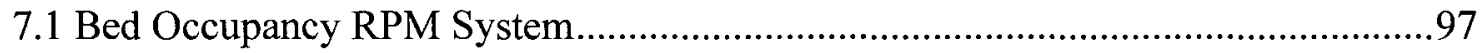

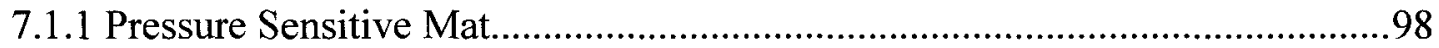

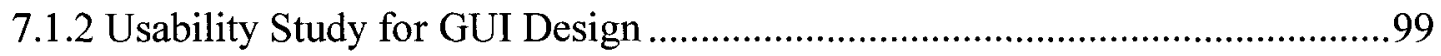

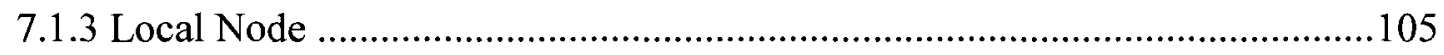

7.1.4 Trial of Bed Occupancy RPM System ............................................... 106

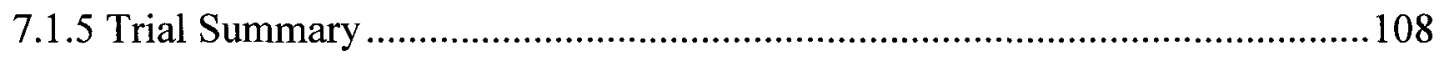

7.2 Proposed Centralized Data Processing and Storage Architecture for Bed

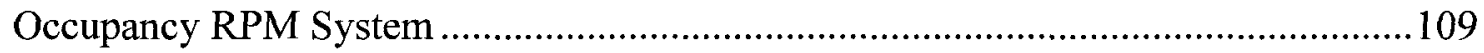

7.3 Case Study: Application of the AICM to Bed Occupancy RPM System .............111

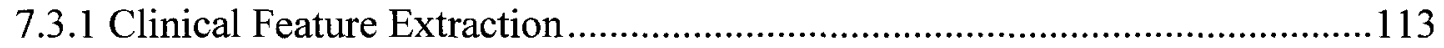

7.3.2 Content Reduction Method.................................................................... 115

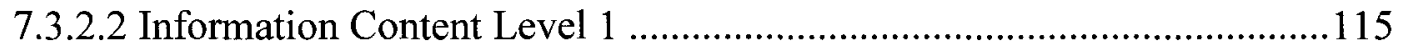

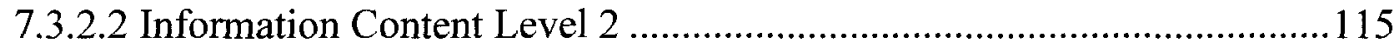

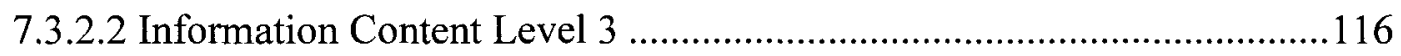

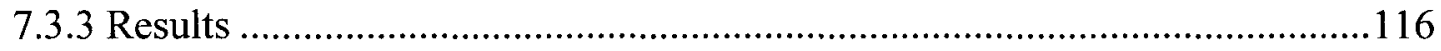

7.3.3.2 System Survivability................................................................... 116

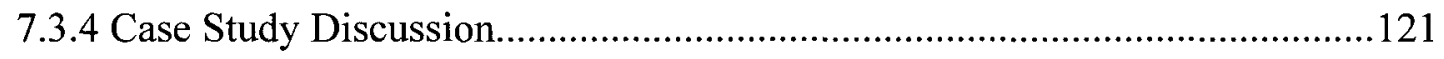

Chapter 8: Discussion...........................................................................................123

8.1 Adaptive Information Content Reduction ................................................ 123 
8.1.1 Techniques for Information Content Reduction

Chapter 9: Conclusions ...............................................................................................127

9.1 Summary of Results and Contributions ..................................................... 127

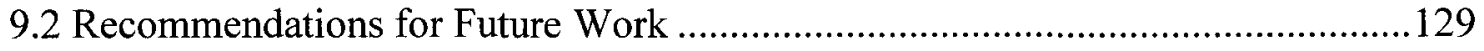

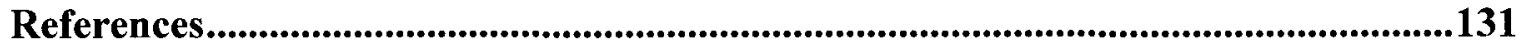




\section{List of Tables}

Table 2.1: Commercially available RPM Systems ............................................................14

Table 2.2: List of Biological Signals commonly monitored using RPM systems.............19

Table 2.3: List of Environmental signals commonly monitored using RPM systems. .....20

Table 2.4: Typical sensors currently used along with their data rate and real-time requirements.

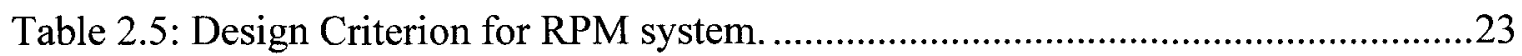

Table 2.6: Wireless Sensor Technology Protocols.............................................................35

Table 2.7: Maximum data rate available over different wireless networks........................38

Table 3.1: Sensors and Data Transfer Rate Requirements. .............................................45

Table 4.1: Comparison of the RPM system using Distributed and Centralized Data

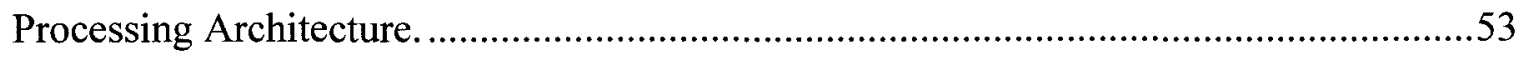

Table 6.1: Comparison of Static and Contiguous Buffering Scheme for $\left(D_{2}=100\right.$ and $D_{3}$

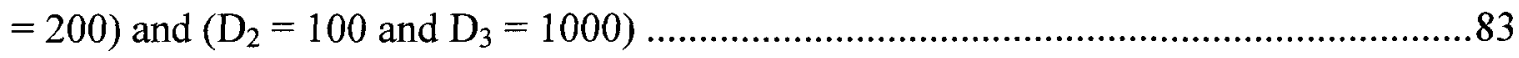

Table 7.1: Information Retained, Rate Reduction, and Survivability at each Level .......118

Table 7.2: Standard Deviation of Features at Level 2 and 3 compared to Level 1 .........119 


\section{List of Figures}

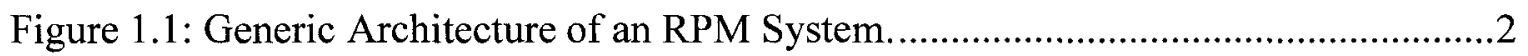

Figure 2.1: Power consumption, data rate and range are the three key considerations when selecting a wireless interface reproduced from: [96], [97] ..............................................36

Figure 3.1: Distributed Data Processing and Storage Architecture ....................................46

Figure 4.1: Centralized Data Processing and Storage Architecture .................................50

Figure 4.2: Model of Centralized Data Processing RPM System. .....................................52

Figure 4.3: Cloud Computing Model, reproduced from: [126].......................................58

Figure 4.4: Centralized Data Processing RPM System using Cloud Computing..............59

Figure 5.1: AICM RPM system using static multi buffering scheme. ..............................66

Figure 5.2: Adaptive information content and static multi buffer RPM system with little

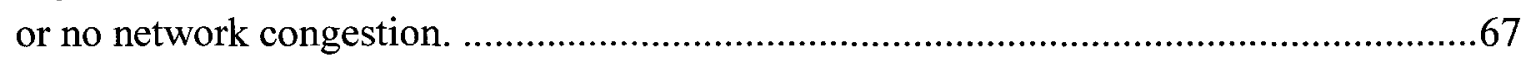

Figure 5.3: Adaptive information content and static multi buffer RPM system with

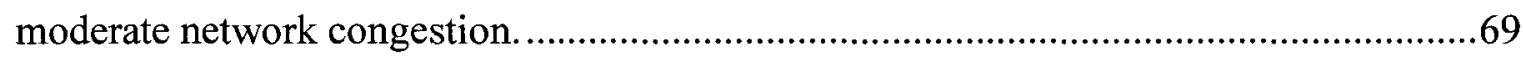

Figure 5.4: Adaptive information content and static multi buffer RPM system with heavy

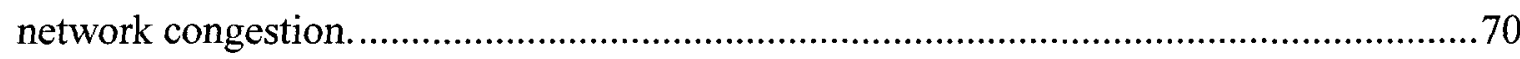

Figure 5.5: Comparison of Survivability Interval for the RPM system with and without

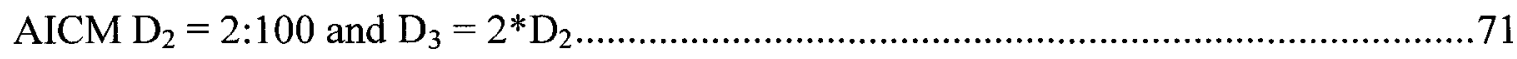

Figure 5.6: Comparison of Survivability Interval for the RPM system with and without

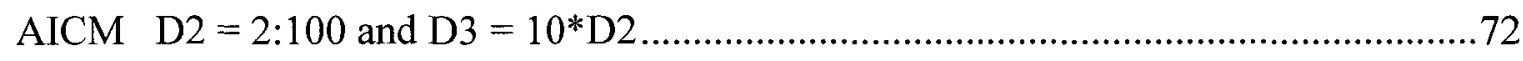

Figure 6.1: Data flow for AICM RPM system using contiguous buffering scheme.........75

Figure 6.2: Adaptive information content and contiguous buffering RPM system with no or little network congestion.

Figure 6.3: Adaptive information content and contiguous buffering RPM system with

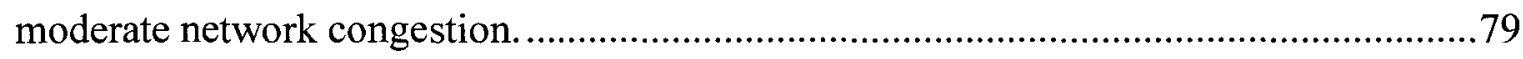

Figure 6.4: Adaptive information content and contiguous buffering RPM system with or heavy network congestion. .80 
Figure 6.5: Comparison of Survivability Interval for Contiguous Buffering RPM system

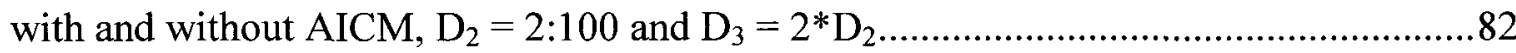

Figure 6.6: Comparison of Survivability Interval for Contiguous Buffering RPM system

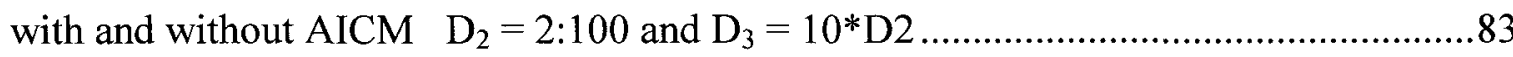

Figure 6.7: Comparison of Survivability Interval for Static (a) and Contiguous (b) Buffering RPM systems with no AICM and 3-Level AICM with D2=2 and D3=5. .84

Figure 6.8: Comparison of Survivability Interval for Static (a) and Contiguous (b) Buffering RPM systems with no AICM and 3-Level AICM with D2 $=10$ and D3 $=100 \ldots 85$

Figure 6.9: Comparison of Survivability Interval for Static (a) and Contiguous (b) Buffering RPM systems with no AICM and 2-Level AICM with D2=10.

Figure 6.10: Comparison of Survivability Interval for Static (a) and Contiguous (b) Buffering RPM systems with no AICM and 2-Level AICM with D2=100.

Figure 6.11: Comparison of $\mathrm{SI}_{\mathrm{st}}$ for 2-Level and 3-Level static buffering system for same information content reduction $\mathrm{D}=100$. .88

Figure 6.12: Comparison of $\mathrm{SI}_{\mathrm{co}}$ for 2-Level and 3-Level contiguous buffering system for same information content reduction $\mathrm{D}=100$. .88

Figure 6.13: Plot of adaptive information content management RPM system under different network congestion contiguous buffering scheme.

Figure 6.14: Overflow time in seconds for 3-Level AICM system with $D_{2}=50$ and $D_{3}=$ 100 .

Figure 6.15: Overflow time in seconds for 3-Level AICM system with $\mathrm{D}_{2}=5$ and $\mathrm{D}_{3}=$ 10 .

Figure 7.1: Block diagram of Bed Entry/Exit RPM System.

Figure 7.2: Different pressure mat set up used for bed entry/exit RPM system................99

Figure 7.3: Legend location and size for the homepage on Version A..........................101

Figure 7.4: Legend location and size for the homepage on Version B ..........................102

Figure 7.5: Bed activity tabs in Version A, Level-2 _......................................................103

Figure 7.6: Bed activity tab in Version B, Level-2......................................................104

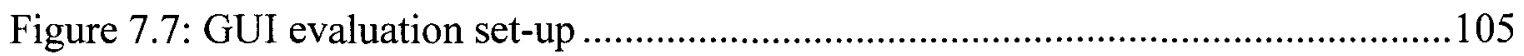


Figure 7.8: GUI for bed entry/exit RPM system, showing weekly bed occupancy data. 107

Figure 7.9: GUI for bed occupancy RPM system, showing monthly view of bed occupancy data.

Figure 7.10: Bed Occupancy RPM System utilizing Centralized Data Processing Architecture.

Figure 7.11: Application of AICM method to Bed Occupancy RPM System.

Figure 7.12: Information extraction.

Figure 7.13: Comparison of survivability interval for bed occupancy RPM system using conventional buffering vs. one using AICM system.

Figure 7.14: Bed exit times at all levels.

Figure 7.15: Respiratory rates at all three levels 120 


\section{List of Abbreviations}

$\begin{array}{ll}\text { AICM } & \text { Adaptive Information Content Management } \\ \text { ATM } & \text { Asynchronous Transfer Mode } \\ \text { BAN } & \text { Body Area Network } \\ \text { EHR } & \text { Electronic Health Record } \\ \text { GUI } & \text { Graphical User Interface } \\ \text { IR } & \text { Infra Red } \\ \text { MPLS } & \text { Multiprotocol Label Switching } \\ \text { PDA } & \text { Personal Digital Assistant } \\ \text { QoS } & \text { Quality of Service } \\ \text { RPM } & \text { Remote Patient Monitoring } \\ \text { SI } & \text { Survivability Interval } \\ \text { TAFETA } & \text { Technology Assisted Friendly Environment for the Third Age } \\ \text { UWB } & \text { Ultra Wide Band } \\ \text { WPAN } & \text { Wireless Personal Area Network } \\ \text { WSN } & \text { Wireless Sensor Network }\end{array}$




\section{Chapter 1: Introduction}

\subsection{Motivation}

The population is aging around the globe at an unprecedented rate. The UN report on the aging population states that "By 2050, the number of older persons (60+years) in the world will exceed the number of young for the first time in history." [1]. The health of a person typically deteriorates as they age. An aging population will have significant impact on health care spending and costs. Elderly people are more likely to suffer from chronic diseases like, congestive heart failure, diabetes, Chronic Obstructive Pulmonary Disease (COPD) and chronic wounds or skin ulcers.

In the last decade and especially in last 5 years there has been enormous interest and research in technologies for Remote Patient Monitoring (RPM), telemonitoring, homecare, smart homes and eHealth. This has been facilitated by the advances in cheaper hardware, sensor technology, Wireless Personal Area Network (WPAN) technology (Bluetooth, Zigbee etc), the Internet and increased bandwidth available over wireless broadband networks.

Innovative approaches to chronic health care services are needed to reduce the burden on already stretched resources and to keep the cost of care manageable. Telehealth technologies, specifically the RPM technologies, are one such innovation [2]. 
The RPM systems collect and monitor patient status in an environment outside of hospital or supervised medical facility. Monitoring of patient data involves the use of medical and non-medical sensors, software and hardware for collecting, processing and transmitting data/results to the remote healthcare provider. The data/results can be transmitted continuously or periodically as well as in real-time or via store and forward method. These data/results are then used by healthcare providers to monitor the patient's status for emergency intervention as well as trend review. Figure 1.1 shows a generic architecture of an RPM system. The patient home signifies where patient resides and nonwearable sensors are present. The connection to network from home can be wired or wireless. On the other hand wearable sensors can be monitored in home and outside using wireless technology.

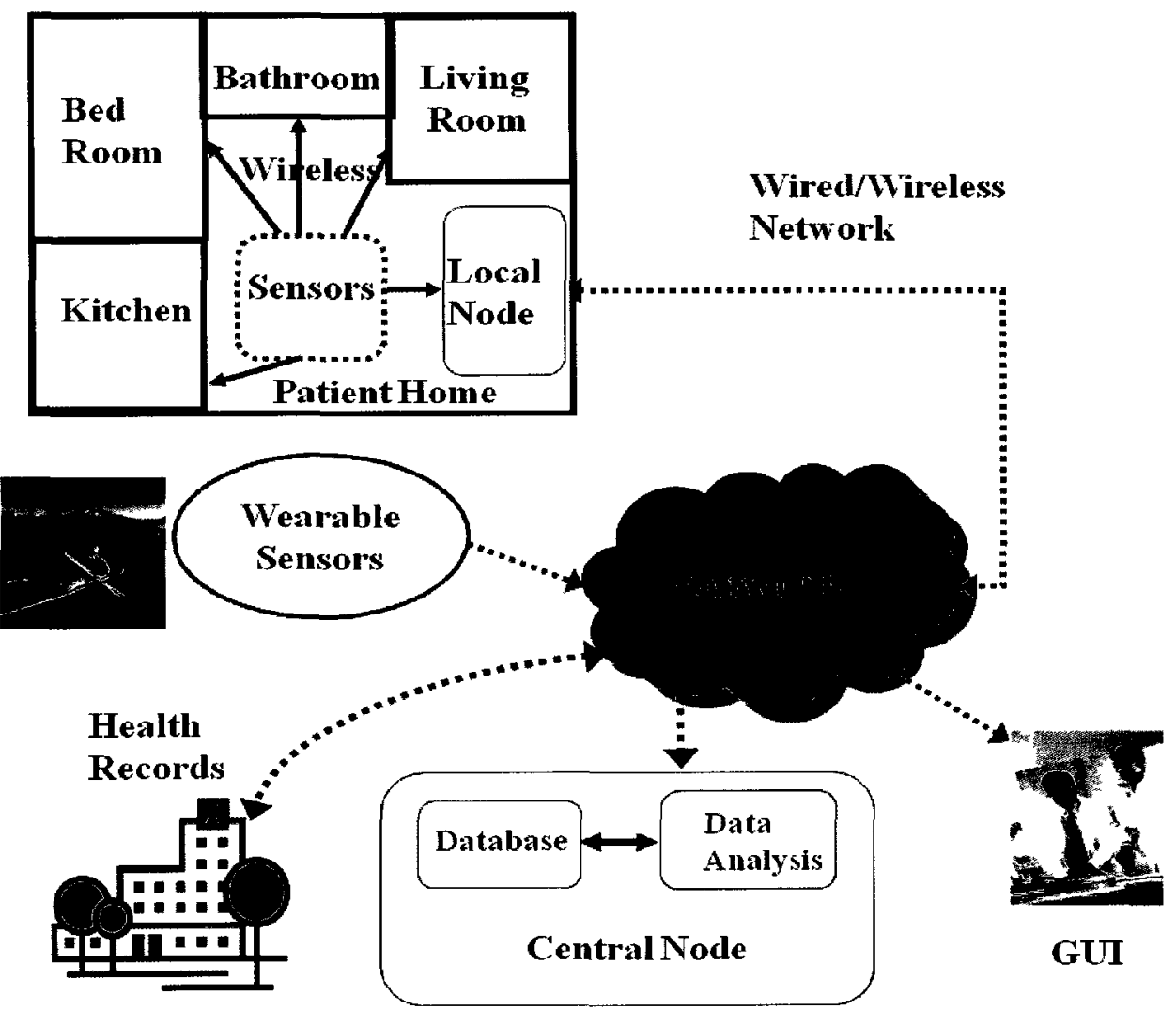

Figure 1.1: Generic Architecture of an RPM System. 
Mobile (m) Health is defined as using mobile communications devices such as PDAs and mobile phones to provide health services and information. mHealth provides a convergence of wireless communication and computing technologies to provide healthcare services. One of the strategies mHealth uses to provide healthcare services include the use of the RPM system [3].

Given the challenges of limited financial and human resources and changing demographics, the RPM systems will play a critical role in improving the quality of service for patients at a reduced cost as well as in reducing the stress and strain on healthcare providers. With high bit rate $4 \mathrm{G}$ network a feature rich and comprehensive RPM system with the ability to monitor various chronic and acute conditions becomes realizable [3].

\subsection{Problem Statement}

The driver for this thesis is the current lack of the architectural framework for the RPM systems. There is a significantly growing interest in the use of the RPM systems in healthcare. With healthcare costs and aging population, research is being widely conducted in to the RPM systems with a variety of sensors for different medical conditions, Wireless Sensor Networks (WSNs), signal processing of sensor data, Body Area Network (BAN) and data. Most of the currently proposed RPM systems monitor a set of medical conditions using different wireless sensor technologies (WSN and BAN). There is very little work done on how to integrate monitoring systems for individual biological/environmental signals in a synergetic and unified RPM system. Lack of standardization for the RPM systems has lead to systems that are incompatible due to different technologies used as well as differences in their architecture. They cannot be 
integrated in to a comprehensive RPM system, thus interoperability of different RPM systems is not guaranteed.

There is very little work being done in the area of overall architecture of the RPM system. Unless a generic architectural model for the RPM systems is defined too many heterogeneous models of the RPM systems will exist leading to limited penetrations due to incompatibility, restricted scope and even obsolescence due to constantly evolving technology in the network and computing field.

If the RPM systems are to achieve wider penetration architectural design of the RPM systems capable of monitoring a variety of medical conditions as well as being flexible enough to integrate newer sensors, wireless technologies and providing scalability so that newer sensors/processors/software can be added as plug-and-play modules is critical.

\subsection{Thesis Objective and Scope}

This thesis addresses the area of architectural framework for an RPM system. The RPM system architecture needs to be forward looking to take advantage of the newer wireless technologies like $4 \mathrm{G}$ and above which offer significantly higher network bandwidth than before as well as proliferation of smartphones. It also must be able to integrate well with the newer computing paradigm shift like cloud computing.

Wireless networks suffer from network congestion during peak hours as well as temporary loss of connectivity for short duration. While no RPM system can survive catastrophic network failure, the RPM system architecture needs to be robust and ensure that component failures, network congestion and temporary loss of network connectivity are handled without risking the patient's wellbeing. 
The objective of this thesis is to develop a generic framework for the architecture of an RPM system that is scalable, adaptable for different sensor technologies and medical conditions, non intrusive, real-time, mobile, cost effective and robust. The proposed RPM system architecture utilizes the higher bandwidth available with upcoming wireless technologies like $4 \mathrm{G}$ and beyond as well as fits in well with future trends in computing models like cloud computing.

The first part of this thesis proposes an Adaptive Information Content Management (AICM) methodology for the RPM system architecture that is robust and can handle short term network congestion as well as temporary loss of network connectivity without losing critical patient data. The proposed method ensures that the patient's welfare is not jeopardized due to network conditions. The thesis will demonstrate how the proposed AICM methodology for an RPM system can be applied to pressure sensitive mat sensor data to improve survivability of the RPM system under adverse network conditions. No clinical trial was done to collect pressure sensor data used in this research. The pressure sensor data used was collected during an earlier clinical trial by a separate research team at Carleton University.

An implementation of an RPM system using pressure mats to monitor bed occupancy of the patient will be presented to demonstrate how the proposed RPM system architecture can be functionally realized in practice. A usability trial will be conducted to gain understanding of the healthcare provider's requirement for the Graphical User Interface (GUI) with the RPM system. The implemented bed occupancy RPM system will be used for the usability trial. The results of the usability trial will be used to implement a GUI for the bed occupancy RPM system. 


\subsection{Thesis Contribution}

The following is a list of the research contributions that were made during this thesis research; the details of the contributions will be explained throughout the following chapters.

- Proposed a centralized processing architecture for an RPM system that will utilize the high bandwidth available with $4 \mathrm{G}$ and higher wireless network in the future. The proposed architecture can seamlessly integrate with future computing models like cloud computing. The proposed architecture is easy to maintain, upgrade and is scalable.

- An AICM method is proposed for the RPM systems that will significantly improve the survivability of the RPM system when there is network congestion causing a drop in available network bandwidth and/or temporary loss of network connectivity. The AICM method handles adverse network condition by reducing the bandwidth requirements of the RPM system while still preserving the critical patient data. The proposed AICM method is presented in Chapter 5.

- A buffering scheme for the AICM method is proposed to maximize the survivability time of the RPM system.

- The proposed AICM method was applied to the bed occupancy data collected in an earlier clinical trial to demonstrate how improved survivability for the RPM system can be realized. The proposed AICM method and buffering scheme presented in Chapter 5, 6 and 7 has been submitted for review in [4]:

V. Joshi, A. Arcelus, M. Holtzman, R. Goubran and F. Knoefel, "Adaptive Information Content Management and Buffer Caching Design for Robust 
Remote Patient Monitoring System," IEEE Trans. on Information Technology in BioMedicine, 2011 (under review).

- Implemented a bed occupancy RPM system using pressure sensitive mats to monitor bed entry/exit times of the patient. The implemented bed occupancy RPM system was tested in a lab and was trialed with a voluntary patient for one week. The system was also used to collect entry/exit data for the smart home project at Carleton University called Technology Assisted Friendly Environment for the Third Age (TAFETA) located at the Elisabeth Bruyere Hospital in Ottawa, Canada.

- A usability study was conducted by research teams at Carleton and Wilfrid Laurier University along with University of Toronto's Nursing school for the usability test and qualitative analysis of the GUI design of the RPM system which included the pressure sensitive mat sensor for the bed entry/exit monitoring along with a GPS sensor. A GUI prototype was developed for the usability study. Using the results of the usability study a GUI was designed for the bed occupancy RPM system. The proposed bed occupancy RPM system was presented at [5], [6] and [7]:

W. Sun, H. Nguyen, D.M. Doran, R. Goubran, S. Doherty, F. Knoefel, A. Tosine, V. Joshi, and E. Sadowski, "Integration of Bio-mobility Information with at Point-of-Care Decision Support System for Safer Client Care," presented at the Canadian Nursing Informatics Association, Mississauga, ON, Nov. 22-25, 2009.

W. Sun, H. Nguyen, D.M. Doran, R. Goubran, S. Doherty, F. Knoefel, A. Tosine, V. Joshi, and E. Sadowski, "Integration of Bio-mobility Information with at Point-of-Care Decision Support System for Safer Client Care," presented at the Sigma Theta Tau 23rd Annual Research Conference: 'Promoting Excellence in Nursing Practice Through Research'. London, ON, Apr. 30, 2010. 
H. Nguyen, D.M. Doran, R. Goubran, S. Doherty, F. Knoefel, A. Tosine, H. Nguyen, V. Joshi, W. Sun, and E. Sadowski, "Integration of Bio-mobility Information with at Point-of-Care Decision Support System for Safer Client Care," presented at the 4th National Community Health Nurses Conference, Toronto, ON, June 16-18, 2010.

The two posters presenting the user and sensor aspects of the RPM system was accepted and presented at the $e$-Health 2010, Vancouver, BC, May 2010, [8] and [9]:

D. Doran, R. Goubran, S. Doherty, F. Knoefel, A. Tosine, H. Nguyen, V. Joshi, W. Sun and E. Sadowski, "Technology Integration Into Home Care Nursing: Meeting the Challenges of Chronic Disease Management," poster presentation at e-Health 2010, Vancouver, BC, May 30 - June 3, 2010.

A. Tosine, H. Nguyen, V. Joshi, D. Doran, A. Parush, R. Goubran, S. Doherty and E. Sadowski, "User Research of a Proposed Home Care Clinician's Non-intrusive Remote Monitoring System's Graphical User Interface," poster presentation at e-Health 2010, Vancouver, BC, May 30 June 3, 2010.

\subsection{Thesis Outline}

Chapter 2 provides details of the current status of the RPM system. It also outlines objectives of the RPM system along with sensor technologies employed in the RPM system. It captures the various architectural design criteria for the RPM system.

Chapter 3 outlines the architecture of the existing RPM systems.

Chapter 4 proposes centralized data processing architecture for the RPM system that exploits the high bit rates available with current and future high capacity wireless network like $4 \mathrm{G}$ and beyond. It also provides a comparison of the proposed architecture with the architecture of the RPM systems currently being used. 
An AICM method for the RPM system is proposed in Chapter 5. This proposed method provides for a robust architecture for the RPM system with improved survivability in case of network congestion or temporary loss of network connectivity.

Chapter 6 proposes a data buffering scheme for the proposed AICM method to enhance the survivability of the RPM system.

Chapter 7 provides the details of the implemented bed occupancy bed entry/exit RPM system. It also gives details of the usability study for the GUI requirements for an RPM system. Implementation of a GUI for the bed occupancy RPM system based on the results of the usability study are also presented along with results of the patient trial conducted using the implemented bed occupancy RPM system. It then goes on to show an example of how the AICM method for the RPM system can be realized using the bed occupancy RPM system as an example.

Chapter 8 presents a discussion on different techniques that may be used for the AICM for an RPM system.

Chapter 9 presents conclusions from the thesis including the contribution and suggestions for future work.

References are listed at the end. 


\section{Chapter 2: Remote Patient Monitoring System Design Considerations}

\subsection{Background}

The WHO (World Health Organization) report "Preventing chronic diseases: a vital investment" estimates that without preventative actions 388 million people will die from chronic diseases in the next 10 years. Many of these deaths will occur prematurely, affecting families, communities and countries [10]. It also indicates that chronic disease does not only affect elderly people, "almost half of chronic disease deaths occur prematurely, in people under 70 years of age. One quarter of all chronic disease deaths occur in people under 60 years of age.", e.g. type- 2 diabetes in children and adolescence is becoming highly prevalent worldwide due to childhood obesity.

A 2007 study by independent economic think tank, The Milken Institute indicates that six chronic diseases like heart disease, hypertension, pulmonary disease, mental disorder, stroke and diabetes cost $\$ 180 \mathrm{~B}$ per year and indirect impact is close to $\$ 530 \mathrm{~B}$ in the USA [11].

The Center for Technology and Aging draft report shows that [2]:

- Eight out of ten older Americans are faced with the health challenges of one or more chronic diseases. 
- Chronic disease accounts for three-quarters of America's direct health expenditures.

- People with chronic disease cost 3.5 times as much to serve compared to others, and account for $80 \%$ of all hospital bed days and $96 \%$ of home care visits.

This has resulted in very one sided health care spending where $5 \%$ of patients use $50 \%$ of the health care budget. While the cost of caring and treating chronic diseases is increasing exponentially on the other hand there is an increasing shortage of healthcare providers in developed countries like the UK, USA and Canada [12], [13], [14], [15]. Ballooning healthcare cost is a major concern around the world and with the population aging at a faster rate the healthcare cost is set to rise at alarming rate.

An American study indicates that 17.6 percent of all Medicare hospital admissions are readmissions and a majority of these are avoidable and can provide a saving of up to $\$ 15$ billion annually [2]. Studies in Europe have shown similar savings [15], [16]. Monitoring patient mobility and balance can significantly reduce the number of falls in older adults. Falls are responsible for almost $43 \%$ of all unintentional injury deaths in older adults. Over 1.8 million seniors were treated in US hospital emergency departments for fall injuries, and one out of four was subsequently hospitalized [2]. Currently the Ontario government spends $42 \%$ of its budget on healthcare. The government of Ontario's 2010 report on "Ontario's Long-Term Report on the Economy" indicates that healthcare cost will grow to $50 \%$ of its budget in near future [17].

RPM systems will play a pivotal role in future healthcare model both as a mean to reduce cost as well as improving healthcare provided to the aging population. 


\subsection{RPM Systems in mHealth}

The increasing cost of medical care, increased bandwidth availability especially with wireless networks, reduction in cost of telecommunication, reliability of telecommunication network, low cost availability of high quality video equipment all have contributed to significant interest in use of mHealth for providing health care [18] [19][20].

The RPM system used in mHealth provides patient monitoring by transmitting sensor data from the patient site to the central node using end to end wireless technology [21], [22], [23], [24], [25]. The sensor data may be processed at the patient site or the raw data may be transmitted directly to the central node for further processing. The patient's mobile device provides continuous monitoring in real-time.

The mHealth market is growing and will see a significant spurt in next decade and RPM systems will share major portion of that growth. According to Juniper Research's Mobile Health Opportunities Report, "public and private healthcare providers will be able to save between $\$ 1.96$ billion and $\$ 5.83$ billion in healthcare costs through the use of remote patient monitoring using cellular networks by the year 2014" [25]. Currently heart rate monitoring constitutes the majority of the RPM systems currently in the market in the USA. The report predicts that "monitoring market, for chronic diseases such as Asthma, COPD and diabetes, could eventually represent a much larger market in terms of numbers of monitored individuals and even revenues" [25].

With high speed wireless network it is possible to explore the feasibility of RPM systems that will be completely wireless providing mobility, scalability and universal access. 


\subsection{Current Status of RPM System}

The RPM system operates autonomously with no patient or healthcare provider intervention except in the case of failure [15], [26], [27], [28], [29], [30], [31], [32], [33], [34], [35].

RPM can be used to:

- Improve quality of life of elderly as well chronically ill patients.

- Reduce the cost of healthcare services.

- Allow patients to chose where they want to live, specifically outside of supervised medical facilities since RPM systems can be operated anywhere.

- Improve quality of care by early detection of deteriorating medical condition resulting in prompt intervention, preventing the condition becoming critical, thereby reducing emergency visits and better treatment.

- It can act as a tool for preventative healthcare.

- It can also provide emergency alerts under emergency conditions.

- RPM systems can be tailored to provide personalized healthcare.

- RPM systems can improve post acute care management resulting in reduced readmission and better recovery.

Numerous RPM systems have been proposed using a variety of sensors, WSN technologies, network technologies to connect healthcare providers to patients with different of medical condition, e.g. cardiac monitoring [36], [37], [38], [39], [40], sleep [41], [42], [43], [44], [45], [46], [47], [48], [49], [50], snoring [51], mental health [52], fall and mobility detection [53], [54], [56], [57], [58], emergency audio help [59], [60], 
[61], respiratory [62], [63], blood pressure [64] and EEG monitoring [65], [66], [67]. This

list is not an exhaustive summary but does provide an overview of the some of the major areas of research in RPM.

RPM systems are available commercially to monitor different patient conditions some of them are listed in Table 2.1.

Table 2.1: Commercially available RPM Systems

\begin{tabular}{|c|c|c|}
\hline Company & Device & Vital Signs Monitored \\
\hline $\begin{array}{l}\text { Wave } \\
\text { Technology } \\
\text { Group [67] }\end{array}$ & $\begin{array}{l}\text { Wave EEG } \\
\text { Monitor }\end{array}$ & $\begin{array}{l}\text { EEG Monitor. } \\
\text { Connection via Bluetooth to Cell phone. }\end{array}$ \\
\hline Vitaphone [68] & Vitaphone 1300 & $\begin{array}{l}\text { ECG Recorder. } \\
\text { Connection via Bluetooth to Cell phone. }\end{array}$ \\
\hline Emfit [69] & $\begin{array}{l}\text { Non-Contact Vitals } \\
\text { Monitor and IP } \\
\text { Nurse-Call System }\end{array}$ & $\begin{array}{l}\text { Heart and Respiration rates and movement activity. } \\
\text { Connection via wireless to monitor and then } \\
\text { LAN/WLAN. }\end{array}$ \\
\hline Biotronik [70] & $\begin{array}{l}\text { CardioMessenger } \\
\text { I/II }\end{array}$ & $\begin{array}{l}\text { Cardiac Resynchronization Therapy Defibrillator } \\
\text { (CRT-D), Implantable Cardioverter Defibrillator } \\
\text { (ICD), Pacemakers. } \\
\text { Connection via wireless to monitor and then cellular } \\
\text { network. }\end{array}$ \\
\hline $\begin{array}{c}\text { Boston } \\
\text { Scientific }[71]\end{array}$ & $\begin{array}{c}\text { LATITUDE® } \\
\text { Patient Management } \\
\text { system }\end{array}$ & $\begin{array}{l}\text { CRT-D, ICD. } \\
\text { Connection via Bluetooth and Landline. }\end{array}$ \\
\hline Medtronic [72] & $\begin{array}{c}\text { CareLink Remote } \\
\text { Monitoring Network }\end{array}$ & $\begin{array}{l}\text { Insertable Cardiac Monitors, Pacemakers, CRT-D, } \\
\text { ICD. } \\
\text { Connection to wireless monitor and the cellular } \\
\text { network or landline connection. }\end{array}$ \\
\hline $\begin{array}{c}\text { St. Jude Medical } \\
{[73]}\end{array}$ & $\begin{array}{l}\text { Merlin@home } \\
\text { transmitter }\end{array}$ & $\begin{array}{l}\text { Pacemakers, CRT-D, ICD. } \\
\text { Connection to wireless monitor and then landline } \\
\text { connection. }\end{array}$ \\
\hline $\begin{array}{c}\text { TeleMedCare } \\
\text { Error! Reference } \\
\text { source not } \\
\text { found. }\end{array}$ & $\begin{array}{l}\text { TeleMedCare } \\
\text { Health Monitor }\end{array}$ & $\begin{array}{l}\text { Weigh, Body Temperature, Blood Pressure, Blood } \\
\text { Oximetry, Blood Glucose, Spirometry (lung function), } \\
\text { ECG (heart function). } \\
\text { Connection to cellular network or landline connection. }\end{array}$ \\
\hline
\end{tabular}


As seen from Table 2.1 the majority of the commercial systems RPM systems provide monitoring of the implanted cardio devices like pacemakers and defibrillators. RPM systems providing more comprehensive monitoring of variety of vital signs for chronic conditions are becoming available in the market e.g. TeleMedCare Health Monitor Error! Reference source not found.. Though capabilities of the commercially available RPM systems are currently limited more complex RPM system models will be coming to market as the demand grows.

\subsection{Objectives of RPM System}

Objectives of the RPM system that can monitor acute and chronic patient conditions are diverse and often conflicting. Based on the research in to RPM systems the most critical overall objectives of the RPM system are summarized below [2], [15], [26], [27], [28], [29], [30], [31], [32], [33], [34], [35].

\section{Chronic condition monitoring (non Real-Time critical)}

Chronic patients are monitored to detect changes/trends in the patient's medical condition over a period of time, generally on a daily/weekly basis. This allows for early detection of deteriorating medical conditions resulting in prompt intervention, reducing emergency visits and better treatment as well as monitoring patient response to a particular treatment. The monitored data is non real-time critical and is not used to generate any emergency alerts, e.g. sleep pattern, mobility/gait data, weight, cough, snoring etc.

\section{Acute condition monitoring: Emergency Alerts (Real-Time critical)}

If vital signs (biological as well as environmental) of the patient suddenly changes the healthcare provider must be notified immediately so timely assistance to the 
patient can be provided. This requires detection of sudden changes in the patient's signs (blood pressure, heart rate, fall, high temp etc) and/or environmental conditions (e.g. room temp, stove being on for too long etc) and notification to appropriate care provider without tangible delay.

\section{In Home and/or Outdoor Monitoring}

The RPM system should be capable of monitoring patients both inside their house as well as when they go outdoors. The sensors used for in house monitoring may be different than the ones used for monitoring when patient leaves the house. Wearable sensors are better suited for outdoor monitoring [75].

\section{Non-Intrusive sensors}

The sensors should not impede the patient's daily activities or in any way restrict their range of motion e.g. sensors used for sleep monitoring should not interfere with their sleep pattern. Sensors should not be obtrusive in any way that will make the patient feel self-conscious or inhibited either in private and/or public, this is even more important from a privacy perspective. Sensors also should not prove to be a health hazard, e.g. lose wires, electric shock due to water, snow etc. This is especially true of wearable sensors. Sensors used in the home or outside the home should not interfere with their social interaction or make other people feel constrained. Wearable sensors should not have dangling wires/components which can be health hazards.

\section{Unaided/Autonomous Monitoring}

Operation of the RPM system not only should be non-intrusive but it should be automatic and self-starting. Very minimal if any onus should be placed on patients 
in setup, operation and maintenance of the system. The operation of the RPM system needs to be transparent to the patient. Self-sustaining operation of the RPM system is critical if RPM systems are to achieve wide acceptance. The majority of the patients are elderly and with serious and chronic medical conditions and the RPM systems should not be an additional burden to them.

\section{24 Hour monitoring}

Many medical conditions e.g. fall, Electrocardiograph (ECG) etc, need to be monitored on a continuous basis. So the RPM system must be capable of monitoring patients around the clock 24 hours day.

\section{Monitoring of Biological and Environmental Signals}

Different patient conditions require a variety of biological and environmental signals to be monitored, e.g. blood pressure, ECG, room temperature, humidity, movement etc. Each patient will have different sensor needs. So the RPM system needs to be configurable to meet the requirement of individual patient monitoring needs.

\section{Patient Mobility}

The ability to monitor the patient over wide areas inside and outside home is important for ensuring patient mobility. The RPM system should not put a restriction on the patient's movements either inside or outside their residence. This means the system should be operable under any condition outside home e.g. different social settings, shopping mall, dinner with family/friends etc as well as in the home. If the RPM system limits patient mobility it will adversely affect patients buy in to the RPM system. 


\subsection{Signals Monitored by RPM Systems}

The RPM system is used to monitor both biological as well as environmental signals. Table 2.2 provides a list of biological signals typically monitored by RPM systems along with medical conditions for which they are used.

Table 2.3 provides a list of environmental signals typically monitored by RPM systems along with medical conditions and/or patient well being for which they are used [2], [76], [77]. Such environmental monitoring is especially helpful for patients with one or more cognitive disabilities. 
Table 2.2: List of Biological Signals commonly monitored using RPM systems.

\begin{tabular}{|c|c|}
\hline Biological Signal & Typical Medical Conditions \\
\hline Breathing Rate [62], [63] & $\begin{array}{l}\text { Cardiovascular, Pulmonary, } \\
\text { Psychiatric disorders, COPD }\end{array}$ \\
\hline $\begin{array}{l}\text { Heart Rate and Heart Rate Variability } \\
{[26],[38]}\end{array}$ & $\begin{array}{l}\text { Cardiovascular, Pulmonary, } \\
\text { Psychiatric disorders }\end{array}$ \\
\hline ECG [38] & $\begin{array}{l}\text { Cardiovascular, Pulmonary, } \\
\text { Psychiatric disorders }\end{array}$ \\
\hline $\begin{array}{l}\text { Electroencephalography (EEG) [65], } \\
{[66],[67]}\end{array}$ & $\begin{array}{l}\text { Seizure Disorders, Dementia, Head } \\
\text { Injuries, Brain Tumours, Infections, } \\
\text { Degenerative Diseases, and Metabolic } \\
\text { Disturbances that affect the brain }\end{array}$ \\
\hline $\begin{array}{l}\text { Saturation of Peripheral Oxygen (SpO2) } \\
{[26]}\end{array}$ & $\begin{array}{l}\text { Cardiovascular, Pulmonary, } \\
\text { Psychiatric disorders }\end{array}$ \\
\hline Body Temperature [26] & Fever \\
\hline Glucose [26] & $\begin{array}{l}\text { Diabetes, Vascular or Neurological } \\
\text { Complications }\end{array}$ \\
\hline Blood Pressure [26] & Hypertension \\
\hline Sleep [50] & $\begin{array}{l}\text { Sleep Disorders, Sleep Apnea, } \\
\text { Cardiovascular, Pulmonary, } \\
\text { Psychiatric disorders }\end{array}$ \\
\hline Mobility/Balance [53], [55] & $\begin{array}{l}\text { Pain, Arthritis, Post-Surgical } \\
\text { Recovery, Falls }\end{array}$ \\
\hline Weight [26] & $\begin{array}{l}\text { Cardiovascular, Pulmonary, Stress, } \\
\text { Psychiatric Disorders, Diabetes }\end{array}$ \\
\hline Audio [59], [60] & Seizure, Pulmonary, Sleep Apnea \\
\hline
\end{tabular}


Table 2.3: List of Environmental signals commonly monitored using RPM systems.

\begin{tabular}{|l|l|}
\hline \multicolumn{1}{|c|}{ Environmental Signals } & \multicolumn{1}{|c|}{ Typical Medical Condition } \\
\hline Room Temperature & Dementia, Psychiatric condition \\
\hline Humidity & Dementia, Psychiatric condition \\
\hline Fridge Door & Dementia, Psychiatric condition \\
\hline Prescription Medication & Dementia, Psychiatric condition \\
\hline Location & $\begin{array}{l}\text { Fall, Dementia, Psychiatric condition, } \\
\text { Heart Attack, Seizures }\end{array}$ \\
\hline Audio & Help Command, Audio recognition \\
\hline
\end{tabular}

\subsection{Remote Patient Monitoring System Components}

As shown in Figure 1.1 the main RPM system components include:

1. Sensors: Biological and environmental sensors are used to monitor different patient conditions. Sensors then transmit that data over a short distance $(1-10 \mathrm{~m})$ to a local node using low power wireless technology like Bluetooth, Zigbee etc [78]. Sensors may transmit raw data or can also have built in DSP processor to process the raw data before transmitting the processed signal. Sensors can be stationary or wearable and need to be non-intrusive.

Table 2.4 provides a list of typical sensors used in RPM systems along with the biological and environmental signals each sensor monitors and the typical data requirement for those signal data. It also gives which signal data may be used to provide emergency alerts. 
Table 2.4: Typical sensors currently used along with their data rate and real-time requirements.

\begin{tabular}{|c|c|c|c|c|}
\hline Sensor & $\begin{array}{l}\text { Condition } \\
\text { Monitored }\end{array}$ & $\begin{array}{l}\text { Monitoring } \\
\text { Frequency }\end{array}$ & $\begin{array}{c}\text { Transmission } \\
\text { Rate }\end{array}$ & $\begin{array}{l}\text { Real } \\
\text { Time } \\
\text { Alerts }\end{array}$ \\
\hline $\begin{array}{c}\text { Audio } \\
\text { (microphone) } \\
{[51]}\end{array}$ & $\begin{array}{c}\text { Cough, Wheezing, Sleep } \\
\text { Apnea, Audio command, } \\
\text { Seizure, Breathing Rate, } \\
\text { Heart Rate }\end{array}$ & Continuous & $\sim 350 \mathrm{kbps}$ & Yes \\
\hline IR Camera [57] & $\begin{array}{c}\text { Bed entry/exit, Balance, } \\
\text { Mobility. Breathing } \\
\text { Rate, Seizures }\end{array}$ & Continuous & $>1 \mathrm{Mbps}$ & No \\
\hline $\begin{array}{c}\text { Pressure Mats } \\
{[55]}\end{array}$ & $\begin{array}{c}\text { Bed entry/exit, Balance, } \\
\text { Mobility. Breathing } \\
\text { Rate, Sleep Apnea }\end{array}$ & Continuous & $\sim 150 \mathrm{kbps}$ & Yes \\
\hline $\begin{array}{c}\text { Video Camera } \\
\text { [79], [80] }\end{array}$ & $\begin{array}{c}\text { Bed entry/exit, Balance, } \\
\text { Mobility. Breathing } \\
\text { Rate, Seizures }\end{array}$ & Continuous & $>1 \mathrm{Mbps}$ & Yes \\
\hline $\begin{array}{l}\text { UWB Sensor } \\
{[81],[82]}\end{array}$ & $\begin{array}{c}\text { Bed entry/exit, Balance, } \\
\text { Mobility. Breathing } \\
\text { Rate, Seizures }\end{array}$ & Continuous & $>1 \mathrm{Mbps}$ & Yes \\
\hline $\begin{array}{c}\text { EEG [65], [66], } \\
{[67]} \\
\end{array}$ & Seizures, Dementia & Periodic & $>10 \mathrm{~K}$ bps & No \\
\hline ECG [36] & Heart Rate & Continuous & $\sim 20 \mathrm{Kbps}$ & Yes \\
\hline $\begin{array}{c}\text { Accelerometer } \\
{[44],[79]}\end{array}$ & $\begin{array}{c}\text { Balance, Mobility. } \\
\text { Breathing Rate, Sleep } \\
\text { Apnea, Heart Rate, } \\
\text { Exercise } \\
\end{array}$ & Continuous & $\sim 20 \mathrm{Kbps}$ & Yes \\
\hline GPS [2] & Location, Exercise, & Continuous & $\sim 10 \mathrm{Kbps}$ & Yes \\
\hline Weight & Weight & Periodic & 1 sample/day & No \\
\hline Glucose & Diabetes & Periodic & $\sim 5$ samples/day & Yes \\
\hline $\begin{array}{c}\text { Home Temp and } \\
\text { Humidity }\end{array}$ & Safety & Periodic & 1 sample/ $\min$ & Yes \\
\hline $\begin{array}{l}\text { Prescription } \\
\text { Medication } \\
\end{array}$ & Compliance & Periodic & $\sim 5$ samples/day & No \\
\hline
\end{tabular}


2. Local Node: The local node connects to all the sensors and receives data from them. The local node can perform different operations on the received data, e.g. data can be processed further to obtain signal features, and it may store the data for store-and-forward mode method. It also provides connectivity for sending raw or processed data over the Internet to the central node. The local node may also provide the interface for the patient to view their monitored data. The local node can be a PC, mobile phone or a dedicated device depending on the functionality it provides.

3. Central Node: The central node receives raw or processed data from all the patients connected to RPM system. It acts as a repository for all the patient data. It may also have connectivity to the patient's medical records using the Electronic Health Record (EHR) system. The central node may need to further process and analyze the patient data and may evaluate patient status, trends in the patient's medical condition and may generate emergency alerts if the patient's condition is critical. For emergency alert capability data must be processed in real-time.

Signal/data processing is shared between the local and the central node. How the processing load is distributed across the central and the local node is an RPM architectural design consideration.

It also provides storage for the RPM patient data it receives. The central node also acts as an access node for healthcare providers to access the monitored and analyzed patient data to evaluate patient condition, treatment progress, any early signs of deteriorating condition etc. The access maybe provided using a Web based GUI. 
4. Network Connectivity: Connectivity between the local and the central node can be provided using wired or wireless technology. A local server acts as a bridge between its sensors and a central server, which may also serve as a gateway to a Wide Area Network (WAN) such as the Internet or a mobile Wireless WAN (WWAN). As mentioned in Chapter 1, newer generations of wireless broadband technologies like $3 \mathrm{G} / 4 \mathrm{G}$ do offer data rate in excess of $100 \mathrm{Mbps}$. While the penetration and reliability of these newer wireless technologies is still low, high capacity wireless technologies will become widely available in the near future [3], [21].While it is possible to use existing wired broadband networks while patient is home, only wireless network can be used when patient is outside the home.

\subsection{RPM System Architecture Design Criteria}

The RPM system architecture needs to meet the objectives of the RPM system defined in Section 2.1. The critical architectural design criteria for the RPM system can be summarized as shown in Table 2.5. The following subsections discuss each criterion in detail.

Table 2.5: Design Criterion for RPM system.

\begin{tabular}{|c|}
\hline RPM System Architectural Criteria \\
\hline Cost \\
\hline Scalability \\
\hline Network BW and Data Compression \\
\hline Network QoS and Reliability \\
\hline Wireless Technologies \\
\hline Data Security \\
\hline Real-time signal processing \\
\hline Sensor Battery Life \\
\hline
\end{tabular}




\subsubsection{Cost}

The cost of the RPM system includes capital, installation and operational cost as listed below:

1. Equipment Cost: This includes cost of all the RPM system components like Sensors, Routers, Server, Database, Wireless device (PDA etc). This cost will reduce significantly with wider deployment and high volume. Using standard offthe-shelf components will reduce the hardware cost, development cost and cost of maintenance.

2. Installation Cost: This is the cost of initial installation of the RPM system at the patient's home and the cost of the central node installation.

3. Network Cost: The sensor data sent over the network by the RPM system can be very high, especially when high data rate sensors like audio and video are used. The data rate can be as high as $1 \mathrm{Mbps}$ or more in such cases, especially when monitoring is continuous. Cost of transmitting monitored data to the central node in such case can be high, e.g. a 1 Mbps system could be transmitting $\sim 90 \mathrm{~Gb}$ of data per day. The cost of such high data transmission over current networks can be prohibitive for RPM system. Unless the transmission cost per bit is reduced from current levels different techniques to reduce the data sent by high data rate sensor must be considered in the RPM system design to make it affordable.

4. Maintenance Cost: The cost of maintaining the RPM system can be significant especially given the high reliability requirements of the RPM system. RPM system architecture can have significant influence on the maintenance cost. The cost of maintaining system components at remote patient site will be the major 
factor influencing overall maintenance cost of the RPM system. With a large number of patient sites spread over a wide geographical area, the cost of maintaining local nodes will be significant. The number of components at patient the site will significantly affect the RPM system cost, the more the components at patient site, more maintenance will be required. Using off-the-shelf components instead of proprietary hardware and software will also reduce maintenance costs since they are easier to obtain and generally cheaper.

5. System Upgrade Cost: The RPM system hardware (including sensors) and software will be upgraded over a period of time either to replace existing hardware or software with newer versions. The upgrade process needs to be nonintrusive and should not affect system reliability. The upgrade procedures that require access to the patient's home need to be kept to a minimum since access to patient homes is costly, intrusive and will affect the patient's daily activity and can affect patient acceptance of the RPM system. The cost of upgrading the RPM system can get significant when large number of RPM systems is in operations.

Lack of standardization and use of proprietary technologies for most of the RPM system components (sensors, WSN, EHR, data security and GUI etc) has lead to a silo effect with different RPM systems using incompatible interfaces. This leads to higher initial investment and maintenance cost. Adding new services or devices to existing systems can be a challenge requiring custom modification, high development cost and higher maintenance cost. Use of standardized rather than proprietary technologies will make RPM systems cost-effective and will help achieve higher penetration. 
Continua Health Alliance, a non-profit, open industry coalition of the healthcare and technology companies, is driving the standardization of the RPM system technologies [83]. Currently it has more than 200 members across the world. The objective of the alliance is "to improve the quality of personal healthcare. Continua is dedicated to establishing a system of interoperable personal health solutions with the knowledge that extending those solutions into the home fosters independence, empowers individuals and provides the opportunity for truly personalized health and wellness management.” [83]

\subsubsection{Scalability}

The RPM system needs to be scalable since each patient may need different medical conditions to be monitored. Scalability allows tailoring of each RPM system for each individual patient as well as provide the ability to add more sensors if needed. New sensors are being constantly introduced in the market. These sensors may replace existing sensors or provide the ability to monitor additional biological/physiological signals.

The RPM system should be able to add/delete sensors from the local sensor network with minimal manual intervention. The sensor network datafill should handle addition/deletion of sensors automatically. So the local sensor network for the RPM system should be auto-configurable.

As penetration of RPM systems in healthcare industry grows the number of patients connected to the central node will increase significantly. Also with the increase in number of signals being monitored the data per patient will also grow. The central node acts as interface between the patient, the healthcare professional, patient records and a clinical decision support system if available. It is a central repository of all the monitored patient data and must provide a seamless interface to healthcare providers on a real time basis. 
The database access for patient information must be fast. So the central node must be scalable to handle an increased dataflow as well as increased access by users in a seamless manner. Constant interruption to upgrade or poor performance will not be accepted by users and can lead to loss of critical patient data. The central node should be designed so that it is not a bottleneck limiting system performance.

The increase in patients and signals being monitored will affect the profile of the healthcare providers using the RPM system. Signals being monitored can change the type of healthcare providers accessing the data. In some cases specialist from different hospitals may collaborate for treatment in which case an enterprise solution will be needed for the central node. This will add to the security and confidentiality requirement for the node. The scalable design of the central node will become essential to handle the future growth. Scalability of the RPM system is an important design criterion.

\subsubsection{Data Compression}

Power consumption of battery operated sensor must be kept low to prolong the battery life. The majority of the battery power consumption happens when the sensor is transmitting data so keeping the sensor data to a minimum will prolong the battery life. Data compression in the RPM system may be used to reduce data transmitted by the sensor to the local node. Data compression techniques could be lossless or lossy [78].

While lossless compression is ideal since no information is lost, depending on the signal being monitored it may not offer significant reduction in data rate. Lossy compression can provide much higher compression ratio but at reduced quality since information content is reduced by lossy compression. So a trade-off exists between the sensor data compression algorithm used and the battery life. The choice of data 
compression algorithm will also be dictated by the kind of data the sensor is transmitting and criticality of the data.

\subsubsection{Network QoS and Reliability}

To prevent loss of patient data the communication networks must meet bandwidth requirement of the RPM system [38], [84]. Real-time critical patient data requires that network connectivity between sensors, the local node and the central node is constantly maintained. This makes reliability of underlying communication network used by RPM system a must.

Providing support for real-time RPM application over IP networks imposes new requirements for IP network architecture. The IP network is a packet switched network and was designed with data services like Internet, IP VPNs etc in mind. The service model for IP network is the "Best Effort" model. The best effort model does not guarantee packet delivery. Since IP protocol does not guarantee delivery many applications requiring guaranteed delivery use TCP protocol on top of IP to ensure packet delivery [85], [86], [87], [88], [89].

Packet loss happens in IP networks due to congestion. Congestion can be due to demand being higher than the capacity of the network, sudden increase in traffic, changes in routes, links going down etc. Congestion in IP network results in:

- Delay: Packets get queued up at the router/server, taking longer time to exit the router introducing delay in packet arrival time at the destination.

- Packet Loss: The queue buffers in routers/servers get exhausted, routers/servers start dropping packets resulting in loss of transmitted data. 
- Jitter: Packets sent from the source follow different routing path having variable delay so packets are received at the destination with varying length of delay time.

Sensor data in the case of RPM systems is generally non-bursty. The delay, jitter and loss of data due to communication channel will affect reliability of the RPM system and in the case of critical patient data may put the patient's life in danger [38]. The IP network used by the RPM system needs to provide the following services:

- Adequate bandwidth provision (to avoid general congestion)

- Rapid traffic restoration (to keep disruption after a failure to acceptable levels)

- Admission control (to avoid local congestion affecting individual user sessions)

Network QoS is a broad term used to describe how different application traffic is treated by the communication network. The QoS allows the network to satisfy specific service requirements of a particular application over all the underlying networks like ATM, Frame Relay, Ethernet and 802.1 networks, SONET, and IP-routed networks. The QoS provides better service by guaranteeing bandwidth, managing congestion, set priority for traffic etc.

For the real-time requirements of emergency response, the reliability needs for the patient data delivery and need for patient mobility requires QoS guaranteed from the communication network being used by the RPM system. The network needs to have the ability for the QoS provisioning for the RPM system [89], [90].

The network can provide three basic levels of end-to-end QoS [85], [86], [87], [88]:

- Best-effort service: Does not guarantee packet delivery.

- Differentiated service (soft QoS): Traffic is differentiated in to different classes and is handled based on the QoS mechanism defined for that class. Some classes 
are treated better than the others (faster response, more average bandwidth, and lower average loss rate). This differentiated treatment is not a hard guarantee over the end-to-end connection as each service provider may interpret the QoS differently. This is especially true if the connection involves multiple hops.

- Guaranteed service (hard QoS): This service requires reservation of network resources so the network can meet the traffic flow's specified service requirements. This service is provided using Resource ReSerVation Protocol (RSVP). Guaranteed service requires prior network resource reservation over the connection path. It is hard to reserve network resources over the Internet.

Network QoS for real-time applications is implemented using following mechanisms in routers [85], [86], [87], [88]:

- Classifier: The packets are classified based on some filtering criteria specified by the originator.

- Marker: Packets are marked to indicate the class of service to which they belong. The marking is done using DiffServ/TOS (Differentiated Services/Type of Service, IPv4) or Traffic Class Octet (IPv6). This field indicates to network how each packet should be treated.

- Shaper: Provides a smoothing effect to bursty traffic using buffering. For realtime applications shaper should not be used.

- Scheduler: Scheduler decides how the packets are queued out. Two types of schedulers are used namely, priority schedule and weighted scheduler. Priority scheduling provides the least amount of delay. 
- Queue Management: Queue management decides how the packets are handled when queue buffer gets full. Tail drop management just drops packet when the queue becomes full. Active Queue Management (AQM) drops packets when a certain queue buffer depths are exceeded. Real-Time applications should not use AQM.

As seen from the multiple mechanisms for the QoS mentioned above there are different ways to control delay, loss and jitter in the network. The QoS mechanism needs to be tailored for each real-time application.

There are different technologies used for switching signals in high speed networks like Ethernet, ATM (Asynchronous Transfer Mode) and MPLS (Multiprotocol Label Switching). Each of these technologies provides support for the QoS.

- Ethernet IEEE 802.1Q: Most of the RPM systems will use LAN using Ethernet so the QoS over Ethernet is critical for real-time applications. Ethernet IEEE 802.1Q provides 4 bytes for the QoS mechanism. The VLAN id field is used to group certain type of traffic based on common requirements and handled based on different QoS requirements e.g. based on DiffServ. It also has 3 bits to define 8 classes of services [90].

- ATM: ATM is a virtual connection oriented system with each connection setup associated with its QoS requirements, e.g. delay, loss and cell delay. With highly guaranteed QoS, the ATM network is highly suitable for real-time applications. ATM provides four classes of service: Constant Bit Rate (CBR), Variable Bit Rate (VBR), Available Bit Rate (ABR) and Unspecified Bit Rate (UBR). Due to the fixed size of ATM cells jitter and delay in ATM network is minimized [87], [88]. 
- MPLS: MPLS networks operate over existing IP network but uses underlying IP network facilities. One advantage of the MPLS network is its ability to recover from failures quickly and also have backup paths available in case a failure occurs. Though MPLS does not provide QoS mechanism it can be used with DiffServ or RSVP protocol to provide the desired QoS. The Virtual Circuits (VCs) of a the Label Switched Paths (LSPs) of MPLS, can be dedicated to the use of specified traffic types and can be integrated with traffic differentiation schemes such as DiffServ [85].

One way to ensure the network is connected is for the local node to send a regular heartbeat signal to the central node. The lack of regular heartbeat from the local node may be used to generate an alert to healthcare providers to verify patient and network status.

Use of the RPM system for real-time and critical patient data requires that a mechanism to ensure that network QoS meets the RPM system performance requirement is mandatory.

\subsubsection{Wireless Technologies}

\subsubsection{Wireless Sensor Network Technologies for RPM}

The WSN is an essential part of the RPM system for unobtrusive monitoring of patients as well as providing patient mobility [32], [78], [91], [92], [93]. Unobtrusiveness of the RPM system will significantly help with patient acceptance and reducing constraints put on patients.

Wired sensors are expensive to install and scalability of a wired network is limited. On the other hand wireless sensors can be installed easily and cheaply. Another 
advantage of wireless sensors is that they can be deployed in large quantities. WSN is very easy to scale up or down.

The main wireless protocol technologies used or proposed for the RPM system WSN are Bluetooth, Bluetooth Low Energy (LE), Zigbee and UWB [94], [95], [96], [97].

1. Bluetooth: Bluetooth protocol is a short range $(\sim 10 \mathrm{~m})$ Wireless Personal Area Network (WPAN) technology defined by Bluetooth SIG [98]. It is geared towards voice and data application. IEEE 802.15.1-2005 protocol was derived from Bluetooth V1.2 protocol. While Bluetooth protocol offers data transfer rates of up to $1 \mathrm{Mbps}$ its high battery power consumption and low number of nodes (7) poses major constrains for its adoption to an RPM system sensor network [98].

2. Bluetooth Low Energy (LE): In 2006 Nokia along with its development partners released low power usage protocol based on Bluetooth technology under brand name "Wibree". In 2007 "Wibree" was adopted as Bluetooth Low Energy technology. In July 2010, the Bluetooth SIG announced the formal adoption of Bluetooth Core Specification Version 4.0 with the hallmark feature, low energy technology [98].

Bluetooth LE can provide battery life of up to 1 year and can support unlimited number of nodes in the network (implementation dependent) with a data transfer rate of up to $1 \mathrm{Mbps}$. Low power consumption allows Bluetooth LE based sensors the use of button/coin cells and other energy saving solutions [98].

3. ZigBee: The ZigBee is another protocol developed for WPAN applications. It is developed on top of the IEEE 802.15.4 protocol standard. The standard is maintained by the ZigBee Alliance which consists of a large number of 
companies [99]. ZigBee supports mesh networking topology. It offers lower power consumption and is a low cost alternative to Bluetooth and Bluetooth LE. ZigBee devices can transfer data at a rate of up to $250 \mathrm{Kbps}$. Since ZigBee technology can support a large number of nodes its low power consumption makes it a viable alternative for the RPM system sensor networks.

4. Ultra Wide Band (UWB): UWB technology based on impulse radar (IR) has attracted significant attention for the RPM system sensor networks due to its high data transfer rate (up to $110 \mathrm{Mbps}$ ) due to its low cost and minimal EMI interference to other radio and medical equipment. UWB technology proposed for the RPM system is based on IEEE 802.15.4a standard approved in March 2007. IEEE 802.15.4a standard defines "Wireless Medium Access Control (MAC) and Physical Layer (PHY) Specifications for Low-Rate Wireless Personal Area Networks WPANs): Alternate PHYs" [100]. UWB technology based on IEEE 802.15.4a offers high data rates from $850 \mathrm{kBps}$ to $20 \mathrm{Mbps}$ and can be used for simultaneous monitoring of many continuous physiological signals. The disadvantage of higher data rate operation is that battery life is very short. So even though the UWB system does not use any more power than ZigBee ( $8 \mathrm{~mJ} /$ Mbytes), higher data rates come at the cost of battery life. UWB technology is not mature; the FCC approved its use in 2002, but does show significant potential for higher data rate application for the RPM systems [101], [102], [103], [104].

IEEE $802.11 \mathrm{a} / \mathrm{b} / \mathrm{g}$ is a standard that is defined for wireless local area networking (WLAN). It offers data transfer rate from $1 \mathrm{Mbps}$ to over $50 \mathrm{Mbps}$. The maximum 
transmission range is $\sim 100 \mathrm{~m}$. While wireless sensor networks based on IEEE $802.11 \mathrm{a} / \mathrm{b} / \mathrm{g}$ (e.g. Wi-Fi) does offer high transfer rates and wide ranges the high power consumption limits its use in a mobile RPM system at present time.

Table 2.6 provides a summary of the main characteristics of these protocols relevant to the RPM system. Figure 2.1 shows the data rate and range for various a wireless technologies.

Table 2.6: Wireless Sensor Technology Protocols.

\begin{tabular}{|l|c|c|c|c|}
\hline \multicolumn{1}{|c|}{ Standard } & Bluetooth & $\begin{array}{c}\text { Bluetooth } \\
\text { Low Energy }\end{array}$ & Zigbee & UWB \\
\hline IEEE Specification & 802.15 .1 & 802.15 .1 & 802.15 .4 & $802.15 .4 \mathrm{a}$ \\
\hline Frequency Band & $2.4 \mathrm{GHz}$ & $2.4 \mathrm{GHz}$ & $\begin{array}{c}868 / 915 \mathrm{MHz} ; \\
2.4 \mathrm{GHz}\end{array}$ & $3.1-10.6 \mathrm{GHz}$ \\
\hline $\begin{array}{l}\text { Maximum Signal } \\
\text { Rate }\end{array}$ & $1-3 \mathrm{Mb} / \mathrm{s}$ & $1 \mathrm{Mb} / \mathrm{s}$ & $250 \mathrm{~Kb} / \mathrm{s}$ & $\begin{array}{c}1 \mathrm{Mb} / \mathrm{s} \\
\text { mandatory, up } \\
\text { to 27 Mb/s }\end{array}$ \\
\hline Range & $10 \mathrm{~m}$ & $10 \mathrm{~m}$ & $10-100 \mathrm{~m}$ & $10 \mathrm{~m}$ \\
\hline $\begin{array}{l}\text { Number of RF } \\
\text { Channels }\end{array}$ & 79 & 40 & $1 / 10 ; 16$ & $1-5$ \\
\hline Network Topology & Star & Star & Star/Mesh & Distributed \\
\hline Battery Life & $5-10$ Days & 1 Year & $1000+$ Days & $\sim 1$ Day \\
\hline $\begin{array}{l}\text { Maximum Number } \\
\text { Cell Nodes }\end{array}$ & 8 & unlimited & $>65000$ & 8 \\
\hline
\end{tabular}




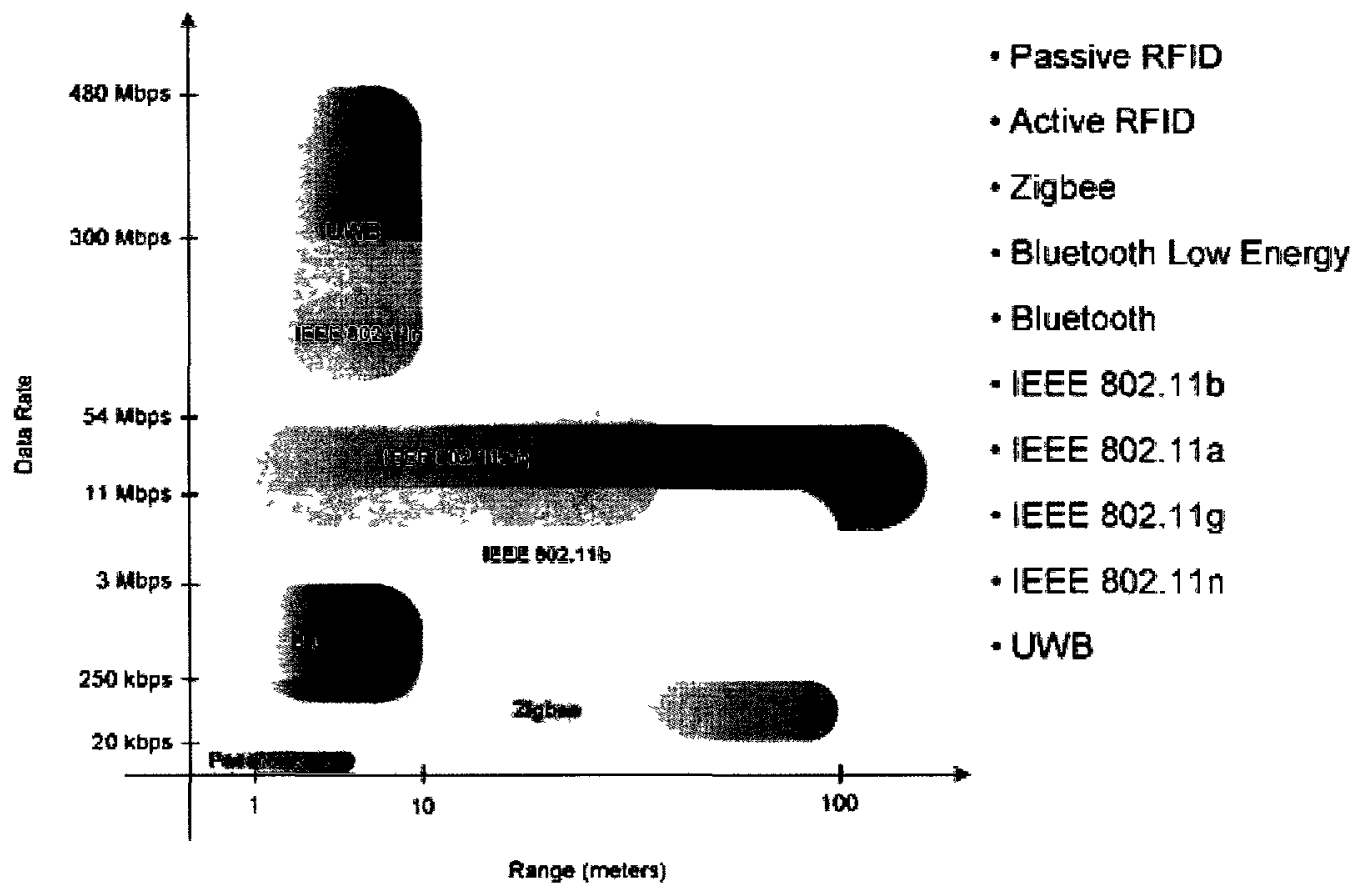

Figure 2.1: Power consumption, data rate and range are the three key considerations when selecting a wireless interface reproduced from: [96], [97].

All battery operated wireless sensors using the technology discussed above have a small communication range (approx. 10m). Due to limited range of wireless sensors the wireless sensor network for the RPM system at patient home will consist of a large number of sensors and relay nodes, constituting a multi-hop network. Such a network of sensor nodes is highly scalable and allows for wide coverage area without limiting patient mobility. The network can have redundancy built in to it for increasing fault tolerance.

In 2009 Continua Health Alliance selected Bluetooth LE and ZigBee technology for the RPM and telemedicine applications [83].

The IEEE 802.15 Task Group (TG) 6 Body Area Network (BAN) is developing a communication standard optimized for low power devices and operation on, in or around the human body (but not limited to humans) to serve a variety of applications including medical, consumer electronics / personal entertainment [105]. The WBAN channel 
models defined by IEEE TG6 cover both implant and on-body scenarios. Implanted devices are operating within the frequency range of $402-405 \mathrm{MHz}$, whereas communication in other frequency bands is on-body including $2.4 \mathrm{GHz}$ ISM and $3.1-$ 10.6 GHz UWB bands.

\subsubsection{Wireless Broadband Technology for RPM}

Mobile cellular technologies (GSM, GPRS, CDMA2000, and WCDMA, 3/4G, LTE etc) are needed to connect the local node (which can be mobile or fixed depending on the architecture) to the central node over WANs. High speed wireless networks are one of the main enabler for RPM systems becoming widely available and deployable.

4G wireless networks offers significantly higher data rates compared to current $2 \mathrm{G}$ and $3 \mathrm{G}$ technologies. Table 2.7 shows various wireless network technologies currently being deployed and their data rates. Sprint, Verizon and AT\&T have started rolling out their individual $4 \mathrm{G}$ wireless networks in the USA and roll out is expected to accelerate next year [106], [107]. The majority of the American cable provider's offer 3-15 Mbps download broadband service, higher-speed broadband is being planned [108]. 
Table 2.7: Maximum data rate available over different wireless networks.

\begin{tabular}{|c|c|c|c|}
\hline \multirow{2}{*}{\multicolumn{2}{|c|}{$\begin{array}{l}\text { Wireless Network } \\
\text { Technology }\end{array}$}} & \multicolumn{2}{|c|}{ Data Rate } \\
\hline & & Download & Upload \\
\hline \multicolumn{2}{|c|}{ 2.5G GPRS } & $114 \mathrm{Kbps}$ & $20 \mathrm{Kbps}$ \\
\hline \multicolumn{2}{|c|}{ 2.75G Edge } & $384 \mathrm{Kbps}$ & $60 \mathrm{Kbps}$ \\
\hline \multirow{5}{*}{$3 \mathrm{G}$} & UMTS & $384 \mathrm{Kbps}$ & $64 \mathrm{Kbps}$ \\
\hline & W-CDMA & $2 \mathrm{Mbps}$ & $153 \mathrm{Kbps}$ \\
\hline & EV-Do Rev A & 3.1 Mbps & $1.8 \mathrm{Mbps}$ \\
\hline & HSPA 3.6 & 3.6 Mbps & $348 \mathrm{Kbps}$ \\
\hline & HSPA 7.2 & 7.2 Mbps & $2 \mathrm{Mbps}$ \\
\hline \multirow{4}{*}{ Pre 4G } & WiMax & $100 \mathrm{Mbps}^{+}$ & $56 \mathrm{Mbps}$ \\
\hline & LTE & $100 \mathrm{Mbps}+$ & $50 \mathrm{Mbps}$ \\
\hline & HSPA+ & $56 \mathrm{Mbps}$ & $22 \mathrm{Mbps}$ \\
\hline & HSPA 14 & $14 \mathrm{Mbps}$ & 5.7 Mbps \\
\hline \multirow{2}{*}{$4 \mathrm{G}$} & $\begin{array}{r}\text { WiMax } 2 \\
(802.16 \mathrm{~m})\end{array}$ & $\begin{array}{l}100 \text { Mbps Mobile } \\
1 \text { Gbps fixed }\end{array}$ & $60 \mathrm{Mbps}$ \\
\hline & LTE Advanced & $\begin{array}{l}100 \mathrm{Mbps} \text { Mobile } \\
1 \text { Gbps fixed }\end{array}$ & $?$ \\
\hline
\end{tabular}

$4 \mathrm{G}$ wireless broadband will expand the scope of available RPM services, but the $4 \mathrm{G}$ technology is in a preliminary stage of deployment and penetration is still limited. Also newer applications utilizing $4 \mathrm{G}$ data rate will introduce network congestion and the QoS may suffer.

\subsubsection{Data Security and Privacy}

Security and privacy of the medical information exchanged by the RPM system present a major hurdle for the use of the RPM systems. Since the RPM system will be transmitting 
the patient's confidential medical information over the network, on both LAN and WAN, security and privacy concerns must be addressed by any prospective RPM system design. Patient data being monitored is confidential and potential for unscrupulous and/or unethical employers, insurance companies, media and malicious hackers to obtain illegal access exists. Hence data security is a critical component of the RPM system. The RPM system must comply with the patient data privacy regulations. The RPM systems acceptance will depend on its ability to protect the privacy and confidentiality of the patient data [112], [113], [114], [115].

Privacy laws and regulations across Canada, include the concepts of accountability, specified purposes, consent, data minimization, limited collection, use, retention and disclosure, and safeguards. In Ontario, the Personal Health Information Protection Act, $2004(P H I P A)$ "creates rules for the collection, use and sharing of personal health information by "health information custodians", such as doctors, pharmacists and other health care providers. PHIPA also gives patients rights around access to their health records and how their health information is used" [116]. As mentioned in the report by Information and Privacy Commissioner (IPC) of Ontario "privacy subsumes a set of protections far greater than security. Although building strong security features into a technology is vital to preventing privacy breaches, technological security is only one of the means used to achieve informational privacy" [117]. "Under PHIPA, the Information and Privacy Commissioner's Office of Ontario, Canada (IPC) has issued Health Orders that provide guidance to the health care sector on the use of wireless communication technologies and mobile computing devices" [117]. 
In the USA the transmission and storage of remotely monitored patient data must comply with the Health Insurance Portability and Accountability Act of 1996 (HIPAA) regulations [118]. The HIPAA regulations provide the rules for security and privacy of patient data. The HIPAA Privacy Rule, "which protects the privacy of individually identifiable health information; the HIPAA Security Rule, which sets national standards for the security of electronic protected health information; and the confidentiality provisions of the Patient Safety Rule, which protect identifiable information being used to analyze patient safety events and improve patient safety" [118].

RPM systems are susceptible to passive eavesdropping, packet injection and are vulnerable to many other security attacks. Different security threats posed to privacy of RPM data can be broadly classified under Passive and Active categories [112], [114], [115].

- Passive Threats: The monitored and transmitted data can be intercepted by attackers (eavesdropping). Such intercepted data can provide significant information about the patient's medical condition, physical status, location, activities, personal lifestyle and habits.

- Active Threats: Active threats include altering monitored and transmitted data and Denial of Service (DoS) where jamming can make the system unusable. This can result in false reading of medical condition, false alarm generation or suppression. This poses a serious risk to the patient's life.

The RPM system can protect patient information by using authentication and encryption.

Authentication: An authentication protocol is a must to ensure that data is coming from an authorised person/device. The authentication protocol may include password, 
digital signature using public key challenge-response authentication protocol or biometrics.

Encryption: Encryption of the data being transmitted can prevent unauthorised users from eavesdropping. Encryption can be accomplished in hardware as well as in software, e.g. availability of public key cryptography in the local and the central node will allow for data encryption. Both hardware and software encryption methods should be used to ensure the highest level of security.

There are many network communication cryptographic protocols like Transport Layer Security (TSL) or its predecessor Secure Socket Layer (SSL) and Internet Protocol Security (IPSec) which can be used to ensure that patient data is transmitted securely over the Internet [113].

While cryptographic protocols mentioned above are used for communication over the Internet they have significant overhead which may restrict their use in sensor network due to battery power and range limitations of the sensors. An alternative is to use a protocol like TinySec. TinySec is a low overhead link layer security protocol specifically designed for sensor networks. It offers two options 1) Authentication Encryption (TinySec-AE) and 2) Authentication Only (TinySec-AO). The increase in energy consumption due to $\mathrm{AE}$ is $10 \%$ while due to $\mathrm{AO}$ is $3 \%$ [113].

Another alternative is to use hardware that provides data encryption, e.g. CC2420 and CC2530 from TI which are a single-chip $2.4 \mathrm{GHz}$ IEEE 802.15.4/Zigbee compliant RF transceiver providing Hardware MAC encryption [119].

With higher penetration of RPM systems and privacy legislation it is expected that wider hardware and software security support will become available in future. Balancing 
the design of the security protocols with low power consumption and latency requirements is a major challenge.

\subsubsection{Real-Time Signal Processing}

Many of the monitored biological and/or environmental signals, e.g. Temperature, ECG, breathing/heart rate, fall detection, etc, may generate emergency alerts. For such signals end to end delay is critical. End to end delay includes signal processing and network transmission latency. The signal processing and network latency must meet the real-time criterion for such signals so that emergency alerts can be generated in a timely manner. Also real-time critical data requires that network connectivity between sensors, the local node and the central node is constantly maintained. This makes reliability of the underlying communication network an important requirement. While monitoring acute conditions it is critical that emergency alerts are generated in real-time so that healthcare providers can provide prompt response.

\subsubsection{Sensor Battery Life}

Patient mobility is a critical need for the RPM system. So a majority of the sensors used for the patient monitoring need to be battery operated. Battery operated sensors provide mobility as well as the ability to monitor the patient inside and outside the home. Constant monitoring of the patient using wireless sensor makes long battery life very important. Short battery life will put significant onus on patients to monitor and replace batteries frequently. This is especially onerous for elderly patients and will lead to low acceptance of the RPM system [75], [78], [120], [121].

The major power drain on the sensor power supply occurs when the sensor is transmitting data. So transmission mode, periodic or continuous, as well as data 
transmission rate will determine sensor battery life. Table 2.6 shows the battery life for different wireless sensor technologies [75], [96], [121].

As seen from Table 2.6 Zigbee and Bluetooth LE protocols provide significantly longer battery life compared to Bluetooth/UWB/Wi-Fi. But the data rate for Bluetooth LE and Zigbee is significantly lower than UWB and Wi-Fi. Bluetooth LE and Zigbee can also connect a large number of nodes to the network. One of the advantages of low battery power consumption is that it allows use of smaller form factor batteries for Bluetooth LE and Zigbee.

The sensor battery power consumption will have significant impact on the choice of wireless sensor technology used in the RPM system. Since the data transfer rate significantly affects the sensor battery life the RPM system architecture may embed some signal processing function in the sensor itself so as to reduce data transmitted over WSN. This can help prolong battery life.

Newer power scavenging/harvesting techniques are being researched to prolong battery life for wireless sensors [75], [121].

\subsection{Summary}

This chapter summarizes the drivers for RPM systems, and the objectives that the RPM system must satisfy for it to achieve wider penetration. It also describes the technologies used as building blocks of the RPM system along with design considerations involved with the RPM system.

The percentage of older adults in the general population is rapidly increasing especially in developed countries. They also constitute major portion of demographic with chronic illnesses. Given the high life expectancy in the current generation, a significant 
proportion of medical care expenses will be spent on providing chronic care. This has prompted very high interest in research in to the RPM systems. The RPM systems have been shown to benefit both patients and healthcare providers. The RPM systems improve quality of life for the patients by early intervention/detection before their problem becomes an emergency. By reducing the number of visits to emergency wards and early detection it also helps reduce the cost for the health care providers.

An RPM system for the patient care must meet many complex and conflicting objectives, e.g. monitoring the patient continuously without hindering their mobility and with the ability to provide emergency alerts in real-time and still providing privacy and maintaining confidentiality of the patient data.

While there is extensive ongoing research in to a variety of sensors like EEG, microphone, IR Video etc for RPM, most of the work is still focused on individual sensor application rather than the design of a comprehensive RPM system scalable for use with multiple sensor setups. Many issues related to RPM system design, e.g. cost, network QoS, data security, battery life still need to be fully examined. 


\section{Chapter 3: Current RPM System Architecture}

The RPM system objectives as defined in section 3.1 are quite diverse. End to end data transfer rates between a local node at a patient's home and the central node at the healthcare site is one of the major factors in the design of a wireless RPM system. Table 3.1 shows some of the sensors used in RPM systems which require high data transfer rates. Not only do these sensors need higher data transfer rates, but also multiple instances of such sensors may be deployed in one home, requiring much higher bit rate for continuous monitoring.

Table 3.1: Sensors and Data Transfer Rate Requirements.

\begin{tabular}{|l|l|}
\hline Sensor & Data Rate \\
\hline Microphone & $\sim 350 \mathrm{Kbps}$ \\
\hline Bed and Floor mat & $\sim 200 \mathrm{Kbps}$ \\
\hline Video & $\sim 1 \mathrm{Mbps}$ \\
\hline Infrared Camera & $\sim 1 \mathrm{Mbps}$ \\
\hline
\end{tabular}

The current RPM systems employ a distributed data processing architecture where most of the data processing and storage is done in the local node due to low data transfer rates available with existing $2 \mathrm{G} / 3 \mathrm{G}$ networks, as seen from Table 2.7. Doing signal processing at the local node can reduce the data rate requirement from Mbps range to $\sim 100$ bps. 


\subsection{Distributed Data Processing and Storage Architecture:}

In distributed architecture for the RPM system all the data processing and storage is done at the local node in the patient's home. The local node provides the backup storage for the sensor data. The trend analysis as well as emergency alert generation maybe located in central node or split between the central and local node. Central processing node stores the patient status results and provides access to the patient medical records and status information to clinicians via internet and GUI. Figure 3.1 shows the architectural diagram of distributed processing and storage RPM system.

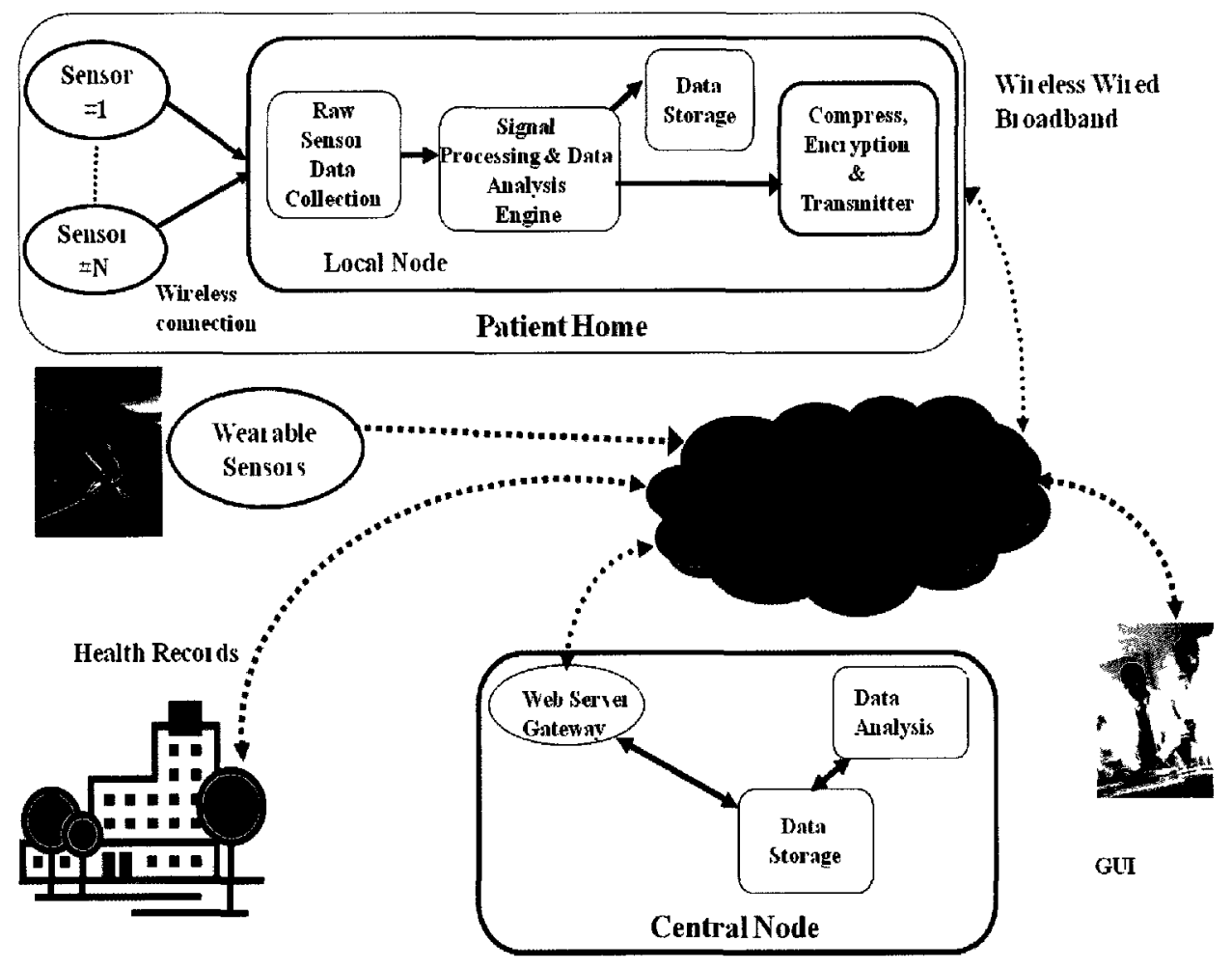

Figure 3.1: Distributed Data Processing and Storage Architecture

The distributed architecture allows non real-time critical data to be monitored in a store and forward mode. In store and forward mode the sensor data is stored on a local device and processed and/or transmitted from the local node on a periodic basis or at 
specified times. Store and forward method has advantage over real-time processing and/or transmission systems since there is no continuous data transmission and system is more robust in relation to any network instability, e.g. loss of network connectivity and network congestion.

In store and forward mode data can be transmitted at non-peak hours reducing network congestion issues at peak hours, allows for retransmission due to pocket loss and more complex encryption algorithms. This allows for better network bandwidth utilization.

The advantages of the RPM system using distributed processing architecture are

- Most of the processing is done at the local node and only clinically relevant information is transmitted to the central node. This significantly reduces the bandwidth requirement for the connecting network between the local and the central node. Since raw data rate is orders of magnitude greater than processed data. It reduces the data loss due to network congestion or temporary loss of connectivity.

- Sensors could have embedded processors to do the data processing. Embedded processor provides the signal processing before data transmission which could reduce the data rate of the transmitted signal. This will help increase the sensor battery life since transmitting data puts the maximum drain on battery.

- Non real-time critical data can be transmitted using store and forward mode allowing transmission at non-peak hours reducing network congestion issues. 
Some of the disadvantages of distributed architecture are:

- Every sensor (in home and outside) data processing hardware/software must be installed for each patient. This will increase the cost of the system.

- More hardware/software components lead to higher the risk of failures and increased need to access patient's home for maintenance.

- Any changes to hardware/software signal processing components requires access to the patient home for upgrade. This is costly especially when large number of RPM systems is deployed.

- Different RPM system vendors may use standardized sensors but may employ variety of signal processing techniques and corresponding wide variety of hardware and software. If the patient wants to change the RPM system vendor the old setup needs to be ripped out and new sensor and signal processing hardware/software installed. 


\section{Chapter 4: Proposed RPM System Architecture}

\subsection{Proposed Centralized Data Processing and Storage Architecture for the RPM System.}

Since RPM systems upload the patient data to a central node, the upload speed of the underlying network is critical for RPM systems. $4 \mathrm{G}$ wireless networks offer significantly higher data rates compared to current $2 \mathrm{G}$ and $3 \mathrm{G}$ technologies. With its high data transfer rate, $4 \mathrm{G}$ network opens the door to feature rich and comprehensive RPM systems with the ability to monitor various chronic and acute conditions continuously.

The current architectures for the RPM systems are limited by the capacity of the wireless network for transmitting monitored patient data.

This thesis proposes a centralized data processing and storage architecture for the RPM system that meets the objectives of the RPM system. The proposed architecture utilizes the high bandwidth available with wireless network technologies like current $4 \mathrm{G}$ and beyond. The proposed RPM system architecture meets all the design requirements. The proposed architecture is scalable allowing it to be used for monitoring with different sensor configuration.

Figure 4.1 shows the proposed RPM system architecture. 


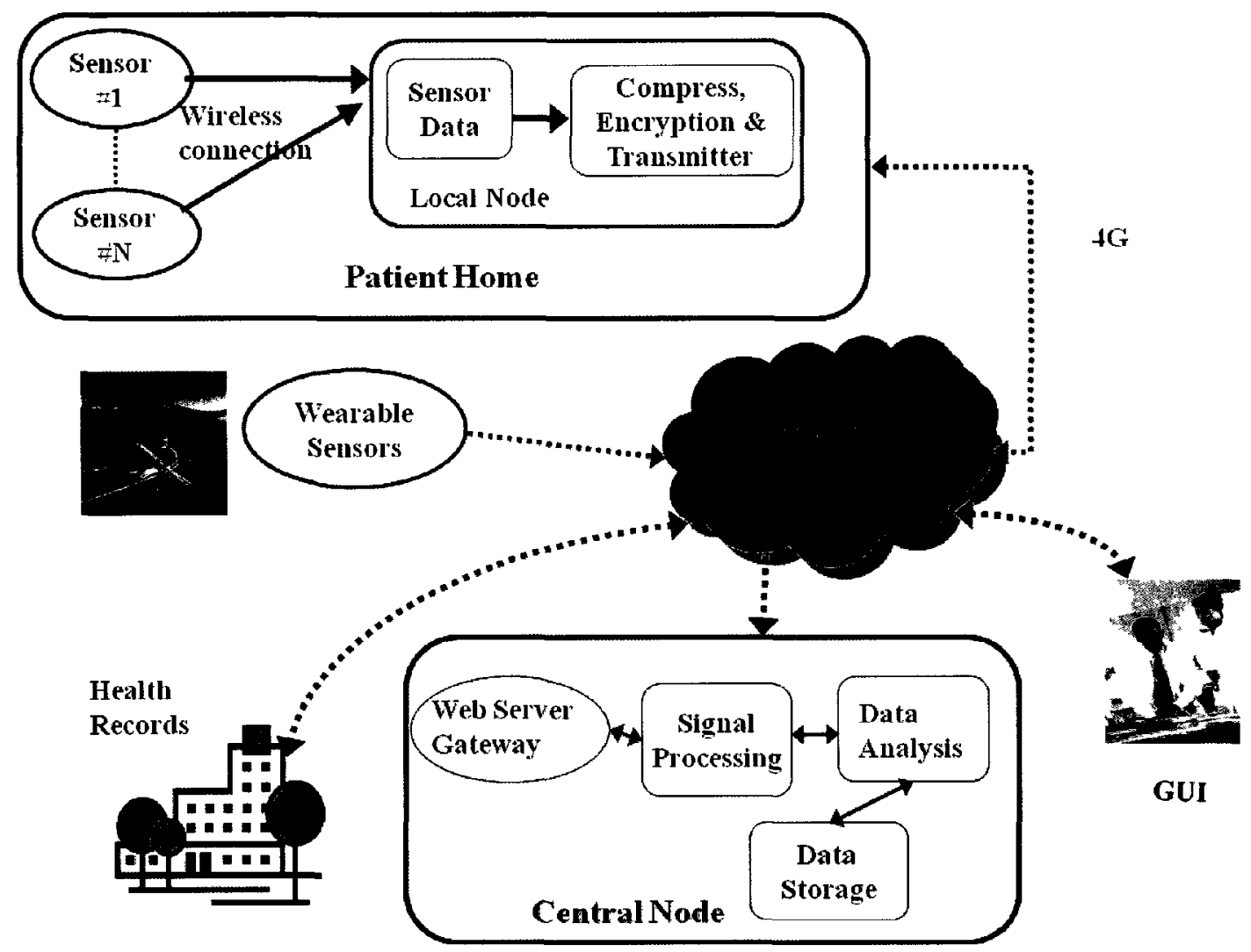

Figure 4.1: Centralized Data Processing and Storage Architecture

The main features of proposed architecture are:

- All of the signal processing and storage is done at the central node.

- The local node is only responsible for transmitting raw sensor data over the network after compression and encryption.

- Architecture requires a high capacity wireless network since the raw data rates, as seen from Table 2.1, can be very high.

System consists of sensors to collect biosignals as well as environmental data. The number of sensors used depends on individual patient needs. The sensors and the local node form a wireless personal area network (WPAN). The sensor data is sent to the local node using wireless protocols like Bluetooth LE or IEEE 820.15.4/Zigbee. With the 
proposed architecture it is possible to use cell phone or a PDA as a local node given low processing requirements in the local node. Use of cell phones like iPhone/Blackberry and PDAs is widespread in today's society. Use of cell phone/PDA significantly reduces the cost of the local node maintenance as well as provides ease of software or hardware upgrade. Cell phone/PDA based local node will transmit raw signal data directly to the central node using wireless network.

The application on the local node may assign different priorities to different sensor data packets based on its criticality. This will ensure that higher priority signals are sent earlier before lower priority signals. Protocol used for high priority signals may include acknowledgement and retransmission. The priorities can be set by healthcare providers based on individual patient condition.

The central node consists of a database to store data from all the local nodes, a signal processing platform to process and analyze all raw data. It may also be connected to the patient EHR so patient specific targets, treatment protocol can be used in data analysis. The processed data and EHR can be used along with a Clinical Decision Support System (CDSS) to provide healthcare providers with up-to-date patient status, trends in condition, diagnostic option and can highlight significant changes. The central node also has Web based GUI for allowing healthcare providers to access the patient data locally or remotely using Internet. All the data storage is done in the central node increasing the data security as well as reducing the cost of data backup.

Figure 4.2 shows a model of proposed RPM system architecture. 


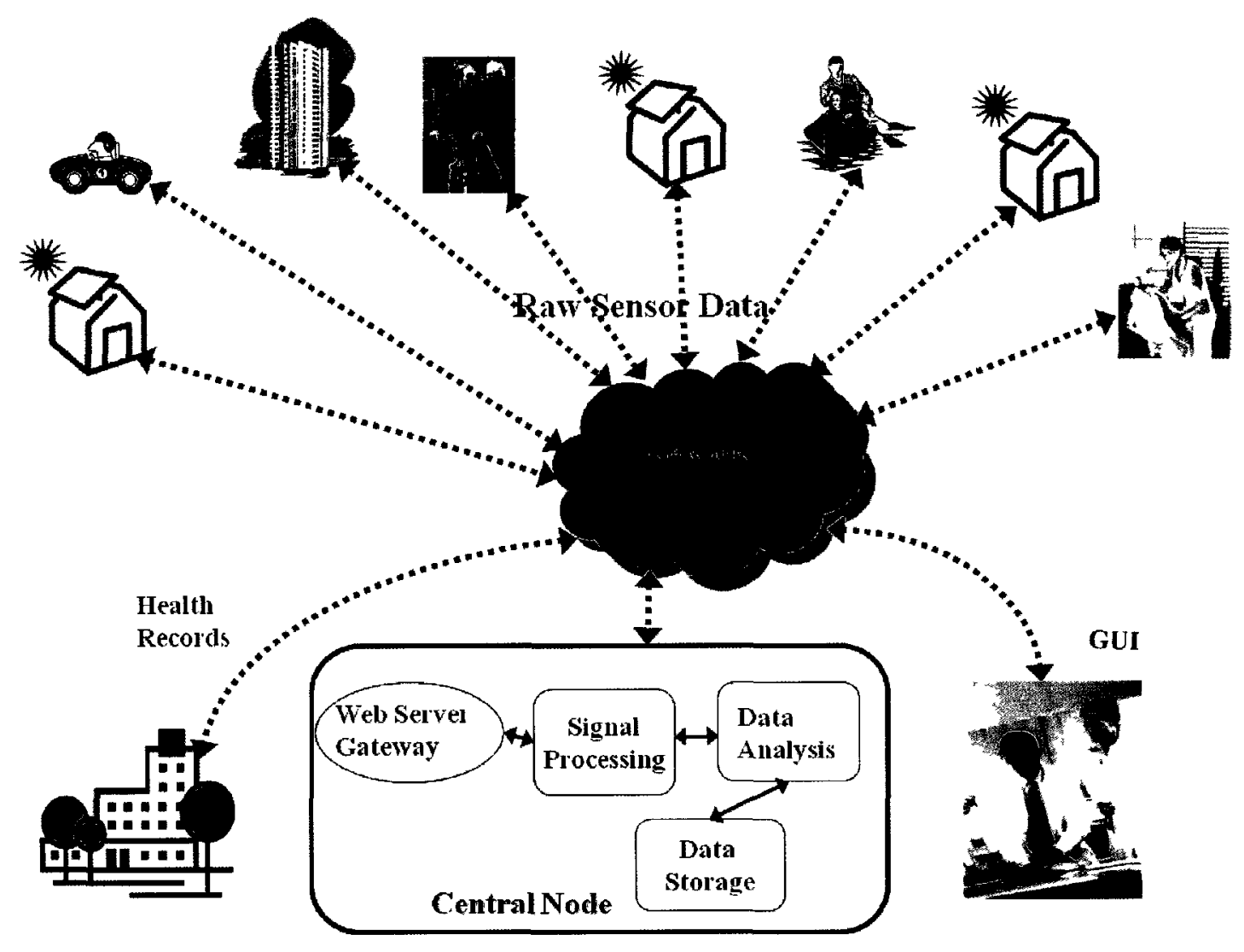

Figure 4.2: Model of Centralized Data Processing RPM System.

Keeping the number of system components in the local node to a minimum will increase reliability and reduce the overall maintenance cost since maintaining components in the central location is much cheaper.

The RPM system hardware and software will be upgraded over time to replace existing hardware or software with newer versions or adding new components. The upgrade process needs to be nonintrusive and should not affect system reliability. For the RPM system architecture that does most of the signal processing on the local node in the patient's home, upgrades cost will be higher and very intrusive.

The centralized architecture is easier and cheaper to maintain since it reduces the number of components at the remote patient site. 
This architecture requires high capacity wireless network since raw data from sensors is sent to the central node with no or minimal pre-processing.

\subsection{Comparison of Distributed and Centralized Architecture}

The Table 4.1 shows the various system requirements for the two architectural frameworks described above.

Table 4.1: Comparison of the RPM system using Distributed and Centralized Data Processing Architecture.

\begin{tabular}{|c|c|c|}
\hline $\begin{array}{c}\text { RPM System Architectural } \\
\text { Criteria }\end{array}$ & $\begin{array}{c}\text { Distributed Data } \\
\text { Processing and Data } \\
\text { Storage }\end{array}$ & $\begin{array}{c}\text { Central Data } \\
\text { Processing and Data } \\
\text { Storage }\end{array}$ \\
\hline $\begin{array}{c}\text { Cost: Installation, } \\
\text { Maintenance, Upgrade. }\end{array}$ & High & Low \\
\hline Scalability & Medium & High \\
\hline $\begin{array}{c}\text { Network BW and Data } \\
\text { Compression }\end{array}$ & Low & High \\
\hline $\begin{array}{c}\text { Network QoS and Reliability } \\
\text { High (Data could be } \\
\text { retransmitted) }\end{array}$ & $\begin{array}{c}\text { Low (Since raw data } \\
\text { is transmitted) }\end{array}$ \\
\hline Wireless Technologies & 2G/3G/4G & 4G and higher \\
\hline Data Security & High & High \\
\hline Real-time Signal Processing & High & High \\
\hline Sensor Battery Life & High & High \\
\hline
\end{tabular}

As seen from Table 4.1 major differences in Distributed and Centralized architecture requirement is 1) Network BW and QoS 2) Cost. The centralized architecture for the RPM system also has significant advantages over distributed architecture in terms of 1) Scalability and Flexibility 2) Mobility 3) Ability to migrate to newer computing models like Cloud Computing. Following sections provide a detail comparison of the two architectures for the differences mentioned here. 


\subsubsection{Network BW and QoS Requirements}

End to end data transfer rate between the local node at the patient's home and the central node is one of the factors in design of a wireless RPM system. Not only these sensors need higher data transfer rates, multiple of such sensors may be deployed in one home requiring much higher bit rate in the range of few Mbps for continuous monitoring.

Bandwidth available over network can be limiting factor for the RPM system. A network connection may traverses multiple intermediate networks engineered for different specification. End-to-end bandwidth of a network connection is limited by the bandwidth of any path of network connection and may not be guaranteed.

Low data transfer rates that existing $2 \mathrm{G} / 3 \mathrm{G}$ networks offers either limits the RPM systems to low bit rate sensors or needs most of the signal processing to be done at the patient's the local node.

In Distributed data processing architecture most of the signal processing is done at the local node and will reduce the data transfer rate requirement from Mbps range to $\sim 100$ bps for the different conditions being monitored at the patient's home.

Centralized architecture requires raw data to be sent to the central node for processing hence requires high capacity broadband network. Only WiMax/LTE/4G networks are capable of providing such high capacity. While such networks are being introduced they are not widely deployed.

Another major factor is the QoS requirement for the RPM system. Packet loss can have significant impact on quality of care provided by the RPM system and can result in loss of critical patient data. With low BW requirements it is possible to use more secure 
protocols like TCP with data retransmission without minimal delay even during peak usage.

While 4G network does offer high BW the congestion during peak periods is not clear due to its limited penetration. Newer QoS protocols are being introduced for IP network but end to end network meeting the requirements of those protocols is still in inception stage.

\subsubsection{Cost Requirements}

The cost of installation, maintenance and upgrade the RPM system can be significant, especially given the reliability requirements of an RPM system. The system architecture has a significant influence on the installation, maintenance and upgrade costs.

Distributed data processing architecture does most of signal processing at the local node and correspondingly has more components at remote patient site. Maintenance cost for distributed data processing architecture will be higher due to higher number of components with greater chance of failure. Maintaining system at remote site is more expensive and will be one of the major factors influencing overall maintenance cost.

The proposed centralized architecture shown in figure 4.2 where most the processing is done at the central node is easier and cheaper to maintain since it reduces the number of components at remote patient site.

The RPM system hardware and software will be upgraded over time to replace existing hardware or software with newer versions or adding new components. The upgrade process needs to be nonintrusive and should not affect system reliability. For the RPM system architecture that does most of the signal processing on the local node in the patient's home, upgrades cost will be higher and very intrusive. 
While sensor installation is one time process it is most likely that data processing algorithms for the sensor data will change more often either due to modification, bug fixing or new algorithms being introduced for the same sensor data. So ease of applying software upgrades with minimal interruption to the patient's life is critical.

The upgrade process for proposed architecture is significantly simple, less intrusive and cost effective since most of the upgrades will not require access to the patient home.

Centralized signal processing architecture reduces the cost of installation since components to be installed at each patient are reduced and maintaining components in central location is much cheaper.

While there is no direct data to evaluate cost benefits of distributed vs. centralized architecture for RPM systems the research in to the client/server and thin computing model has shown that the hardware and software cost (purchase, install, maintain) can be reduced by $40 \%$ as well as achieving $30 \%$ reduction in operation costs [122], [123]. The centralized architecture for the RPM system will provide similar savings compared to distributed architecture reducing the price of the RPM system and helping it wider usage.

\subsubsection{Scalability and Flexibility}

The proposed architecture is easily scalable since adding new sensor does not require any modification at the local node. Since no signal processing is done at local node it is very easy to isolate failure from the central node. A failed sensor can be easily turned off or put in sleep mode from central node. This allows integrity of the system to be maintained with least intervention. System is highly flexible for use under different scenarios like patient home, long term care facility; retirement home etc since components at the patient site is minimized. 
Keeping the number of system components in the local node to a minimum will increase reliability and reduce intrusion into the patients' lives.

\subsubsection{Mobility}

Impact of the RPM system on the patient's mobility needs to be minimized. Mobility discussed here relates to ability to leave residence for overnight stays, measured in hours and days rather than months. The RPM system that does not affect the patient mobility will ensure that the patients are not restricted to their residence. RPM system setup should be simple enough so that they are able to leave their residence for visit to their family/friends as a part of their daily living pattern. Patient's mobility will directly affect the RPM system acceptance. Any RPM system that limits patient mobility will be negatively viewd by patients.

The RPM system setup needs to be simple so that critical sensors can be moved to new location with no or minimal help and interruption if needed. The proposed centralized architecture allows minimal components needed at the patient's home and simplifies the required setup. This will significantly enhance patient mobility compared with distributed processing architecture allowing better patient mobility.

\subsubsection{Cloud Computing and Proposed RPM System Architecture}

Cloud computing is seen by many as the future direction on how computing services will be provided. Though cloud computing means different things to different users, in essence it is a way of delivering computing services over internet [124]. It can be characterized as being scalable, on-demand, internet based and pay-as-you go pricing. Users only pay for the services they use. Cloud computing service provider is responsible 
for software and hardware platform. Cloud computing service providers offer Softwareas-a-Service (SaaS), e.g. Salesforce (CRM), Netsuite (N), and Google (GOOG) [124].

One of the advantage of cloud computing is users do not have to buy technology (hardware and software) and constantly upgrade it as it becomes obsolete in few years, significantly reducing upgrade and maintenance costs.

Mobile devices especially smartphones are becoming extremely popular. Collaboration software on mobile devices allows users to access and perform content editing, management where content is stored centrally in a cloud providing true mobility. Ability to access SaaS applications from user mobile devices using a mobile device-based browser to access the cloud will become a major driving force in near future [125]. This is especially true with the advent of high bandwidth networks like $4 \mathrm{G}$ and up along with the 4G-capable mobile devices. Figure 4.3 shows an architectural view of cloud computing model.

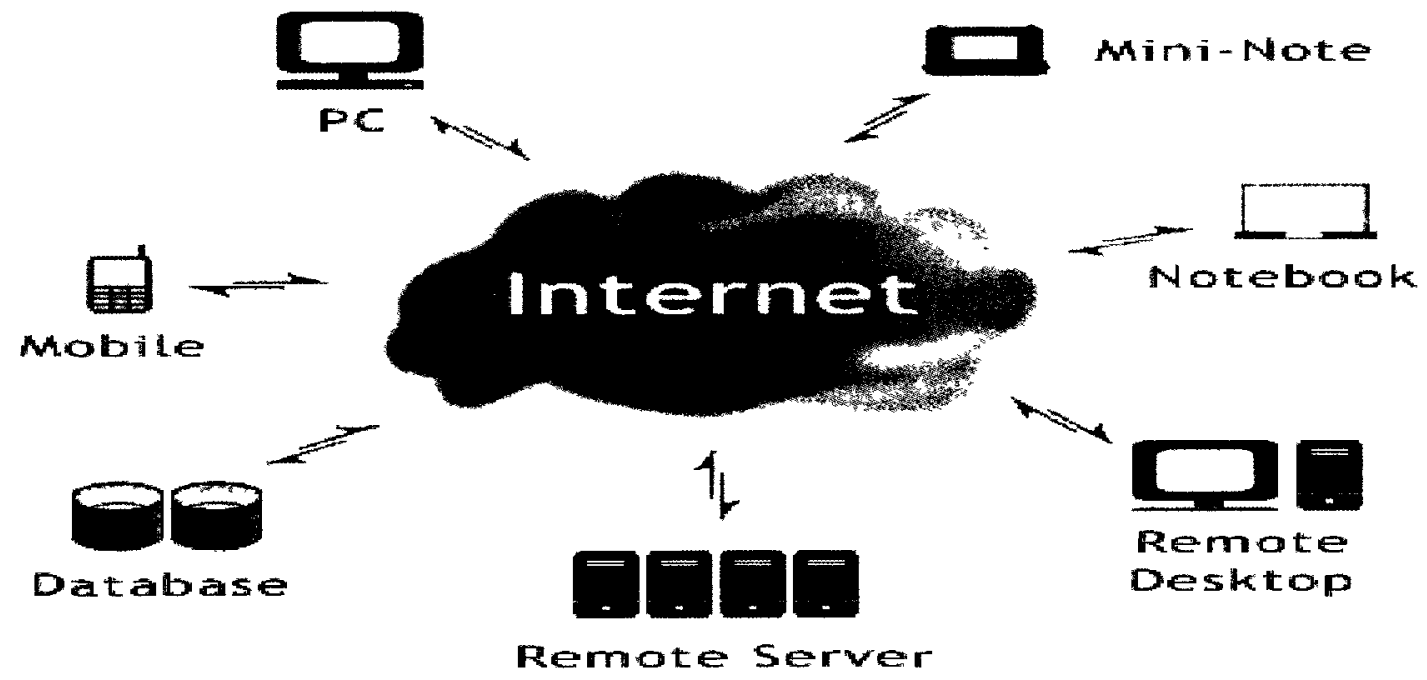

Figure 4.3: Cloud Computing Model, reproduced from: [126]. 
The proposed centralized data processing and storage architecture lends itself for very easy adoption to cloud computing model. The central node hardware platform, signal processing and storage functionality can be considered as a service delivered from the cloud such as Platforms-as-a-Service (PaaS) for hardware and Software-as-a-Service (SaaS) for signal processing and data storage. This allows the central node implementation to migrate from a traditional on-premise infrastructure model to a cloud model as shown in Figure 4.4. Local node is a simple mobile device like PDA or cellphone connected using $4 \mathrm{G}$ to central node.

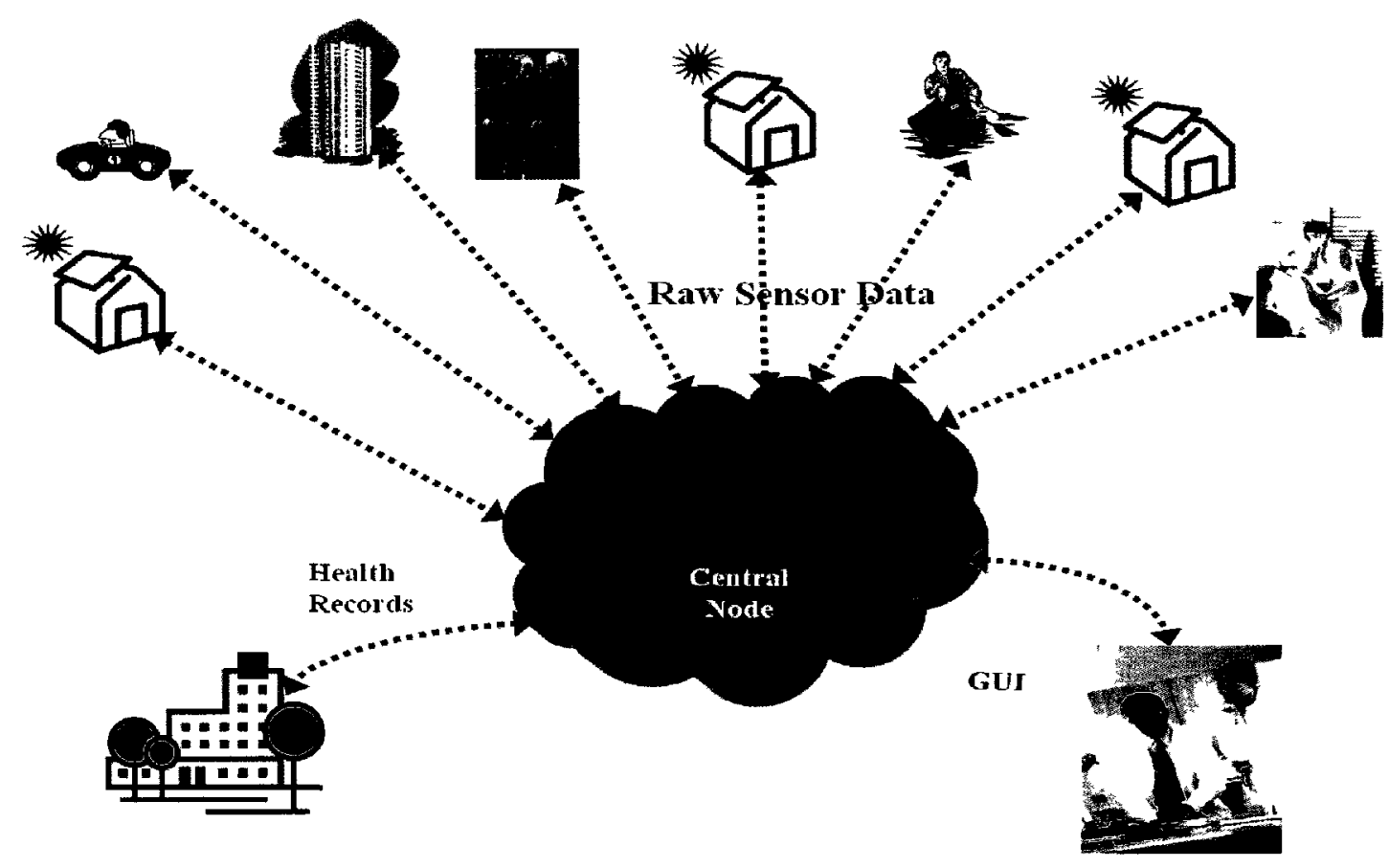

Figure 4.4: Centralized Data Processing RPM System using Cloud Computing.

Cloud service providers host all of the hardware and software needed to provide services, eliminating the need for healthcare providers to maintain data center space and facilities. Some of the advantages of using cloud computing for centralized data processing RPM system are: 
- No data center space and maintenance required

- Reduce obsolescence since service provider is responsible to maintain the platform

- Expenditure for capital, power consumption, hardware devices, space, onsite IT department for maintenance and upgrades is minimized.

- Platform independence allows users to access systems regardless of their location and what device they might be using for access.

- System administration is streamlined.

- Upgrades and backup service is provided by service provider's global accessibility.

The service provider needs to ensure that platform is fault tolerant so that hardware or software failures do not affect patient's well being. Similarly it must ensure the security and privacy of the patient data.

\subsubsection{Summary}

The proposed centralized RPM system architecture is capable of integrating multiple sensor data, is scalable and requires very little user intervention. This is achieved by centralizing the entire signal processing of the raw sensor data in the central node. It utilizes high bandwidth that will be available with $4 \mathrm{G}$ and beyond wireless network. The proposed architecture can be very easily incorporated with the emerging computing technologies like cloud computing.

As RPM systems are widely deployed the cost of maintaining them will play a critical role in the choice of the RPM system architecture. In future more and more sensors requiring complex signal processing will be employed for the RPM. If signal processing 
is done in the local node at the patient site an upgrade or patch fixes for the software will have to be done in each patient home either physically going there or via remote login both will increase system down time and will significantly add to the maintenance cost. The proposed centralized architecture provides ease of maintenance, reduced cost of upgrade and maintenance. Since raw data is available in the central location newer processing algorithms can be easily tested with stored raw data before being released in the field. Healthcare providers can draw upon the raw data in case they require it for more details.

Based on installation, maintenance and upgrade cost as well as reduced computing and storage costs via use of cloud computing the proposed centralized signal processing the RPM system architecture performance significantly better than other architecture utilizing the local node signal processing. It also allows much higher mobility for the patients to travel to another city.

There are multiple commercial vendors providing RPM systems. With RPM systems set to become integral part of healthcare plan in future the number of vendors providing RPM systems will grow significantly. Centralized architecture provides significant ease of changing vendors since only sensors are installed at the patient's home with all signal processing off loaded to the central node. Centralized architecture using cloud computing will significantly improve the ability to switch between vendors.

While network transmission costs will be higher for centralized architecture, it is expected that the cost per bit over $4 \mathrm{G}$ network will be significantly lower than $3 \mathrm{G}$ network. 


\section{Chapter 5: Proposed Adaptive Information Content Management and Buffer Caching Design}

Network congestion and loss of network connectivity is a serious problem in wireless network [38], [89], [84], [90]. High speed internet has made video/audio streaming and online gaming applications easily accessible, substantially increasing the demand for bandwidth. The pattern of usage for streaming applications is very different than the traditional peak hours of the past. With newer applications high network usage is around the clock [109], [110]. Today's wireless networks are getting highly congested. Operating companies are in a race to upgrade their existing networks to $4 \mathrm{G}$ to meet the exploding bandwidth demand [107].

Real-time patient monitoring requires that network connectivity between sensors, the local nodes and the central node is constantly available. Loss of connectivity and high network latency due to network congestion will have a significant impact on the QoS offered by the RPM system [84], [89], [90]. It will also result in loss of real-time data, and can affect patient health when the condition being monitored is acute.

While no real-time continuous RPM system can survive catastrophic network failures, the RPM system design must be robust enough to survive a certain level of network congestion and temporary loss of connectivity without losing critical patient data. 
While high data rates available with $4 \mathrm{G}$ wireless network makes implementation of the proposed centralized signal processing architecture for the RPM system feasible such a RPM system must be robust enough to be able to survive adverse network conditions due to network congestion or connection loss.

\subsection{Background}

Buffering can be used to hold data when network congestion or loss occurs till network connection or capacity is restored. But buffer lengths have limits and buffers will overflow once they are full, resulting in data loss, e.g. at $1 \mathrm{MBbps}$ data rate RPM system with a 10 MByte buffer will overflow in $10 \mathrm{sec}$. Simple buffering techniques result in the irrecoverable loss of all information content from the sensor signals when the buffer overflows.

While it is possible to eliminate congestion by over provisioning a network it is practically impossible to eliminate end-to-end congestion due to multiple networks that may be involved in a connection. Most of the networks are not over provisioned due to cost involved [111].

Another solution for achieving network reliability is having redundancy in network connection. This can be achieved by having network connectivity via two different network providers. One would act as a primary connection and second one as a backup, used in case first network is not available or congested. This will increase overhead and cost of the system and is only viable where complementary wireless networks (4G) are available.

Newer QoS protocols like Classifier, Marker (DiffServ, IPv6), and Scheduler etc as mentioned in Chapter 2 are being implemented in IP network for real-time applications. 
Yet end to end network meeting the requirements of those protocols is still in inception stage.

\subsection{Proposed Adaptive Information Content Management (AICM) Method}

Loss of critical patient information when available network capacity is below the requirement or due to temporary loss of network connectivity can be prevented by intelligently reducing the transmission data rate.

This thesis proposes a new "Adaptive Information Content Management (AICM)" method along with sender buffer caching scheme for centralized data processing RPM system that is robust and highly survivable under adverse network conditions. The proposed AICM method reduces the data rate of the RPM system by reducing the information content being transmitted to the central node. The proposed design is scalable and adaptable for different network and sensor configurations and can extend the survivability time of the RPM system without losing critical patient data.

The proposed AICM method along with sender buffer caching, is very generic and not restricted for use with the proposed centralized RPM system architecture only. It can be used for any RPM system architecture to make it robust against network congestion and/or failure and resulting loss of critical patient data.

\subsubsection{RPM System Architecture using the AICM and Buffer Caching}

The AICM method collects the information available from all of the sensors and partitions it into multiple levels. Pre-processing progressively reduces the information content from one level to the next, such that lower information content leads to a lower data rate, i.e. 
Information content at Level L-1 > Information content than Level L

and

Data Rate at Level L-1 > Data Rate at Level L

The data rate reduction factor at each level is defined as $D_{1}$ and $D_{1+1}>D_{1}$. Level 1 is considered to hold the full raw data so that

$$
\mathrm{D}_{1}=1
$$

Each level has a separate send data buffer and data at each level is cached in buffer space allocated for that level. The output from the RPM system can be adaptively selected from buffers at different levels. By selecting different send buffers the RPM system can adaptively select the information content that is transmitted to the central node. The selection of data buffer for sending the data is done such that the transmission data rate at the selected level is less than the available network capacity. This adaptive selection of data buffer and corresponding transmitted data will ensure that the critical patient data is not lost due to network congestion and/or temporary loss of network connectivity.

The information content at each level needs to be carefully selected to ensure that critical patient data is still retained during network congestion or connection loss. The choice of which data is critical for a patient and needs to survive adverse network condition will be dependent on the patient's medical conditions being monitored, sensors used and the network capacity and is a RPM system design criteria.

Figure 5.1 shows the architecture of the RPM system using adaptive information content management. 


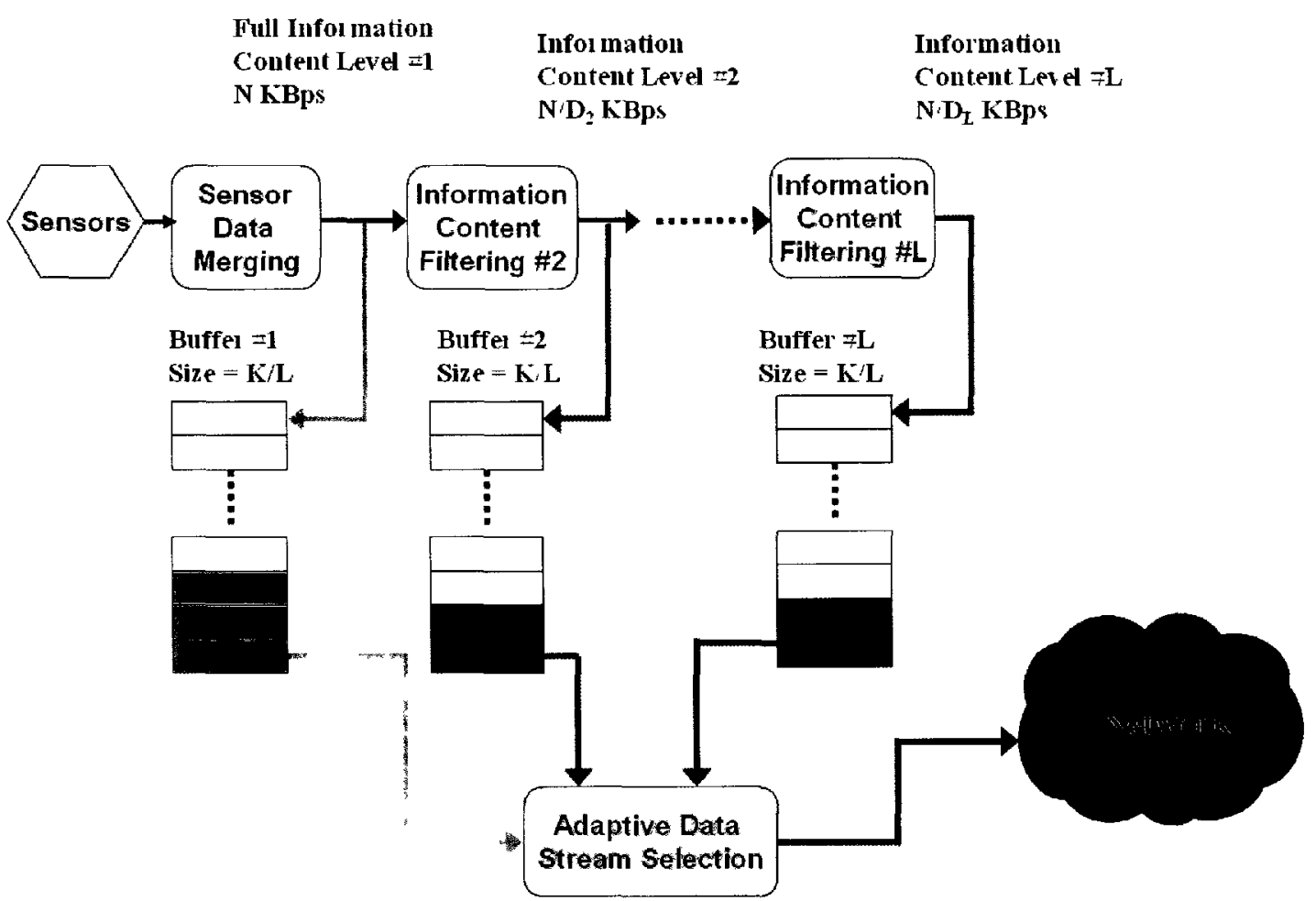

Figure 5.1: AICM RPM system using static multi buffering scheme.

The allocation scheme used to allocate buffers for each level is a static buffering scheme where total available buffer of size $\mathrm{K}$ Bytes is divided equally between all the LLevels. So buffer size at each level is given by $\mathrm{K} / \mathrm{L}$.

The total "Survival Interval" (SI) of the RPM system using the AICM method is defined as the length of time in seconds that the system will be able to retain patient information during a full connection loss. So if system suffers connection loss greater that SI then loss of critical patient information will occur. The SI is given by

$$
\mathrm{SI}=\left(\mathrm{K} /\left(\mathrm{L}^{*} \mathrm{~N}\right)\right)^{*} \sum_{i=1}^{L} D_{i}
$$

Where $\mathrm{K}$ is the total buffer size available, $\mathrm{L}$ is the number of levels, $\mathrm{D}_{1}$ is the data rate reduction at Level $\mathrm{i}$, and $\mathrm{N}$ is the original full data rate in $\mathrm{KBps}$. 


\subsubsection{Example of the AICM Method for $L=3$}

In this section the concept of AICM is illustrated using a RPM system with three levels for the AICM method, $\mathrm{L}=3$. Though $\mathrm{L}=3$ is used as an example the design methodology is extendible to any number of levels $L(L>1)$.

Figure 5.2 shows an adaptive RPM system with 3 information content levels $(L=3)$ and corresponding 3 static buffers with a total buffer size of $\mathrm{K}$ Bytes. The data rate reduction factor at each level is defined by variable $D_{1}$ where $i$ is the level number, e.g. $D_{1}=1$ for level \#1, and $D_{3}>D_{2}>D_{1}$. Data from each processing step is being stored in 3 separate $\mathrm{K} / 3$ Byte buffers. The data rate for full information content is defined as $\mathrm{N}$ KBps.

No Congestion NetworkData Rate > N KBps

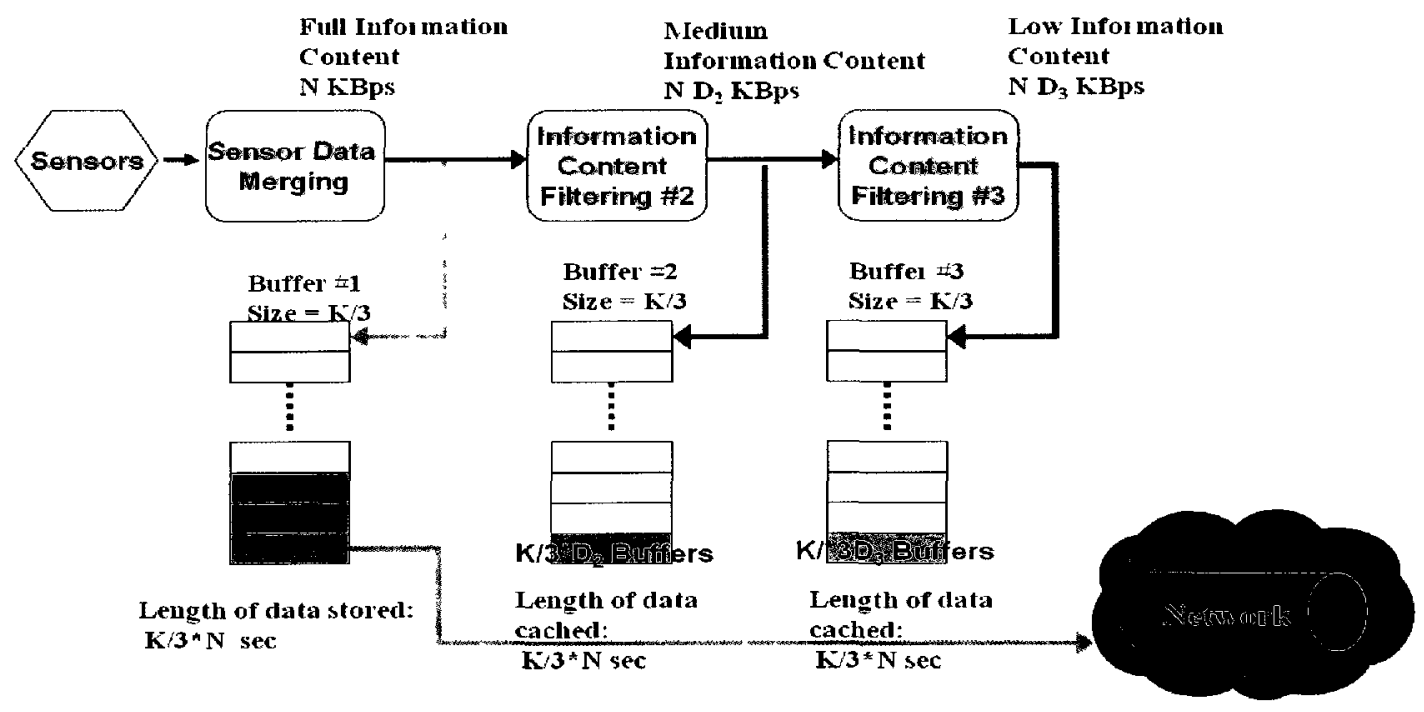

Figure 5.2: Adaptive information content and static multi buffer RPM system with little or no network congestion.

When network capacity is greater than N KBps the Buffer\#1 is selected for data transmission and there is no buffer overflow from Buffer \#1 and full information content 
is sent to the central node. While data is being transmitted from Buffer \#1, lower information content data is being cached in buffer $\# 2$ and $\# 3$ on an ongoing basis. The length of the data being cached in Buffer\# 2 and Buffer\#3 is equal to:

$\mathrm{K} / 3^{*} \mathrm{~N}$ seconds

This ensures that buffers can be switched dynamically between different Levels without loss of continuity.

If the network capacity drops below N KBps, Buffer \#1 will overflow. When Buffer \#1 overflow occurs, the system adaptively starts transmitting the data from Buffer\#2, where data rate is $N / \mathrm{D}_{2} \mathrm{KBps}$. This allows the RPM system to dynamically adapt to the lower available capacity of the network.

The information content in Buffer $\# 2$ is less than the original full raw data contents in Buffer\#1, but the system will survive for a longer time (by a factor of $\mathrm{D}_{2}$ ) than if the data rate was not reduced. Figure 5.3 illustrates the scenario. While data is being transmitted from Buffer\#2 data from Level 3 is cached in Buffer\#3. 


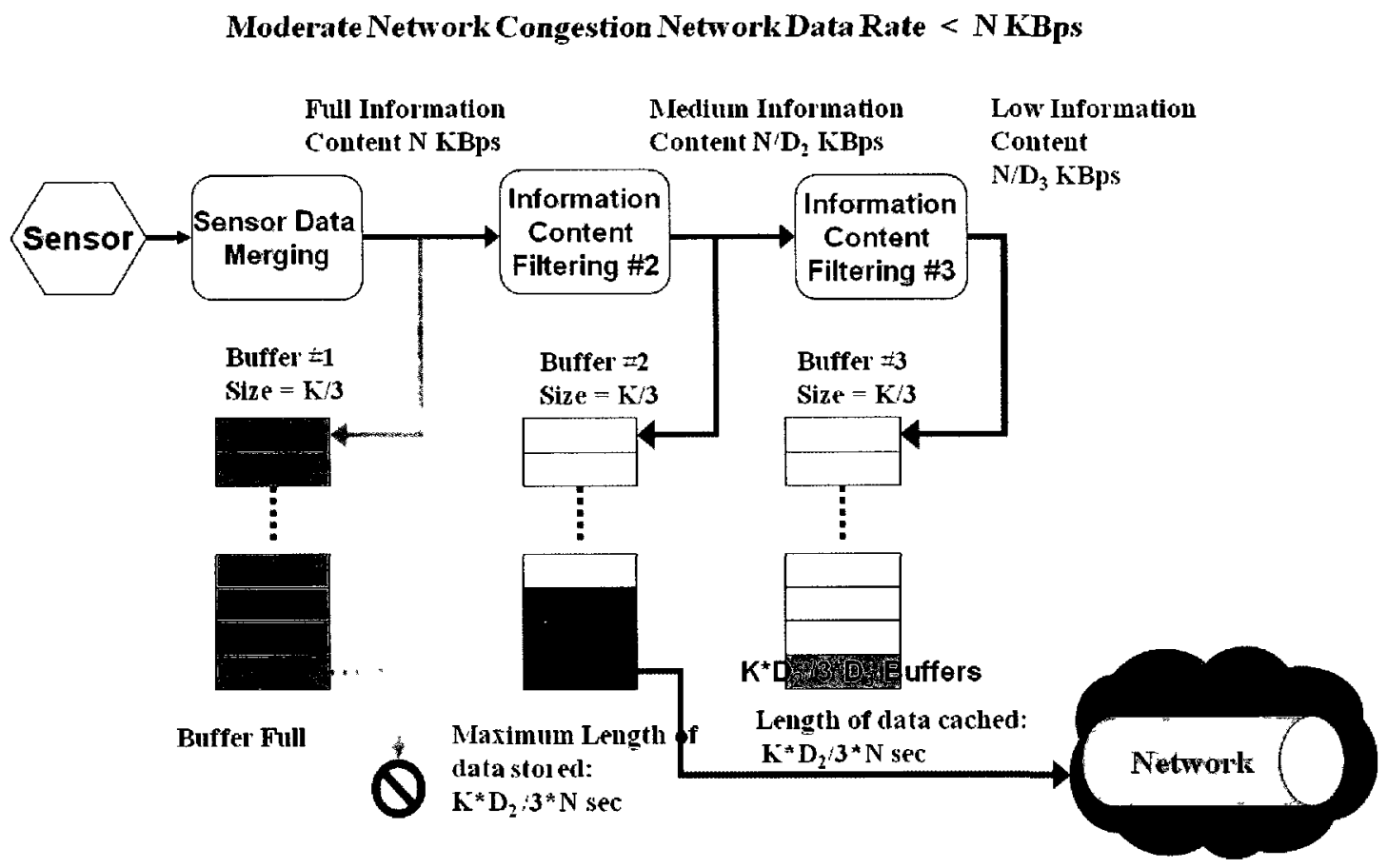

Figure 5.3: Adaptive information content and static multi buffer RPM system with moderate network congestion.

If the network capacity drops below N/D 2 KBps, Buffer \#2 will overflow. When Buffer \#2 overflow occurs, the system adaptively starts transmitting the data from Buffer\#3, which is at bit rate $\mathrm{N} / \mathrm{D}_{3} \mathrm{KBps}$, adapting to the lower capacity of the network. The data rate for Level 3 is lower by a factor of $D_{3}$ and the system will survive for much longer than it would for Level \#1 and Level \#2 information content. Figure 5.4 illustrates the scenario.

Ultimately a limit will be reached when network capacity drops below Level\#3 transmission rate, $\mathrm{N} / \mathrm{D}_{3}$, or when network connectivity is lost the Buffer\#3 will overflow resulting in loss of the patient data for all levels.

Depending on the choice $\mathrm{D}_{2}$ and $\mathrm{D}_{3}$ survivability interval (SI) given in equation (5.1) can be significantly increased for the RPM system using the proposed AICM method. 


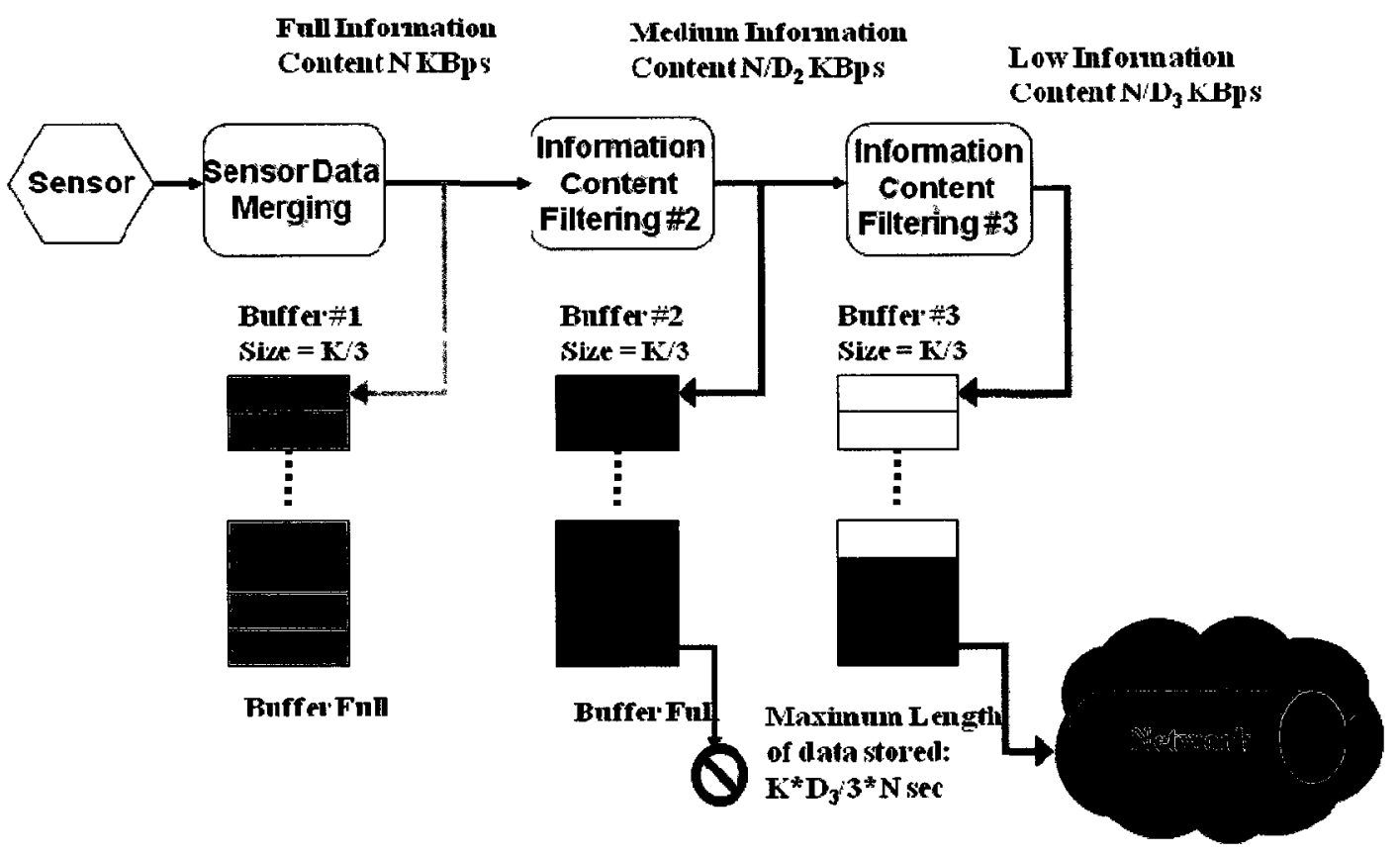

Figure 5.4: Adaptive information content and static multi buffer RPM system with heavy network congestion.

\subsubsection{Survivability Interval (SI) Evaluation for Different $D_{2}$ and $D_{3}$}

Equation (5.1) can be used to predict the SI of a RPM system for different values of $D_{2}$ and $D_{3}$ Two sets of values for $D_{2}$ and $D_{3}$ were chosen to illustrate how SI varies with $\mathrm{D}_{2}$ and $\mathrm{D}_{3}$.

For set 1

$$
\mathrm{D}_{2}=2: 100 \text { and } \mathrm{D}_{3}=2 * \mathrm{D}_{2}
$$

And for set 2

$$
\mathrm{D}_{2}=2: 100 \text { and } \mathrm{D}_{3}=10 * \mathrm{D}_{2}
$$

For $\mathrm{D}_{2}$ a range of values from low (2) to high (100) was chosen. The choice of $\mathrm{D}_{3}$ was made to illustrate how SI varies when the ratio of $D_{3}$ to $D_{2}$ is low (set 1 , ratio is 2 ) and 
when ratio of $D_{3}$ to $D_{2}$ is high (set 2, ratio is 10 ). The range of values chosen for $D_{2}$ and $D_{3}$ give fair demonstration of SI variability but equally extendible to other ranges.

For this example following are the assumption, though it can be applied to different values of $\mathrm{K} / \mathrm{N}$.

$$
\mathrm{K} / \mathrm{N}=1 \text { second }
$$

for both the AICM and non-AICM methods.

Using equation (5.1) Plots of SI versus different values of $D_{2}$ for set 1 and 2 were obtained. SI for non-AICM RPM system $=1$ second in both cases. Figure 5.5 shows the SI for $\mathrm{D}_{2}$ varying from 2:100 and $\mathrm{D}_{3}=2 * \mathrm{D}_{2}$ (set 1 ).

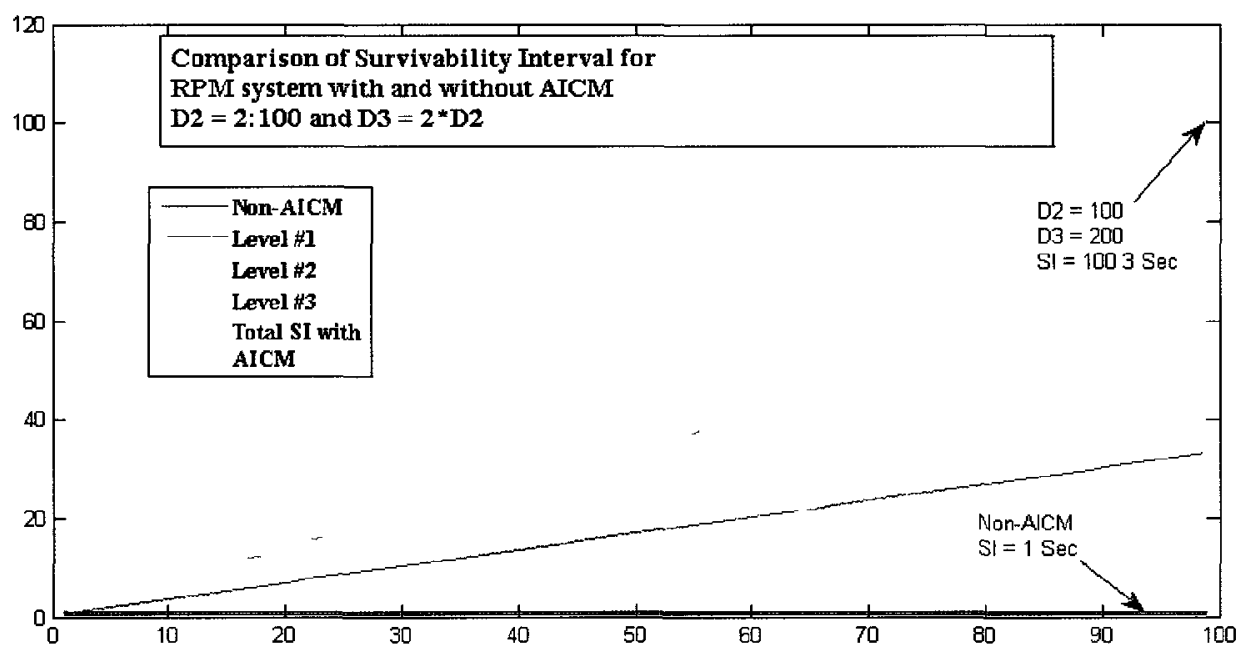

Figure 5.5: Comparison of Survivability Interval for the RPM system with and without AICM $D_{2}=2: 100$ and $D_{3}=2 * D_{2}$

As seen from Figure 5.5 for data point when $D_{2}=100$ and correspondingly $D_{3}=200$ SI is equal to100.33 seconds compared with 1 second for non-AICM method. Plot also indicates that as $\mathrm{D} 2$ increases (and correspondingly $\mathrm{D}_{3}$ ) SI of the AICM grows linearly. 
Figure 5.6 shows similar plot of SI for $D_{2}$ varying from 2:100 and $D_{3}=10^{*} D_{2}$ (set 2). As seen from Figure 5.6 for data point when $\mathrm{D}_{2}=100$ and correspondingly $\mathrm{D}_{3}=200 \mathrm{SI}$ is equal to 367 seconds compared with 1 second for non-AICM method.

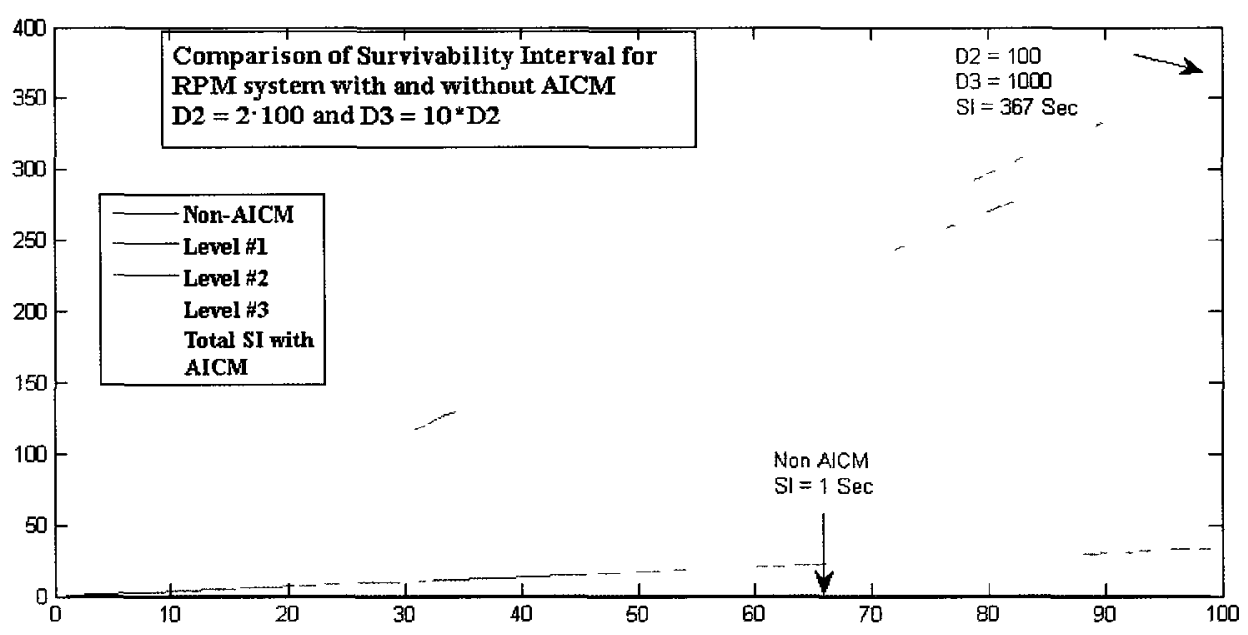

Figure 5.6: Comparison of Survivability Interval for the RPM system with and without AICM D2 $=2: 100$ and D3 $=10 * D 2$

It is also clear from Figure 5.5 and 5.6 that most gain in survivability for 3-Level system is obtained when D3 is much higher than D2.

\subsection{Summary}

A fully wireless RPM system maximizes patient mobility inside and outside home and provides portability. The reliability of wireless network becomes critical component for any RPM system using wireless technology. Despite the high bandwidth available with upcoming $4 \mathrm{G}+$ wireless networks, periodic network congestion as well as temporary loss of connectivity will continue to occur. Though varies network QoS like Guaranteed Service are likely to become available it may not be available over end to end connection. 
Temporary loss of connectivity and/or congestion will lead to data loss for the RPM system. The proposed AICM methodology for the RPM system can significantly improve the survivability performance of the RPM system under adverse network condition. The AICM method achieves better survivability by reducing the information content it is transmitting when network bandwidth is reduced or connection is lost. The reduction in information content leads to lower data rate for the sensor data being transmitted and hence improving the survivability interval of the RPM system.

Increased survivability time can be achieved using multiple levels of progressively reduced information stream from the local node. The number of information content levels used will depend on the sensors used, network capacity and the patient condition.

The AICM method ensures that while some data will be lost under overloaded network scenario, there is no complete loss of patient data. This provides for ability to retain critical patient data by discarding non-critical patient data. Thus it provides ability to trade-off transmission of patient data to meet reduction in network bandwidth due to congestion or loss of connectivity while still harvesting vital patient data. Use of the AICM method will become crucial as number of sensors employed in the RPM system increases with corresponding need for higher network bandwidth.

It is important to keep in mind that centralized processing architecture does very little processing in the local node which allows PDAs, cell phones to be used as the local node. Since information content reduction happens in the local node it is critical that any information content reduction method should not require intensive signal processing which cannot be handled by local nodes using PDA/cell phones. 


\section{Chapter 6: Proposed Contiguous Buffering Scheme for the AICM to Maximize Survivability Interval}

The AICM method described in Chapter 5 uses static buffering scheme where a separate buffer of fixed length is used for caching the sensor data at each information content level. While data is being sent from buffer at one level the data from other levels is being cached in their own buffers. This causes a large portion of buffer space to be left unused. It is possible to utilize the buffer space more efficiently. The driver for using buffer space more efficiently is to maximize the survivability of the RPM system.

In this chapter a different buffering scheme from the static buffering scheme used in Chapter 5 is proposed that will utilize buffer space more efficiently and maximize survivability interval and minimize information loss while using same buffer space as used in static buffering method (K Bytes) used in Chapter 5.

Proposed buffering scheme is a contiguous buffering scheme where buffers are used as needed rather than being pre-allocated. This buffer caching scheme uses the entire buffer space as one contiguous block. Figure 6.1 shows the dataflow for this contiguous buffering scheme. 


\section{Proposed Buffering Scheme to Maximize SI}

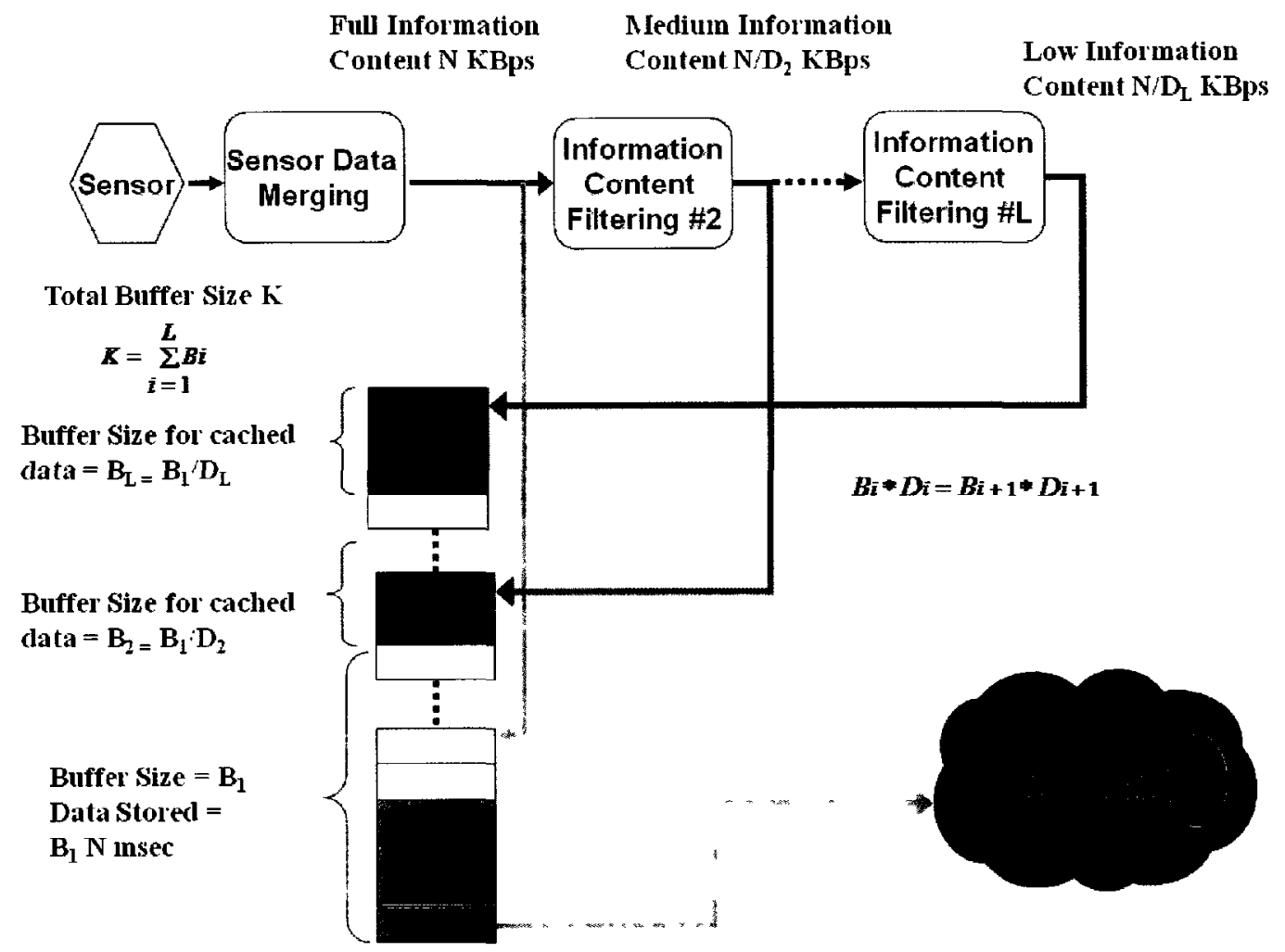

Figure 6.1: Data flow for AICM RPM system using contiguous buffering scheme.

Total buffer size $\mathrm{K}$ for this scheme is same as the static buffering scheme used in previous chapter. To prevent timing discontinuity in the information being transmitted the data from each level is stored in the section of the same buffer.

To understand how contiguous buffering scheme operates lets considers a scenario where data is being transmitted from level ' $i$ '. In this case the buffer is shared between levels ' $\mathrm{i}$ ' to ' $\mathrm{L}$ '. No data is stored for levels ' 1 ' to ' $\mathrm{i}-1$ '.

The first portion of the buffer for level ' $i$ ' named $B_{i}$ stores the data that is being transmitted over the network. The remaining buffer is partitioned proportionately so that there is no timing discontinuity in the data stored at each level.

The length of the data stored at each level is related by following equation, i.e. 


$$
B i * D_{i}=B i+1 * D i+1
$$

where $B_{1}$ is the buffer size for storing data or caching data at level ' $\mathrm{i}$ ' and data stored at buffer $B_{1}$ is given by

$$
B i=\frac{K}{\left(1+\sum_{m=i+1}^{L} D_{i} / D_{m}\right)}
$$

and

$$
K=\sum_{m=i}^{L} B_{m}
$$

Initially when sufficient network bandwidth is available contiguous buffering scheme transmits the data from Level ' 1 ' is stored in buffer $B_{1}$. When network bandwidth drops and buffer $B_{1}$ overflows entire buffer is reconfigured to store and cache data from level ' 2 ' and up only. The data is transmitted from buffer $\mathrm{B}_{2}$. So the buffer is disturbed among L-1 levels only. When buffer $\mathrm{B}_{2}$ overflows the buffer is again reconfigured to store and cache data from level ' 3 ' and up only. The data is transmitted from buffer $B_{3}$. So the buffer is disturbed among L-2 levels only. The process continues as each buffer overflows due to reduction in network bandwidth. 
Survivability interval for such contiguous buffering scheme with L-Level information caching is given by

$$
S I=\sum_{i=1}^{L} B_{i} / R_{i}
$$

Where,

$$
\begin{aligned}
& B_{1}=\text { Buffer length for level } i \\
& R_{1}=\text { Data rate at each level }\left(N / D_{1}\right)
\end{aligned}
$$

\subsection{Example of Proposed Contiguous Buffering scheme Functionality for $L=3$}

The functionality of the proposed contiguous buffer management scheme is explained in this section using 3-Level information content system $(\mathrm{L}=3)$ though it is extendable for any $\mathrm{L}>3$.

Figure 6.2 shows the block diagram of the contiguous buffer management scheme using $L=3$. When sufficient network bandwidth is available the raw data from level ' 1 ' is stored in buffer $\mathrm{B}_{1}$ and transmitted to the central node while data with lower information content is cached in a buffers $\mathrm{B}_{2}$ and $\mathrm{B}_{3}$ as seen in Figure 6.2. 


\section{No Congestion NetworkData Rate $>$ N KBps}

Full Information

Content N KBps
Medium Infol mation

Content N/D2 KBps
Low Infon mation Content $N / D_{3}$ KBps

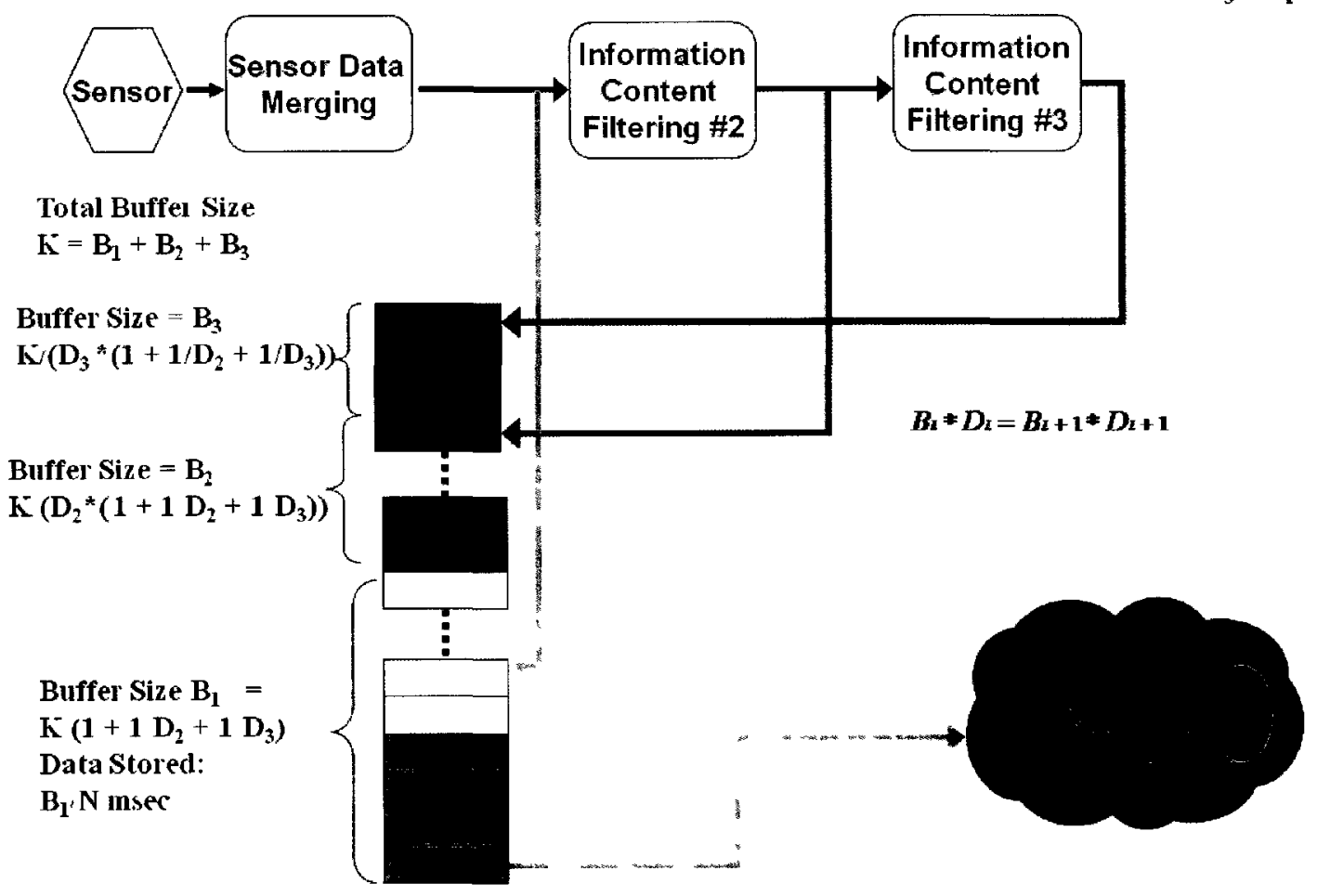

Figure 6.2: Adaptive information content and contiguous buffering RPM system with no or little network congestion.

In this case

$$
\begin{aligned}
& \left.\mathrm{B} 1=\frac{\mathrm{K}}{(1+1 / \mathrm{D} 2+1 / \mathrm{D} 3}\right) \\
& \left.B 2=\frac{K / D 2}{(1+1 / D 2+1 / D 3}\right) \\
& \left.B 3=\frac{K / D 3}{(1+1 / D 2+1 / D 3}\right)
\end{aligned}
$$


When network capacity drops below $\mathrm{N}$ KBps, the buffer $\mathrm{B}_{1}$ will over flow. In this case the data transmission will continue from Level \#2 with lower information content. Also all the buffering space will be reconfigured to reuse the buffer space previously used for storing data from Level \#1 as shown in Figure 6.3.

\section{Moderate Network Congestion Network Data Rate $<$ N KBps}
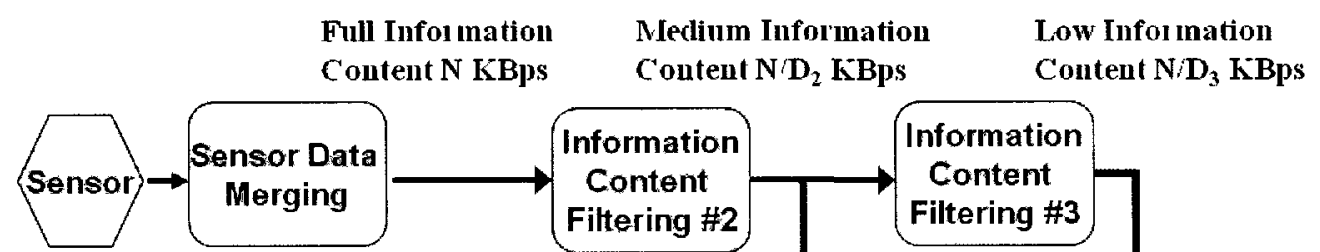

Total Buffer Size

$\mathbf{K}=\mathbf{B}_{2}+\mathbf{B}_{3}$

Buffer Size for

cached data $=B_{3}$

$=B_{2} * D_{2} D_{3}$

Buffer Size $B_{2}$
$K\left(1+D_{2} D_{3}\right)$
Data Stored:
$B_{2} * D_{2}$ : $N$ msec
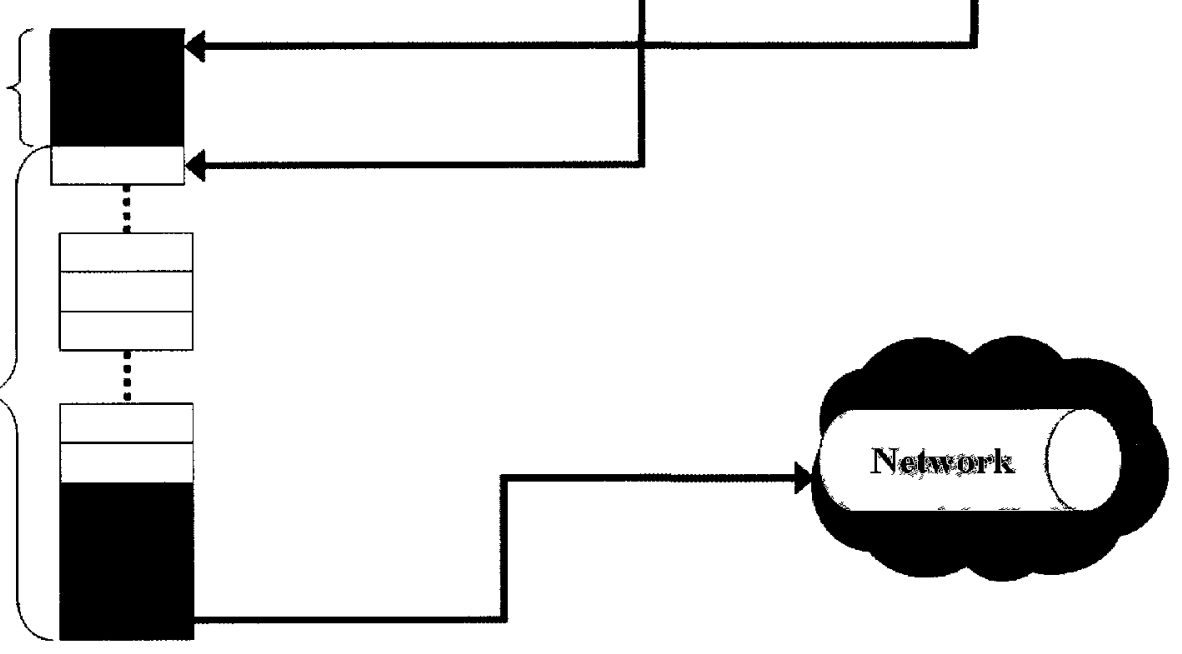

Figure 6.3: Adaptive information content and contiguous buffering RPM system with moderate network congestion.

In this case:

$$
\begin{aligned}
& B_{2}=\frac{K}{\left(1+D_{2} / D_{3}\right)} \\
& \text { and } B_{3}=B_{2} * D_{2} / D_{3}
\end{aligned}
$$


When network capacity drops further below N/D $\mathrm{DBps}$, the buffer $\mathrm{B}_{2}$ will over flow. In this case the data transmission will continue from Level \#3 with lower information content. Also all the buffering space previously used for storing data from Level \#2 will now be used for storing Level \#3 as shown in Figure 6.4.

In this case:

$$
\mathrm{B}_{3}=\mathrm{K}
$$

$$
\text { Heavy Network Congestion Network Data Rate }<\mathrm{N} / \mathrm{D}_{2} \text { KBps }
$$
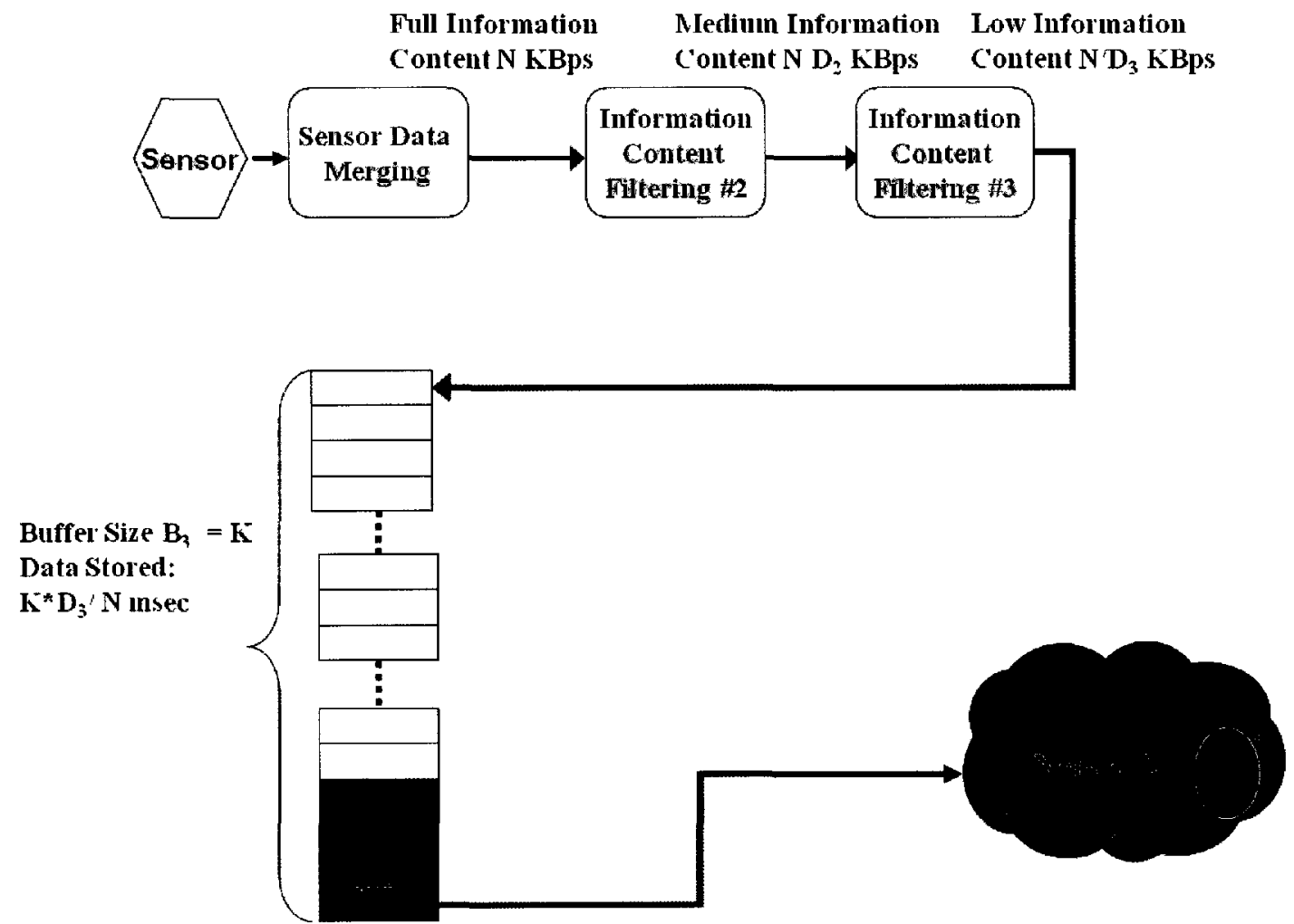

Figure 6.4: Adaptive information content and contiguous buffering RPM system with or heavy network congestion.

In the scenario shown in Figure 6.4, if the network capacity further drops below N/D 3 or connectivity are lost, buffer will overflow and all information content will be lost. 


\subsubsection{SI Evaluation for Contiguous Buffering Schemes}

The SI for the contiguous buffering scheme is given by

$$
S I=\sum_{i=1}^{3} S I_{i}
$$

where

$$
\begin{aligned}
& \mathrm{SI}_{1}=\frac{\mathrm{K} / \mathrm{N}}{\left(1+\frac{1}{\mathrm{D}_{2}}+1 / \mathrm{D}_{3}\right)} \\
& \mathrm{SI}_{2}=\frac{\mathrm{D}_{2} * \mathrm{~K} / \mathrm{N}}{\left(1+\mathrm{D}_{2} / \mathrm{D}_{3}\right)} \\
& \mathrm{SI}_{3}=\mathrm{D}_{3} * \mathrm{~K} / \mathrm{N}
\end{aligned}
$$

For plotting purpose it is assumed that $\mathrm{K} / \mathrm{N}=1$ for both the AICM and non-AICM method.

Using same 2 sets of values for $\mathrm{D}_{2}$ and $\mathrm{D}_{3}$ used in section 5.2.3,

For set 1

$$
D_{2}=2: 100 \text { and } D_{3}=2 * D_{2}
$$

and for set 2

$$
D_{2}=2: 100 \text { and } D_{3}=10^{*} D_{2} .
$$

the plots of SI for contiguous buffering scheme versus different values of $\mathrm{D}_{2}$ and $\mathrm{D}_{3}$ were obtained. SI for non-AICM RPM system $=1$ second in both cases.

Figure 6.5 shows the SI for $D_{2}$ varying from $2: 100$ and $D_{3}=2 * D_{2}$ (set 1 ). 


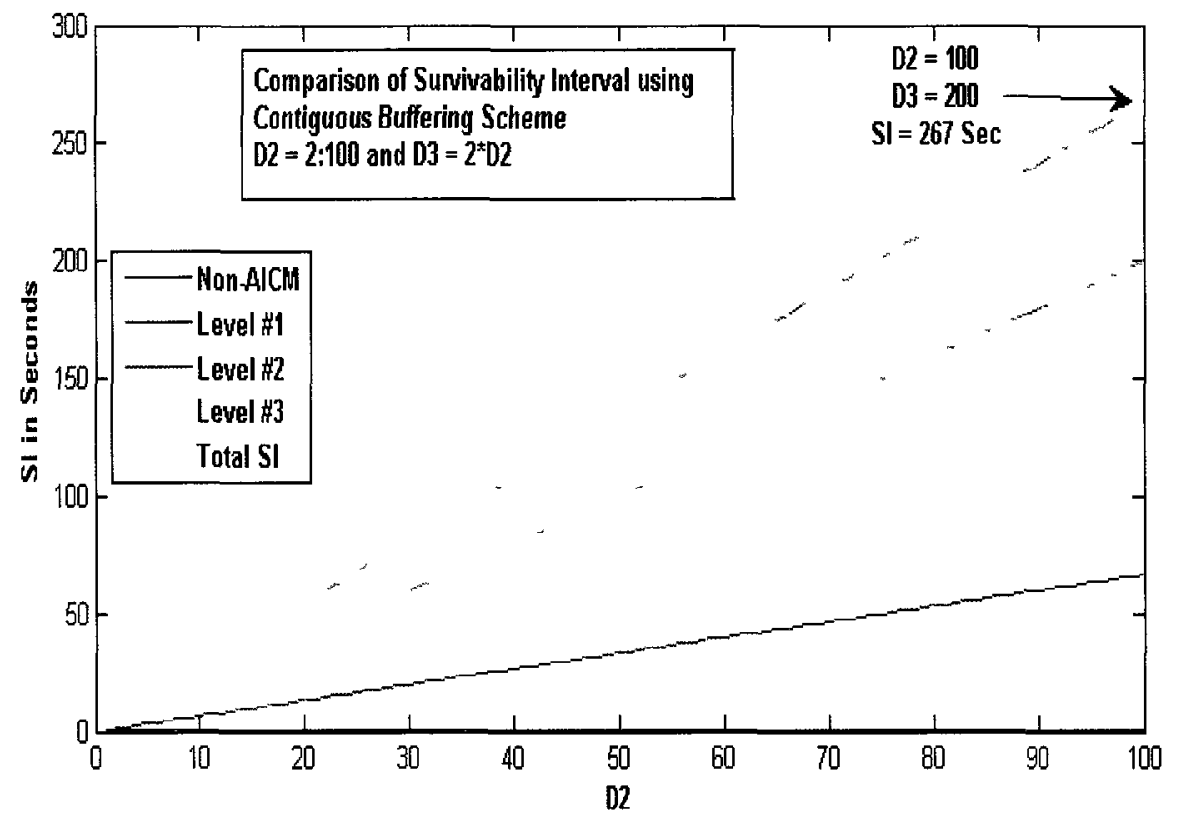

Figure 6.5: Comparison of Survivability Interval for Contiguous Buffering RPM system with and without AICM, $D_{2}=2: 100$ and $D_{3}=2 * D_{2}$

As seen from Figure 6.5 for data point when $\mathrm{D}_{2}=100$ and correspondingly $\mathrm{D}_{3}=200$ SI is equal to 267 seconds compared with 1 second for non-AICM method. Plot also indicates that as D2 increases (and correspondingly $\mathrm{D}_{3}$ ) SI of the AICM grows linearly.

Figure 6.6 shows similar plot of SI for $\mathrm{D}_{2}$ varying from $2: 100$ and $\mathrm{D}_{3}=10^{*} \mathrm{D}_{2}$ (set 2). As seen from Figure 6.6 for data point when $\mathrm{D}_{2}=100$ and correspondingly $\mathrm{D}_{3}=1000 \mathrm{SI}$ is equal to 1092 seconds compared with 1 second for non-AICM method.

Similar to results for static buffering scheme it is also clear from Figure 6.5 and 6.6 that most gain in survivability for 3-Level system is obtained when $D_{3}$ is much higher than D2. 


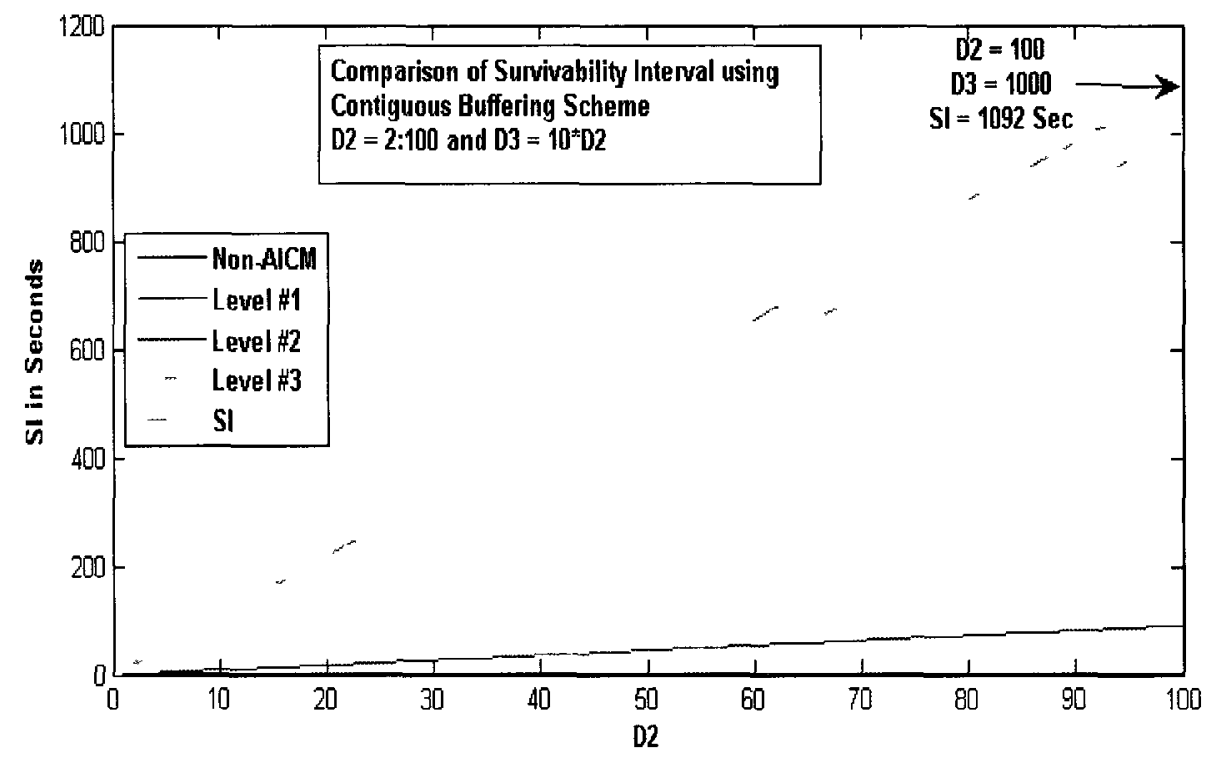

Figure 6.6: Comparison of Survivability Interval for Contiguous Buffering RPM system with and without $A I C M \quad D_{2}=2: 100$ and $D_{3}=10 * D 2$

\subsection{Comparison of the AICM system using Static vs.}

\section{Contiguous Buffering Scheme}

The Table 6.1 shows the summary of SI static buffering scheme and contiguous buffering scheme from Figures 5.5 and 5.6 and Figures 6.5 and 6.6 for the 2 sets of $\mathrm{D}_{2}$ and $\mathrm{D}_{3}$ used.

Table 6.1: Comparison of Static and Contiguous Buffering Scheme for $\left(\mathrm{D}_{\mathbf{2}}=100\right.$ and $\left.D_{3}=200\right)$ and $\left(D_{2}=100\right.$ and $\left.D_{3}=1000\right)$

\begin{tabular}{|c|c|c|}
\hline $\begin{array}{c}\text { Buffei ing Scheme } \\
\text { SI (in Sec) }\end{array}$ & Static Buffering & Contiguous Buffei ing \\
\hline$\left(D_{2}=100\right.$ and $\left.D_{3}=200\right)$ & 100.3 & $26^{7}$ \\
\hline$\left(D_{2}=100\right.$ and $\left.D_{3}=1000\right)$ & $36^{7}$ & 1092 \\
& & \\
\hline
\end{tabular}


From Table 6.1 it is clear that SI for contiguous buffering scheme provides the maximum survivability compared with static buffering scheme using the same total buffer size.

Figure 6.7(a) shows the plot of survivability interval for 3-Level static buffering RPM system using no AICM and system using AICM (given in Equation (5.1)) for $\mathrm{D}_{2}=2$ and $D_{3}=5$ while Figure 6.7(b) shows the equivalent plots for contiguous buffering scheme (using equations 6.12-6.14).

Figure 6.8 shows the equivalent plot of survivability interval for 3-Level RPM system with $D_{2}=10$ and $D_{3}=100$. Figure 6.9 shows the SI plot for 2-Level RPM system for $D_{2}=$ 10 and Figure 6.10 shows equivalent plot for $\mathrm{D} 2=100$.

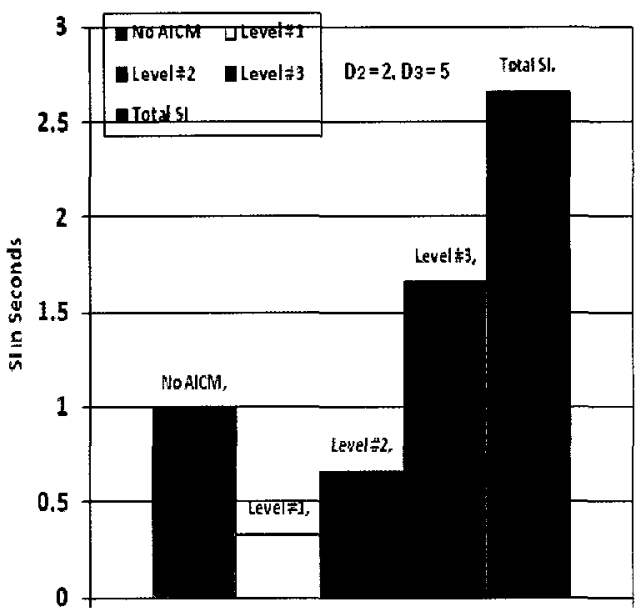

(a)

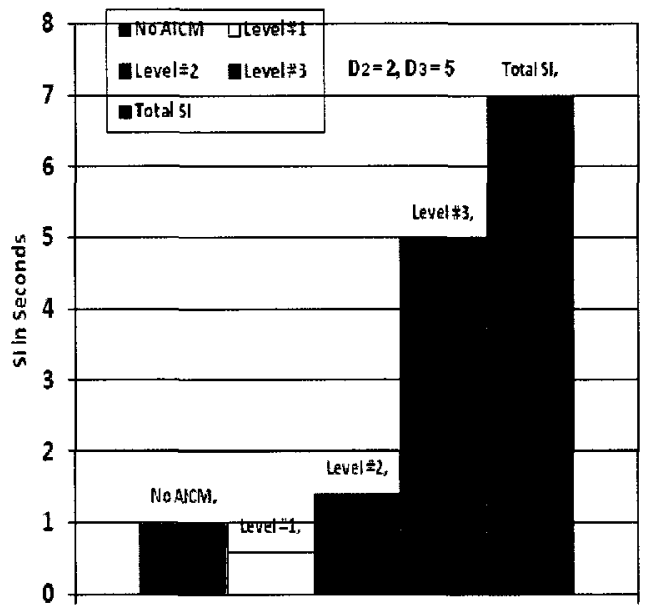

(b)

Figure 6.7: Comparison of Survivability Interval for Static (a) and Contiguous (b) Buffering RPM systems with no AICM and 3-Level AICM with D2=2 and D3=5. 

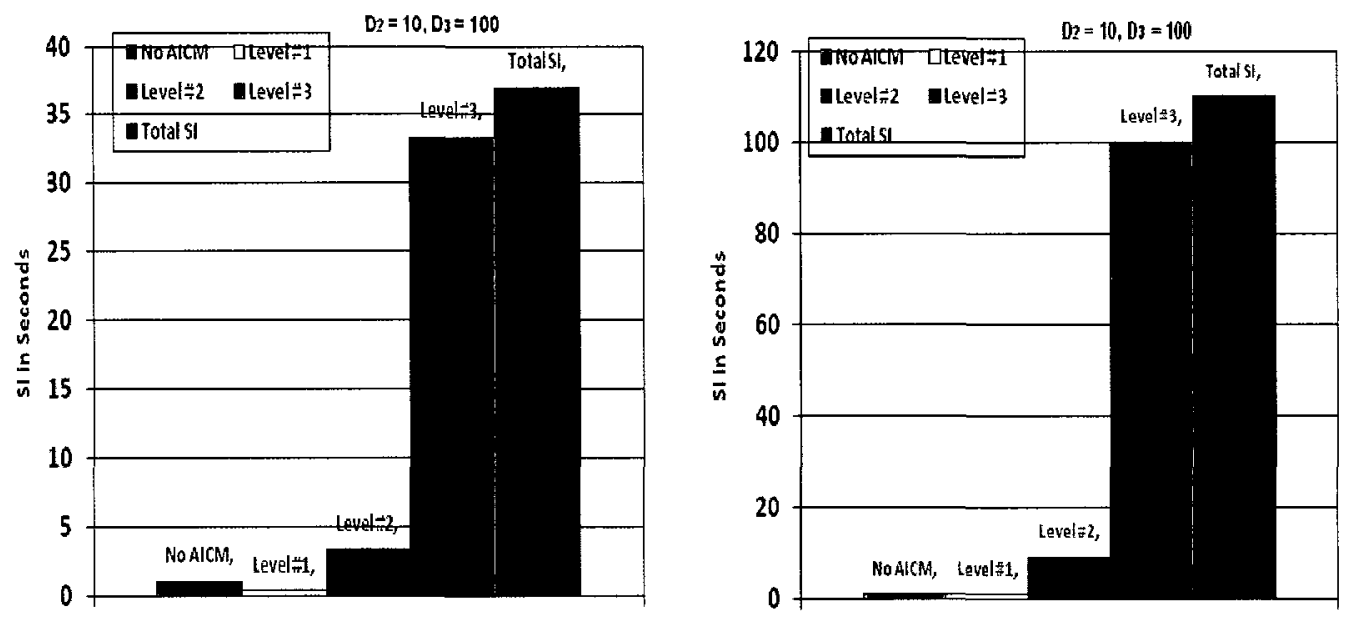

(b)

Figure 6.8: Comparison of Survivability Interval for Static (a) and Contiguous (b) Buffering RPM systems with no AICM and 3-Level AICM with D2=10 and D3=100.
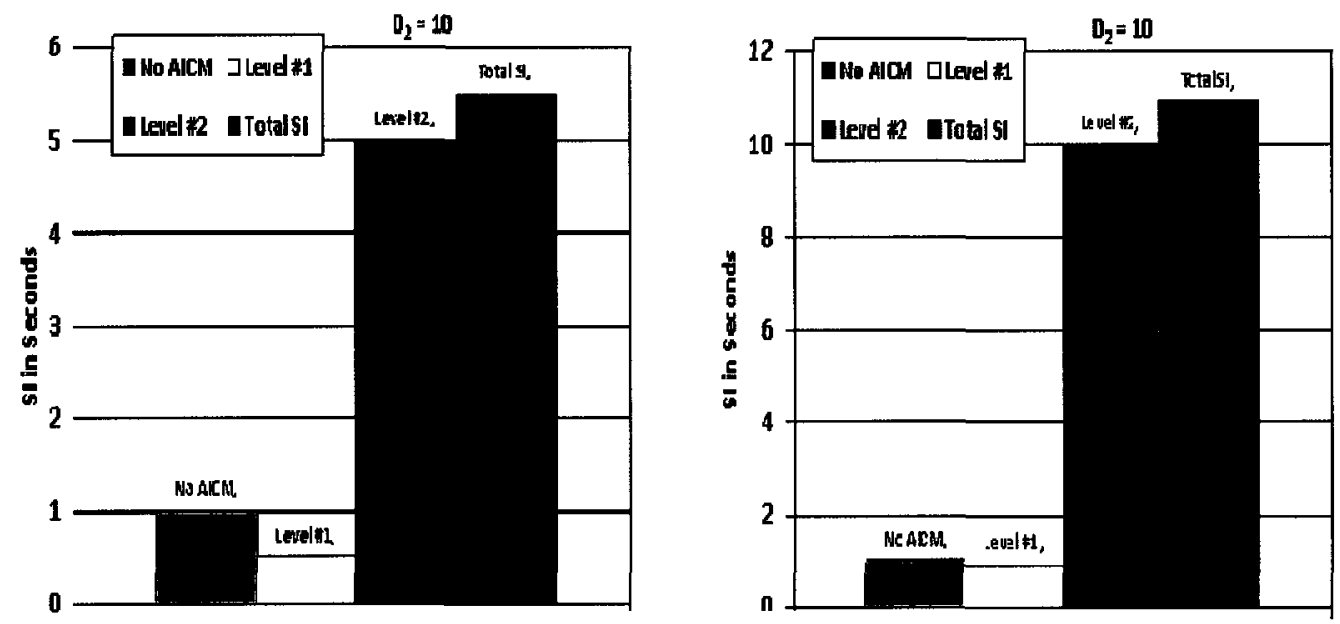

(b)

Figure 6.9: Comparison of Survivability Interval for Static (a) and Contiguous (b) Buffering RPM systems with no AICM and 2-Level AICM with D2=10. 

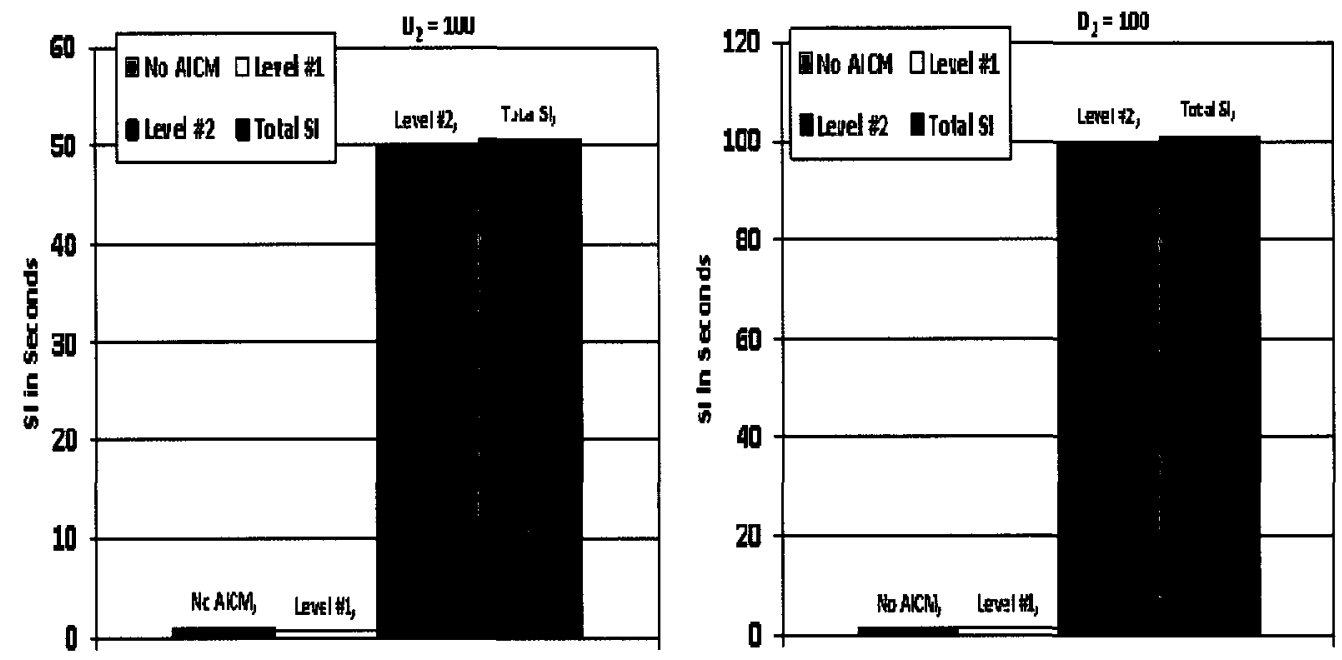

(b)

Figure 6.10: Comparison of Survivability Interval for Static (a) and Contiguous (b) Buffering RPM systems with no AICM and 2-Level AICM with D2=100.

As seen from Figures 6.8 to 6.10 it is clear that the AICM system using contiguous buffering scheme provides significantly higher survivability interval compared to the one using static buffering for both 2-Level and 3-Level systems. The result is extendable for levels $>3$.

These results clearly show that using contiguous buffering scheme for an AICM system will maximize the survivability of the RPM system under adverse network condition.

It is also evident from Figures 6.8 to 6.10 having higher number of Levels for static buffering scheme does not provide proportional gain in SI while contiguous buffering scheme does not suffer any reduction in efficiency. 


\subsubsection{Choice of Number of Levels for Static and Contiguous Buffering Schemes}

The choice of number of levels that may be used for the AICM method to obtain maximum SI is a design parameter and depends on values of $D_{i}$ available.

To illustrate how to obtain optimal number of levels for the AICM system it is assumed that the overall information content reduction possible for an AICM RPM system is given by " $D$ ". Such a system can have any number of intermediate levels ' $i$ '. This example compares SI for 2-Level system vs. 3-Level system. Assuming the following for both static and contiguous buffering schemes:

$$
\mathrm{D}=100 \text { and } \mathrm{K} / \mathrm{N}=1 \text { seconds }
$$

For 2-Level system

$$
\mathrm{D}_{2}=\mathrm{D}
$$

static buffering

$\mathrm{SI}_{\text {st }}(\mathrm{SI}$ for static buffering $)=1 / 2\left(1+\mathrm{D}_{2}\right)$

contiguous buffering

$$
\left.\mathrm{SI}_{\mathrm{co}} \text { (SI for contiguous buffering }\right)=\mathrm{D}_{2}+1 /\left(1+1 / \mathrm{D}_{2}\right)
$$

For 3-Level system

$$
\mathrm{D}_{3}=\mathrm{D} \text { and } \mathrm{D}_{2}=2: 5: 90
$$

static buffering

$$
\mathrm{SI}_{\mathrm{st}}=1 / 3\left(1+\mathrm{D}_{2}+\mathrm{D}_{3}\right)
$$

contiguous buffering

$$
\mathrm{SI}_{\mathrm{co}}=\mathrm{D}_{3}+\mathrm{D}_{2} /\left(1+\mathrm{D}_{2} / \mathrm{D}_{3}\right)+1 /\left(1+1 / \mathrm{D}_{2}+1 / \mathrm{D}_{3}\right)
$$


A plot of $\mathrm{SI}_{\mathrm{st}}$ for 2-Level and 3-Level system was obtained using equation (6.16) and (6.19) and shown in Figure 6.11. Similar plot of $\mathrm{SI}_{\mathrm{co}}$ for 2-Level and 3-Level system was obtained using equation (6.17) and (6.20) and shown in Figure 6.12.

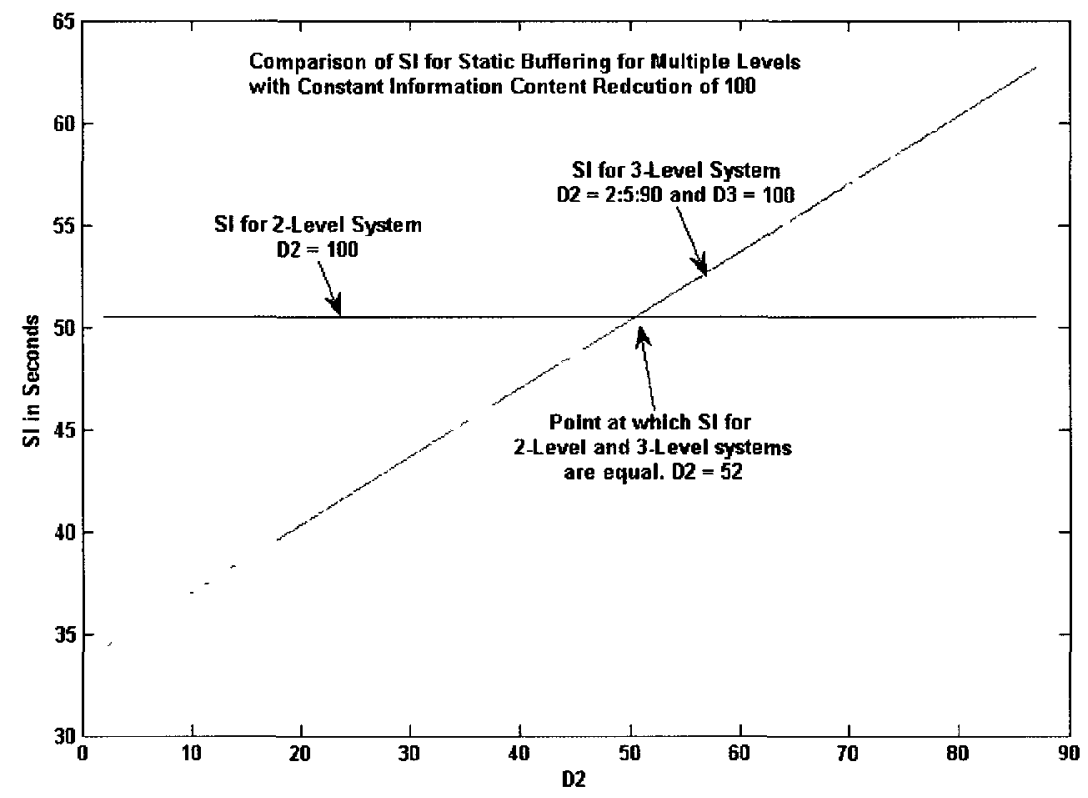

Figure 6.11: Comparison of $\mathrm{SI}_{\text {st }}$ for 2-Level and 3-Level static buffering system for same information content reduction $D=100$.

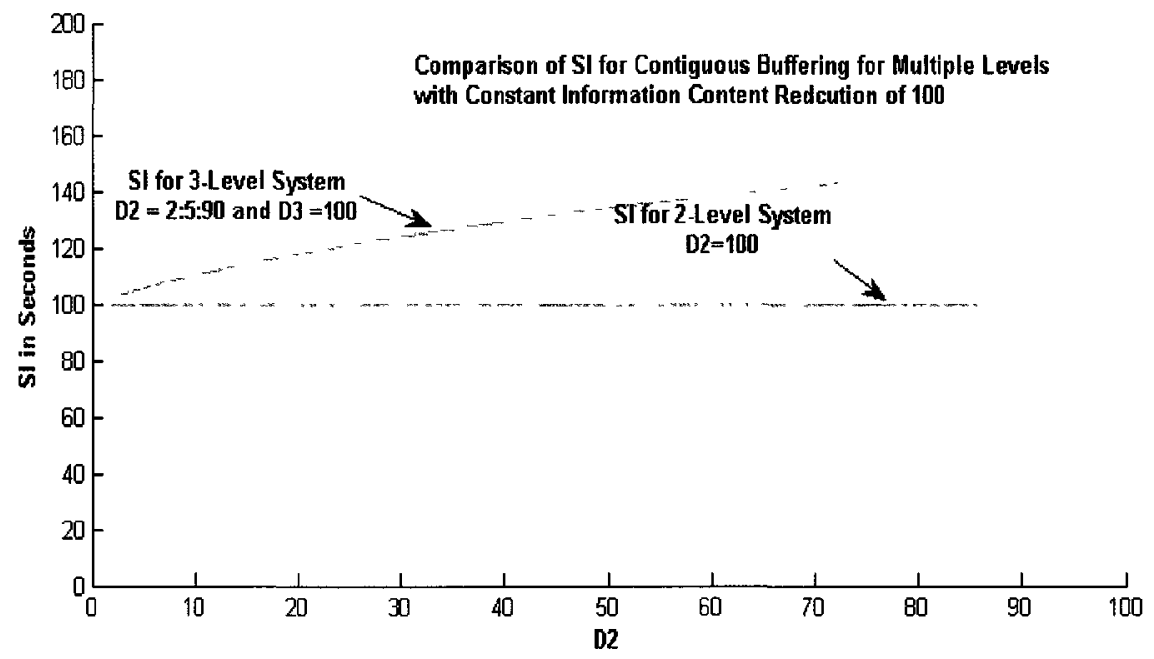

Figure 6.12: Comparison of $\mathrm{SI}_{\text {co }}$ for 2-Level and 3-Level contiguous buffering system for same information content reduction $D=100$. 
From Figure 6.11 it is clear that with static buffering scheme for a given information content reduction factor (100 in this case) up to certain values of $D_{2}$ (e.g. $D_{2}=52$ in this case) choosing 3-Level AICM system will provide reduced SI compared to a 2-Level AICM system.

Figure 6.12 shows that unlike static buffering scheme a 3-Level contiguous buffering system will always perform better than 2-Level contiguous buffering system. On the other hand for static buffering system having higher number of level is effective only after a certain threshold is reached. This clearly indicates the benefits of using contiguous buffering scheme for the AICM RPM system.

Similar analysis can be applied for choice of higher number of levels ( $L=4,5$ etc).

The choice of number of levels to be used for contiguous buffering system will depend on the sensors used and what content reduction factor $D_{1}$ can be achieved with the data while still maintaining the critical patient information.

For rest of the discussion in this chapter it is assumed that AICM RPM system is using contiguous buffering scheme.

\subsection{Performance of the AICM Method during Network}

\section{Congestion}

The amount of time " $\mathrm{T}_{1}$ " that a buffer in level ' $\mathrm{i}$ ' will take to overflow at different network capacities $C_{x}$, is given by:

$\mathrm{T}_{1}=\mathrm{K}_{1} /\left(\mathrm{R}_{1}-\mathrm{C}_{\mathrm{x}}\right)$

Where,

$\mathrm{K}_{1}=$ Buffer length for level ' $\mathrm{i}$ ' 


$$
\begin{aligned}
& R_{1}=\text { Data rate for level ' } i \text { ' }\left(N / D_{1}\right) \\
& C_{x}=\text { Network bandwidth }
\end{aligned}
$$

When

$$
\mathrm{N} / \mathrm{D}_{\mathrm{L}}>\mathrm{C}_{\mathrm{x}}
$$

all the buffers will overflow and all the patient data will be lost.

Total time, $T_{L}$, for buffers for L-levels to overflow is given by:

$$
T_{L}=\sum_{i=1}^{L} \frac{K_{l}}{\left(R_{i}-C_{x}\right)}
$$

where $\mathrm{R}_{1}=\mathrm{N} \mathrm{KBps}$

Defining

$$
\mathrm{N} / \mathrm{Cx}=\mathrm{M}
$$

and

$$
\mathrm{K} / \mathrm{N}=1
$$

Equation (6.22) for buffers to overflow gives

$$
\mathrm{M}>\mathrm{D}_{\mathrm{L}}
$$

Equation (6.23) can be written as:

$$
T_{L}=\sum_{i=1}^{m} \frac{\left(K_{i} * D_{i}\right) / N}{\left(\mathbf{1}-D_{\mathbf{i}} / M\right)}
$$

For contiguous buffering scheme using equation (6.2) $\mathrm{Ki}=\mathrm{Bi}$ and

$$
B i=\frac{K}{\left(1+\sum_{m=i+1}^{L} D_{i} / D_{m}\right)}
$$


When $\mathrm{C}_{\mathrm{x}}=0$ (Loss of connection) from equation (6.4) and (6.23) the time to overflow $\left(\mathrm{T}_{\mathrm{L}}\right)$ approaches SI.

$$
\mathrm{T}_{\mathrm{L}}=\mathrm{SI}
$$

Using equation (6.23) and (6.26) we can plot how the buffering system will behave as the network bandwidth reduces and buffers start to overflow. Figure 6.13 shows a plot of $T_{L}$ vs. $M / D_{L}$ for range of values of $M$ for 1 to 3 Level AICM system $(L=1,2,3)$ for contiguous buffering scheme.

A seen from the Figure 6.13 time it takes for buffer to overflow decreases rapidly when $M / D_{L}>>1$. Also seen from Figure $6.13(B)$ and $(C)$ when $M / D_{L}>2$ the overflow time come is very close to the SI (when connection is lost, $\mathrm{C}_{\mathrm{x}}=0$ ).

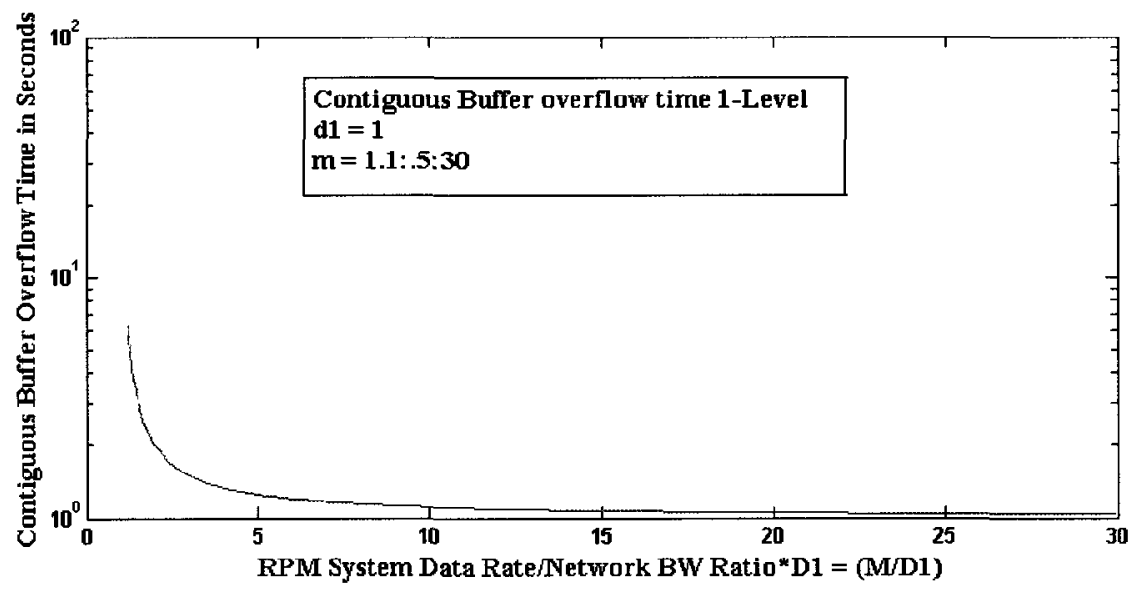

(A) 


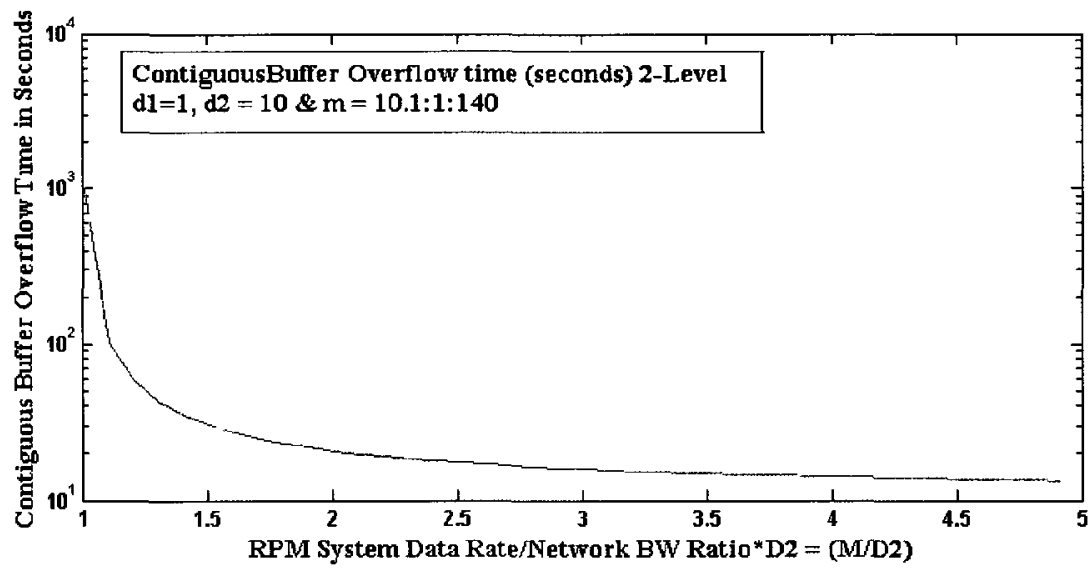

(B)

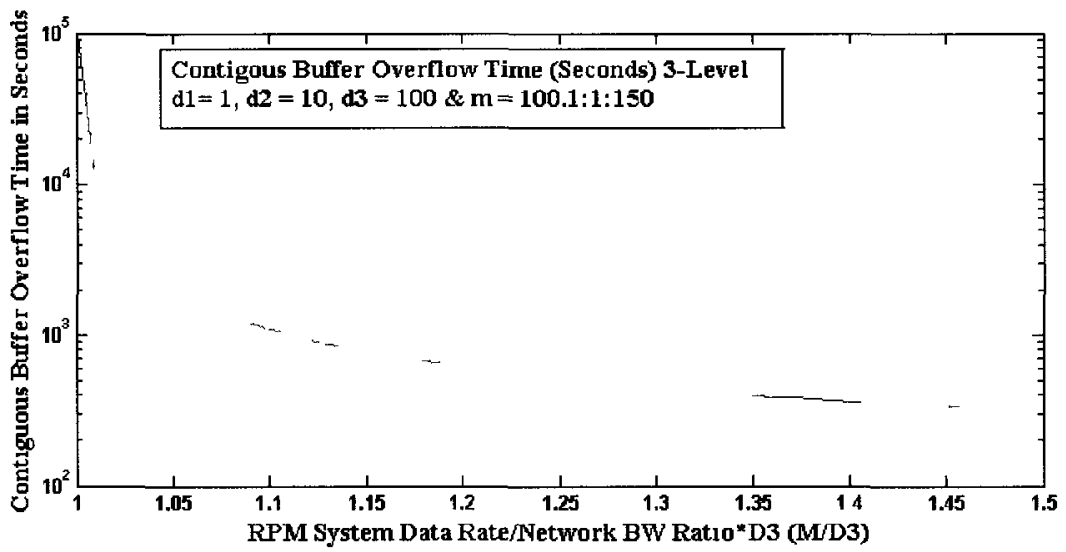

(C)

Figure 6.13: Plot of adaptive information content management RPM system under different network congestion contiguous buffering scheme.

Figure 6.14 shows 3-Level system overflow time for Level-2, Level-3 and Total with $\mathrm{D}_{2}$ $=50$ and $\mathrm{D}_{3}=100$. Figure 6.15 shows similar plot with $\mathrm{D}_{2}=5$ and $\mathrm{D}_{3}=10$. 


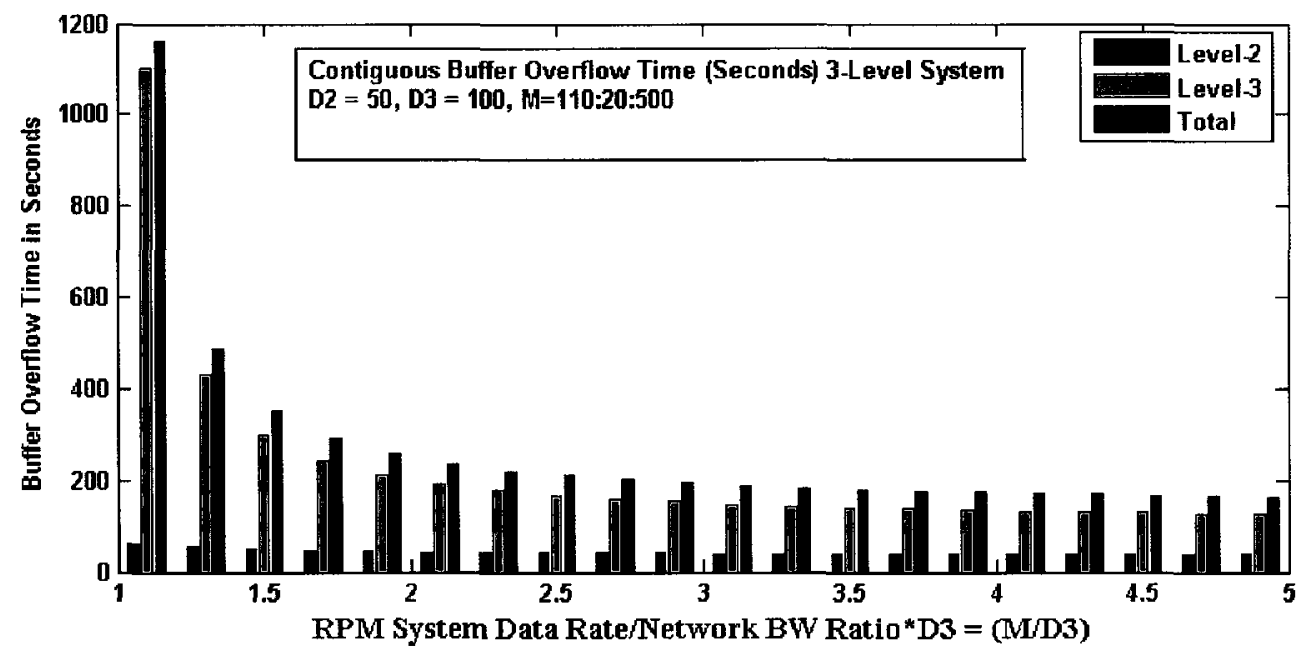

Figure 6.14: Overflow time in seconds for 3-Level AICM system with $D_{2}=50$ and $D_{3}$ $=100$.

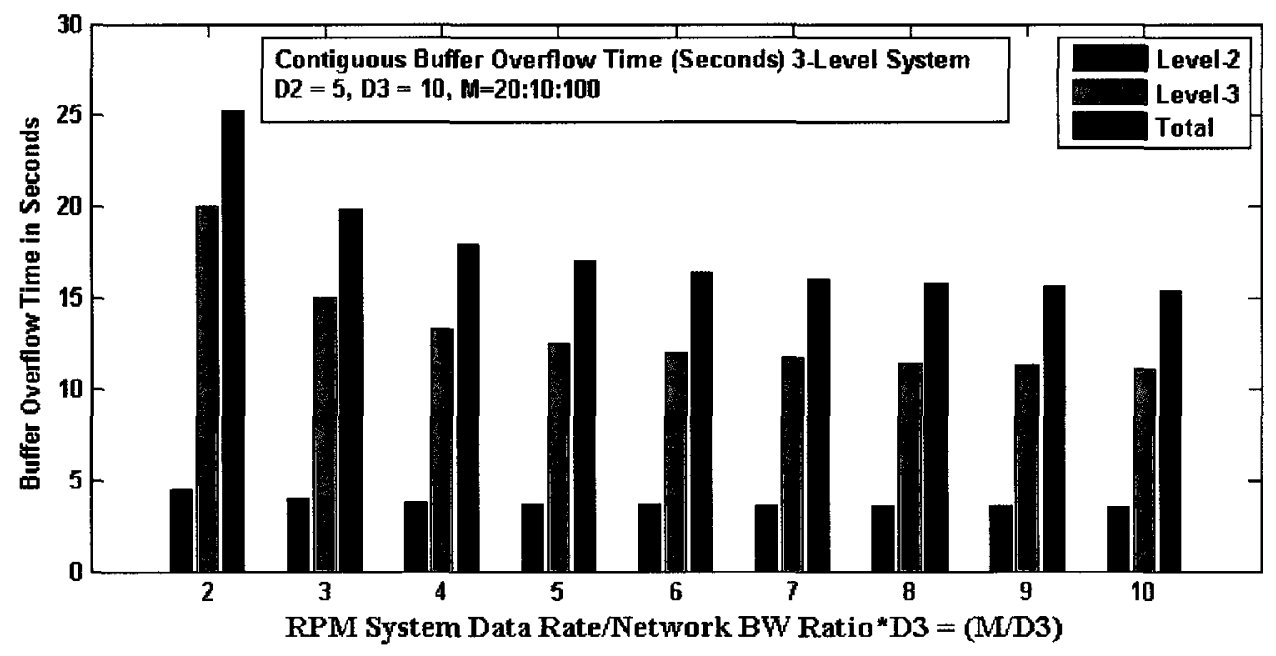

Figure 6.15: Overflow time in seconds for 3-Level AICM system with $D_{2}=5$ and $D_{3}$ $=10$.

These plots can be used to design the number of levels and corresponding information content reduction factor $D_{i}$ needed to achieve certain SI and overflow time. Given the $D_{i}$ and number of levels $\mathrm{L}$ in the system they can be used to obtain SI and overflow times for the overall system. 


\subsection{Summary}

A contiguous buffering scheme for storing multi-level information content provides significantly superior performance in terms of SI compared to a static buffering scheme presented in Chapter 5, which allocates fixed number of buffers for each information content level. The number of levels that can be used in an RPM system will depend on many different design requirements and criteria's and not only on overall SI. The important criteria's that will be considered are e.g. critical real-time sensor data, network capacity and congestion pattern, reliability of the network, available buffer size, the patient condition etc. Using the equations derived in this section the SI performance of the system can be computed for given information content reduction possible with the RPM system design. The SI can be used to generate alarms if network connectivity loss interval exceeds SI. 


\section{Chapter 7: Implementation of an Bed Occupancy RPM System Using Pressure Sensitive Mat}

This chapter presents the Bed Occupancy RPM system implemented in this thesis to illustrate how the concepts developed in Chapter 4, 5 and 6 can be used for real life implementation.

The sleep pattern monitoring is critical for many medical conditions, also changes in sleep patterns are an early sign that an illness may be accelerating, people with dementia have difficulty maintaining regular sleep pattern at night and may show increasing sleepiness during the day [46], [49], [50]. Currently sleep pattern is monitored using sleep diary or verbal information provided by the patient. Such sleep data can be incomplete and may not be accurate. An objective sleep pattern monitoring can be provided by nonintrusively monitoring the bed occupancy.

The RPM system for bed occupancy was implemented as a part of a collaborative study between Faculty of Nursing at University of Toronto, Ottawa-Carleton Institute for Biomedical Engineering at Carleton University and Dept of Geography \& Environmental Studies at Wilfrid Laurier University Waterloo. The project was undertaken to investigate the role telemonitoring technology can play in supporting the clinical decision making of 
home care clinicians ${ }^{1}$. The study investigated the impact of providing clinicians with access to the following data:

- Sleep quality and the ability to transfer in and out of bed collected using a pressure sensitive mattress.

- Mobility patterns collected via a GPS enabled device such as a Blackberry.

These data are expected to be used to augment functional health assessments, support patient education and enable clinicians to more effectively and efficiently monitor client recovery.

The overall project objective was to develop and integrate a non-intrusive monitoring system to support clinicians in their decision-making and monitoring of home care clients' health in and out of the home. The project's goals are to improve the efficiency and safety of home care services, reduce the demand on scarce and expensive human resources, and improve the safety and quality of home care services.

The system uses custom built software on the BlackBerry that automatically acquires compresses and securely transmits data from the on-board GPS/accelerometer and from pressure sensitive mat to a central node. Data processing algorithms are then used to automatically detect a wide range of patient activities including sleep duration, bed exits, out-of-home activities, exercise and in-home movement patterns [5], [6], [7], [8], [9].

The project was divided in 2 components; the bed entry/exit part was implemented at Carleton University while GPS mobility data component was implemented at Wilfrid Laurier University.

\footnotetext{
${ }^{1}$ This research is supported by a grant from the Research (CIHR) and Natural Sciences and Humanities Research Council (NSERC).
} 


\subsection{Bed Occupancy RPM System}

The implemented Bed Occupancy RPM system is designed measure to patient activity in bed especially bed entry and exit timings. For the implementation of Bed Occupancy RPM system a pressure sensitive mat by S4 Sensors Controls Inc called Bed Occupancy Sensor $\left(\mathrm{BOS}^{\mathrm{TM}}\right)$ was used. The BOS ${ }^{\mathrm{TM}}$ is a waterproof sensor pad that is placed under a client's mattress in the patient's home. This allows doctors to remotely monitor sleeping patterns of their patients. The Figure 7.1 shows the block diagram of the implemented RPM system. The bed occupancy sensor based RPM system was implemented as a part of this thesis. The external web server used was implemented by Wilfrid Laurier team.

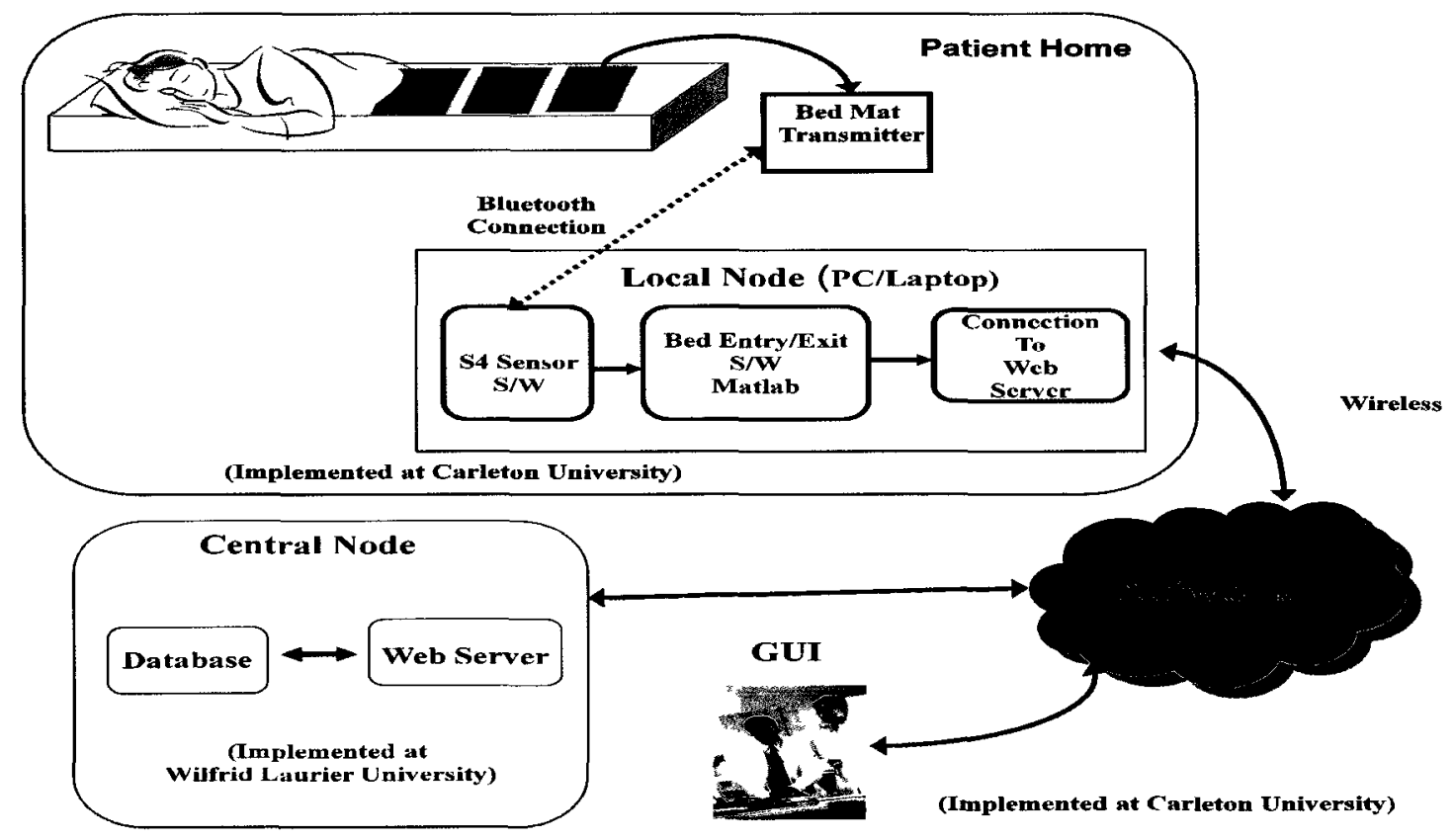

Figure 7.1: Block diagram of Bed Entry/Exit RPM System.

The Bed entry/exit detection software in the local node and the GUI was implemented as a part of this thesis using Matlab. 


\subsubsection{Pressure Sensitive Mat}

The pressure sensitive mat BOS $^{\mathrm{TM}}$ translates pressure exerted on embedded sensors in to electrical signal. This signal can then be used to detect presence and/or absence of occupant in the bed. The BOSTM consists of 24 sensors arranged as an array of $8 \times 3$ sensors. The size of the BOS ${ }^{\mathrm{TM}}$ mat is $24 \mathrm{~cm}$ by $90 \mathrm{~cm}$, with sensor elements spaced 10 $\mathrm{cm}$ apart. The pressure sensor uses a light emitting diode (LED) with two fiber optic wires for transmitting and receiving signal. The transmit fiber allows light to propagate through it from the LED into the sensor cavity. The receive fiber measures the intensity of the backscattered light which is converted in to the force applied on the sensor. The measurements that are being recorded by the sensors are relative measures of force being applied to the mat.

The sensors were sampled with 12 bit precision at $10 \mathrm{~Hz}$. Samples from each sensor are forwarded over the network by S4 Sensors Inc. firmware as three semi-redundant 2byte words, resulting in a data rate of $\sim 10 \mathrm{KBps}$ (for 24 sensor Bed Mat).

The output from mat is sent to the local node (a PC/Laptop in this case) via a Bluetooth connection where the software provided by S4 Sensors receives the pressure data and stores it in a Comma Separated Value (.CSV) file on the local node. This file is Microsoft Excel compatible. The data is stored in separate file of 4 hours (manually adjustable) intervals. Every 4 hours a new data file is created and old file closed. All the files have unique file name. The data stored contains time stamps and pressure value for each sensor.

The mat setup for bed entry/exit can use one pressure mat or six pressure mats. While one pressure mat is sufficient to measure bed entry/exit data, using six mats allows 
additional data besides bed entry/exit to be collected. This additional data allows measurement of length of time it took for a client to exit the bed, how symmetrical an exit was, and whether or not they used their hands to get out of bed. Recording changes in bed entry and exit patterns may signify changes in the physiological condition of the client. Figure 7.2 shows how one sensor and six sensor mat setup is used.

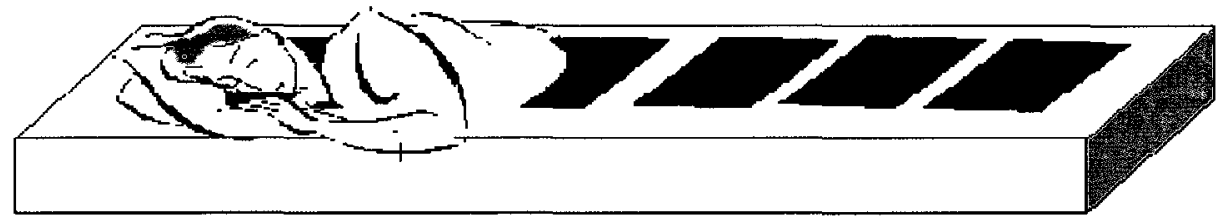

(A) - Six Pressure Sensor Set-Up

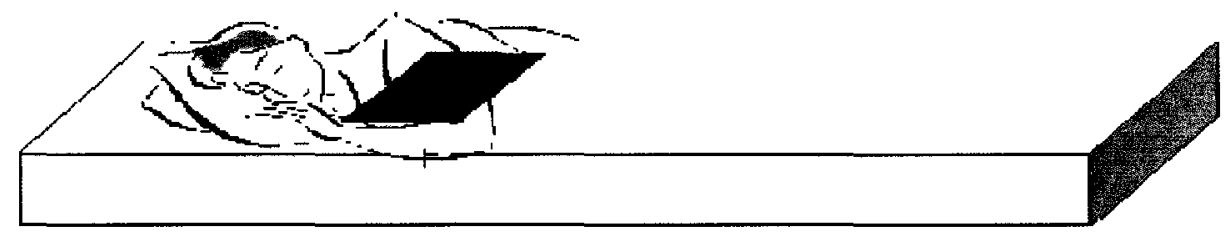

(B) - One Pressure Sensor Set-Up

Figure 7.2: Different pressure mat set up used for bed entry/exit RPM system.

\subsubsection{Usability Study for GUI Design}

GUI design is an important aspect of the RPM system for users of the system (healthcare providers). Presentation of the monitored data must be intuitive and easily navigatable for user acceptance. It should help with user tasks and not cause information overload.

One goal of the study was to determine the most appropriate GUI features of the monitoring system for data presentation. This was achieved by conducting a usability test and qualitative analysis of the GUI prototype. The usability study was undertaken early in the GUI design cycle to solicit feedback from the healthcare providers regarding GUI requirements. So the feedback can then be incorporated into subsequent GUI designs. 
The usability study was conducted by Anneliis Tosine and me from Carleton University and research teams from Faculty of Nursing at University of Toronto, and Dept of Geography \& Environmental Studies at Wilfrid Laurier University Waterloo.

The study research team used a human-centered design approach and prepared low fidelity prototypes of GUI for usability test [127]. Two possible versions (called Version A and Version B in our study) of a prototype GUI that encompasses the data outputs of the monitoring technologies at Carleton (pressure sensor mat) and Wilfrid Laurier (GPS using Blackberry) universities were designed by research team consisting of myself and Anneliis Tosine at Carleton University and a researchers from Wilfrid Laurier university. Each GUI version consisted of three levels of data in a 'dig-down' format. Usability goals/ requirements and evaluation objectives were also outlined in this step. Figure 7.3 and 7.4 shows the homepage of the GUI for version A and B.

A pseudo patient with a case history was created for the demo. 
Ranger, Joe

Welcome Smith, John Today is Tuesday November 172009

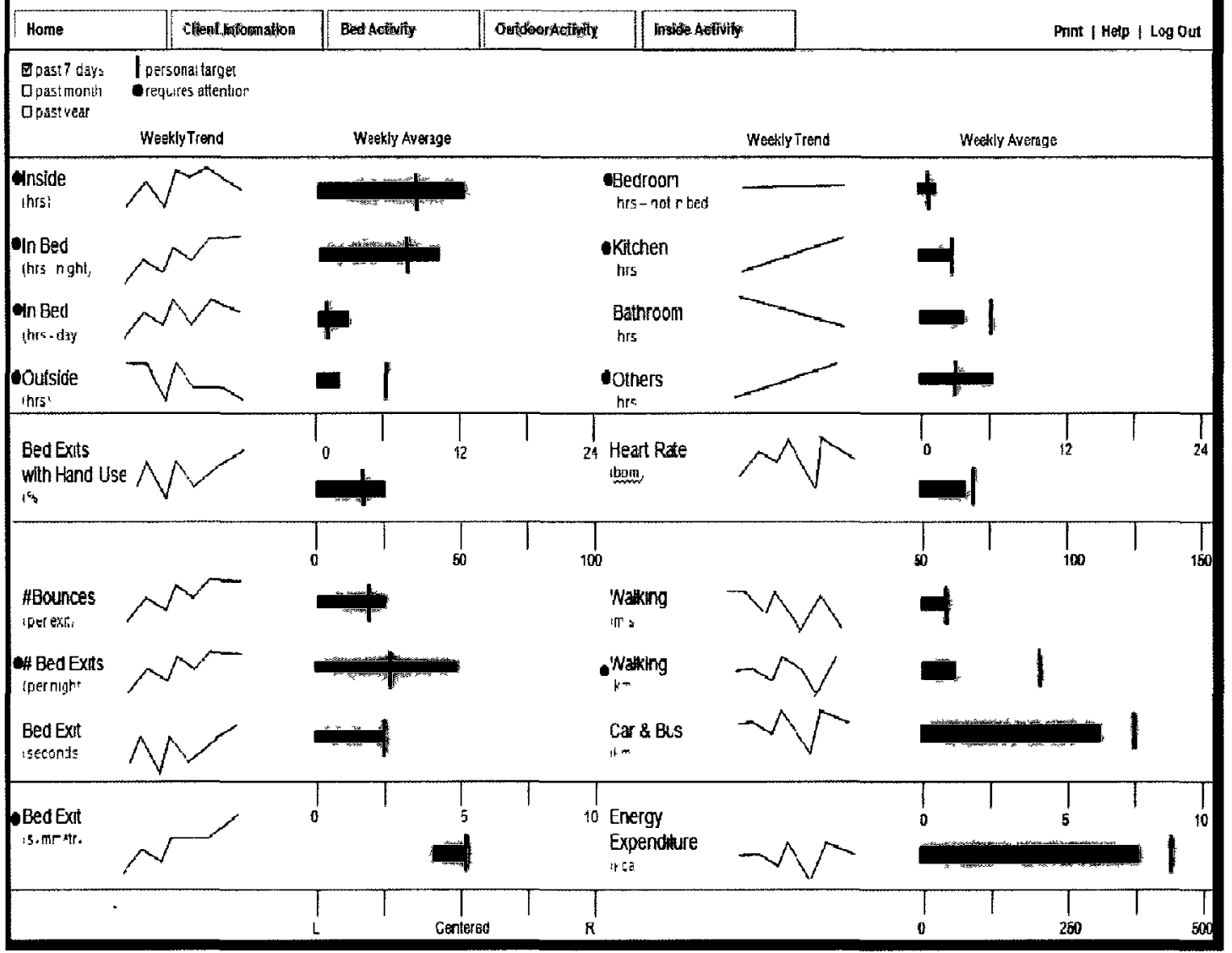

Figure 7.3: Legend location and size for the homepage on Version A. 
Ranger, Joe

November 17,2009

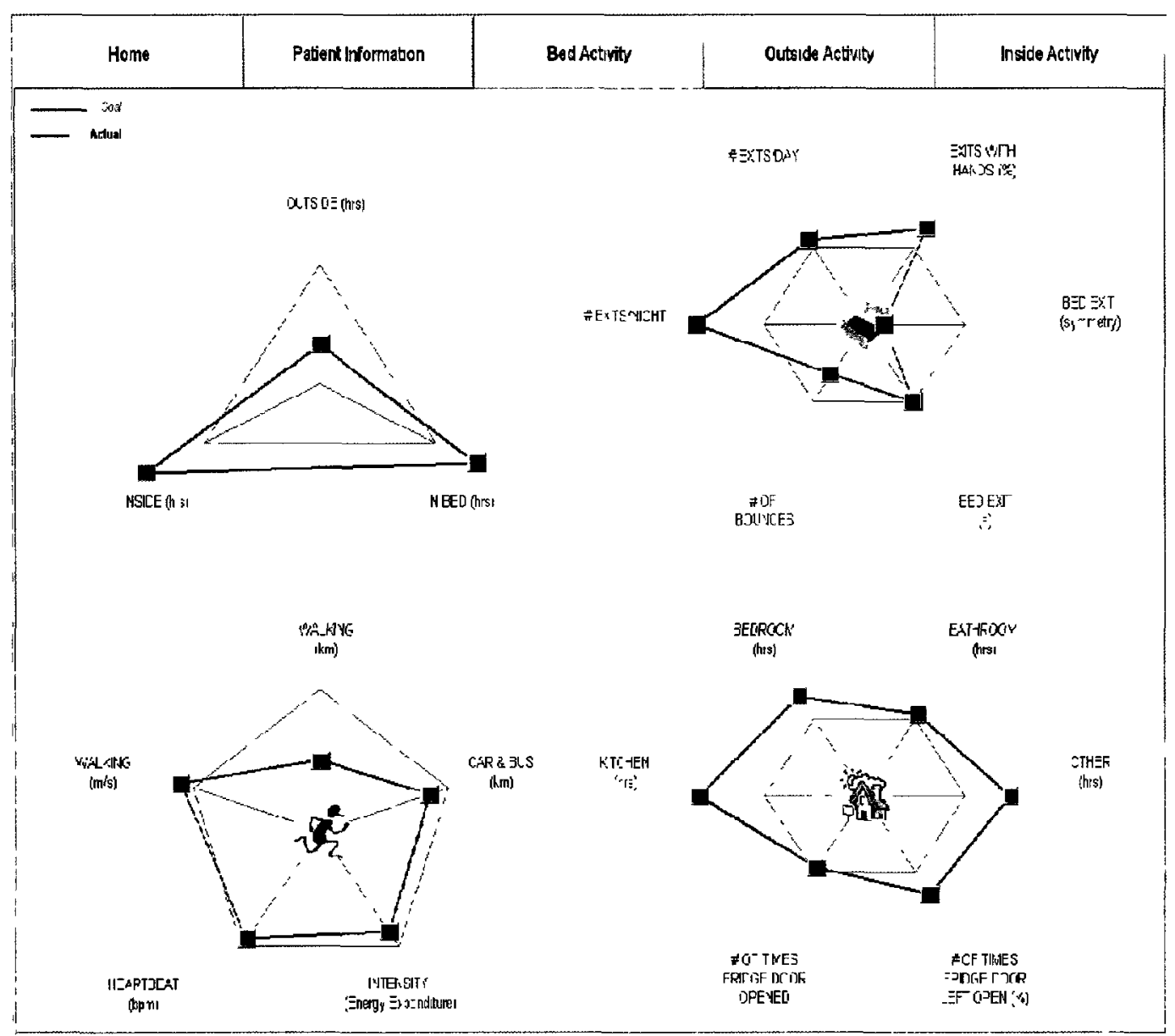

Figure 7.4: Legend location and size for the homepage on Version $B$

The Figure 7.5 and 7.6 shows the GUI page for the bed activity tab version A and B that can be accessed from home page shown in Figure 7.3 and 7.4.

The usability trial was conducted for three types of user groups that the system was designed for, home care nurses, occupational therapists, and case managers. A case description was created for each clinician type asking the participants to complete certain number of tasks while using the GUIs. The study cases were drawn from cases commonly encountered during home visits. 


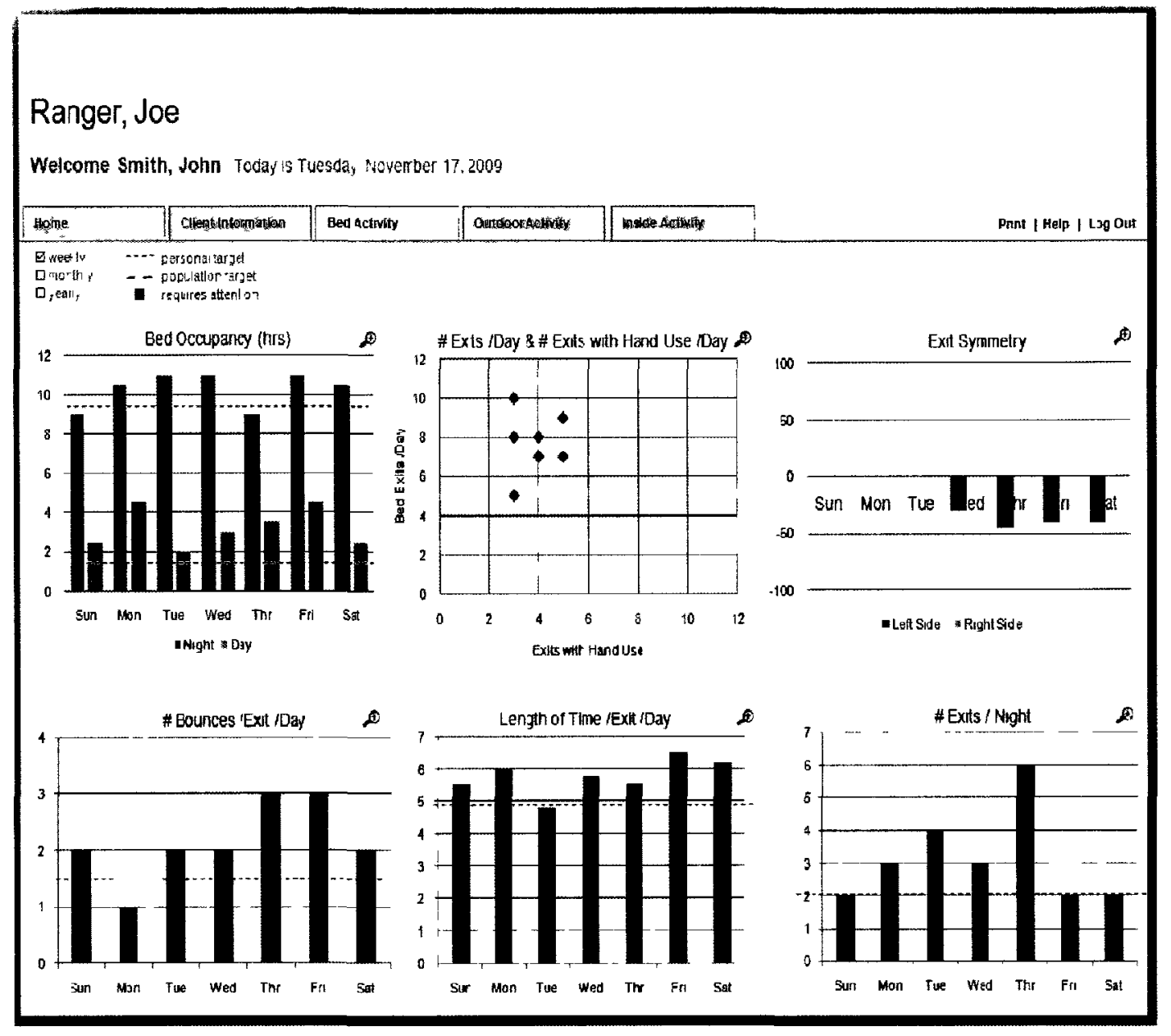

Figure 7.5: Bed activity tabs in Version A, Level-2.

For each usability test, the set-up involved a video recording of the computer screen to document mouse movement and better understand the thought process and interface interaction of the participant - as well as a back-up audio recording. The evaluation setup is shown in Figure 7.7.

The audio recordings were transcribed and used together with the video recordings. Through iterative passes of eight participant audio transcriptions and videos, similar parameters surfaced. These parameters were grouped into two categories; issues pertaining to detailed design and those related to conceptual design. 


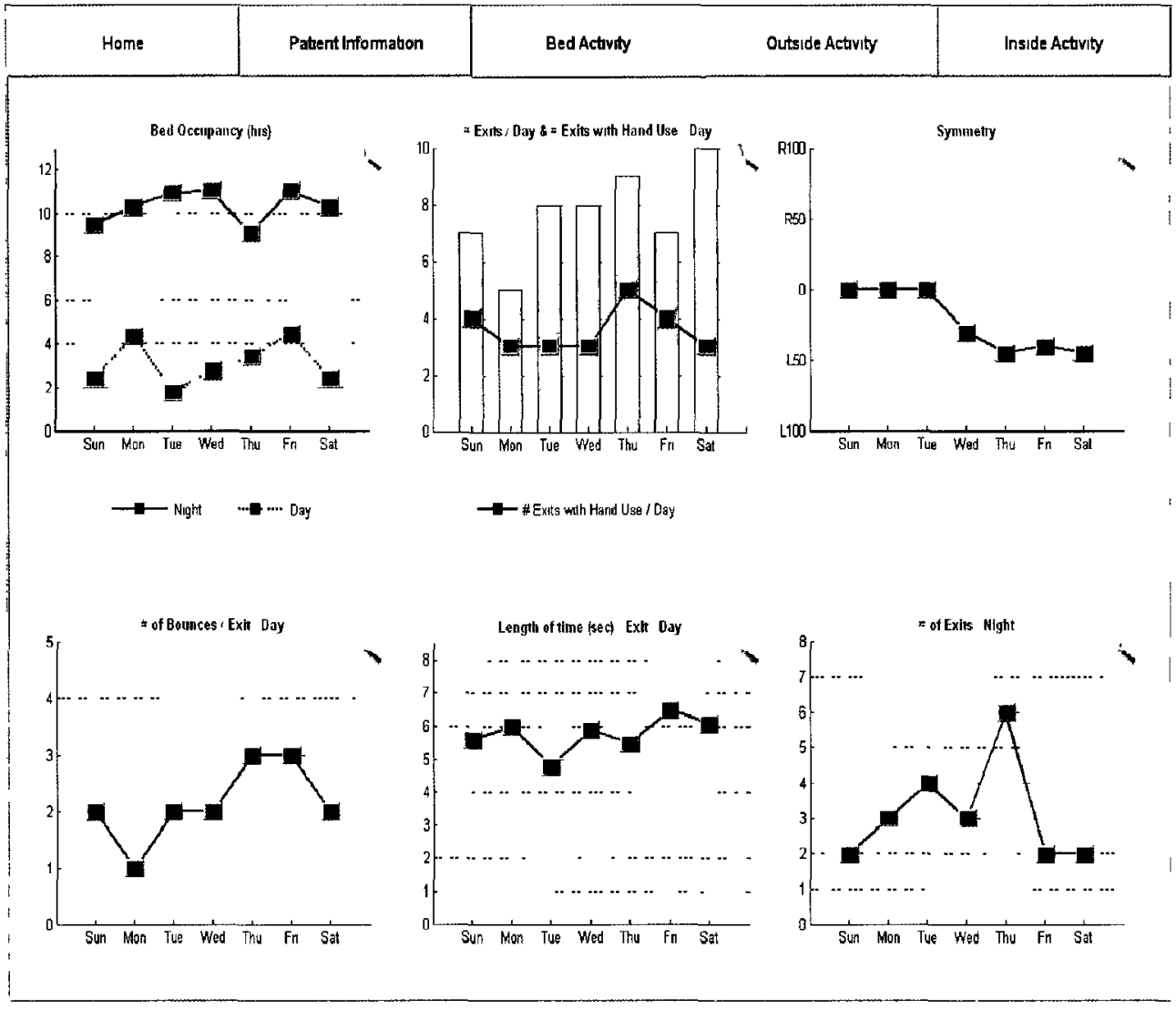

Figure 7.6: Bed activity tab in Version B, Level-2.

Since this was a formative evaluation, additional evaluations can be repeated to reveal how changes made to the prototype based on this usability study affects the system's usability. This iterative method helps to integrate the feedback from the users in the design and development process of the GUI in order to continuously improve the information system, help gauge/measure any developmental progress, and assure a higher acceptance rate for its integration into the home care practice [8], [9].

Using the results of the usability study a GUI using Matlab specifically intended for use with bed occupancy RPM system was implemented as a part of this thesis. 


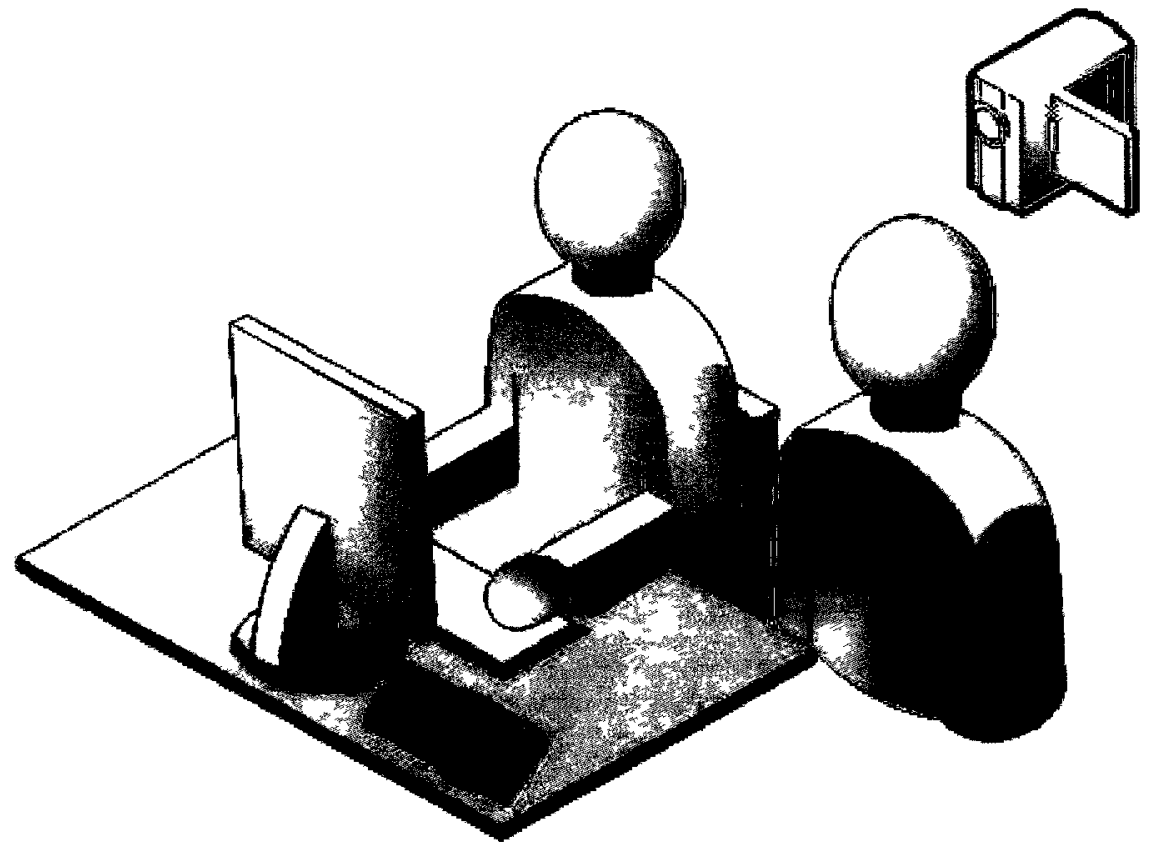

Figure 7.7: GUI evaluation set-up

\subsubsection{Local Node}

The data from mat is received by the local node. The mat transmits the data using Bluetooth technology. The local node in this case is either a PC or laptop. The local node processes the received data from sensors to determine the bed entry and exit times. The data processing software to extract bed entry/exit time was implemented using Matlab. The local node has the Matlab installed on it. It is capable of detecting bed occupancy of any duration. The software works with data from both one mat and six mat configurations. The data processing software runs automatically every day to obtain bed entry/exit times. The result file can be send to central node automatically using a Blackberry. This bed occupancy data can then be plotted to understand the daily, weekly and monthly trends. 


\subsubsection{Trial of Bed Occupancy RPM System}

An integrated trial of the Bed Occupancy RPM system from Carleton University and GPS mobility pattern system developed by Wilfrid Laurier University was conducted in the $1^{\text {st }}$ week of November 2010 . Pressure sensor mat was installed in participant's bed and participant also carried Blackberry for monitoring mobility (movement) outside home. A laptop with Matlab installed was used to collect the data from pressure mat via Bluetooth connection. The participant went about their daily activities as usual. The trial was conducted for a period of 10 days. The trial was successful with no issues found with use of mat in participant's home.

The collected mat data from the trial was processed and daily, weekly and monthly trends were obtained. The designed GUI was used to show how the processed data can be used by healthcare providers to monitor the patient, understand the patient's progress against the set targets and adjust the treatment if needed. The patient name and information used in the GUI is fictitious and for demonstration purpose only and is not related to trial participant for privacy purposes.

Figure 7.8 shows homepage of the bed occupancy RPM system GUI. It shows the details of the patient, provides choice of displaying daily/weekly/monthly data, allows the healthcare provider to change dates/week/months to review history. The bed occupancy data is shown as bars with a threshold line indicating to the healthcare provider how well the patient is doing against the target. Threshold can be set by healthcare provider. There is also space available for healthcare providers to write down the note. 

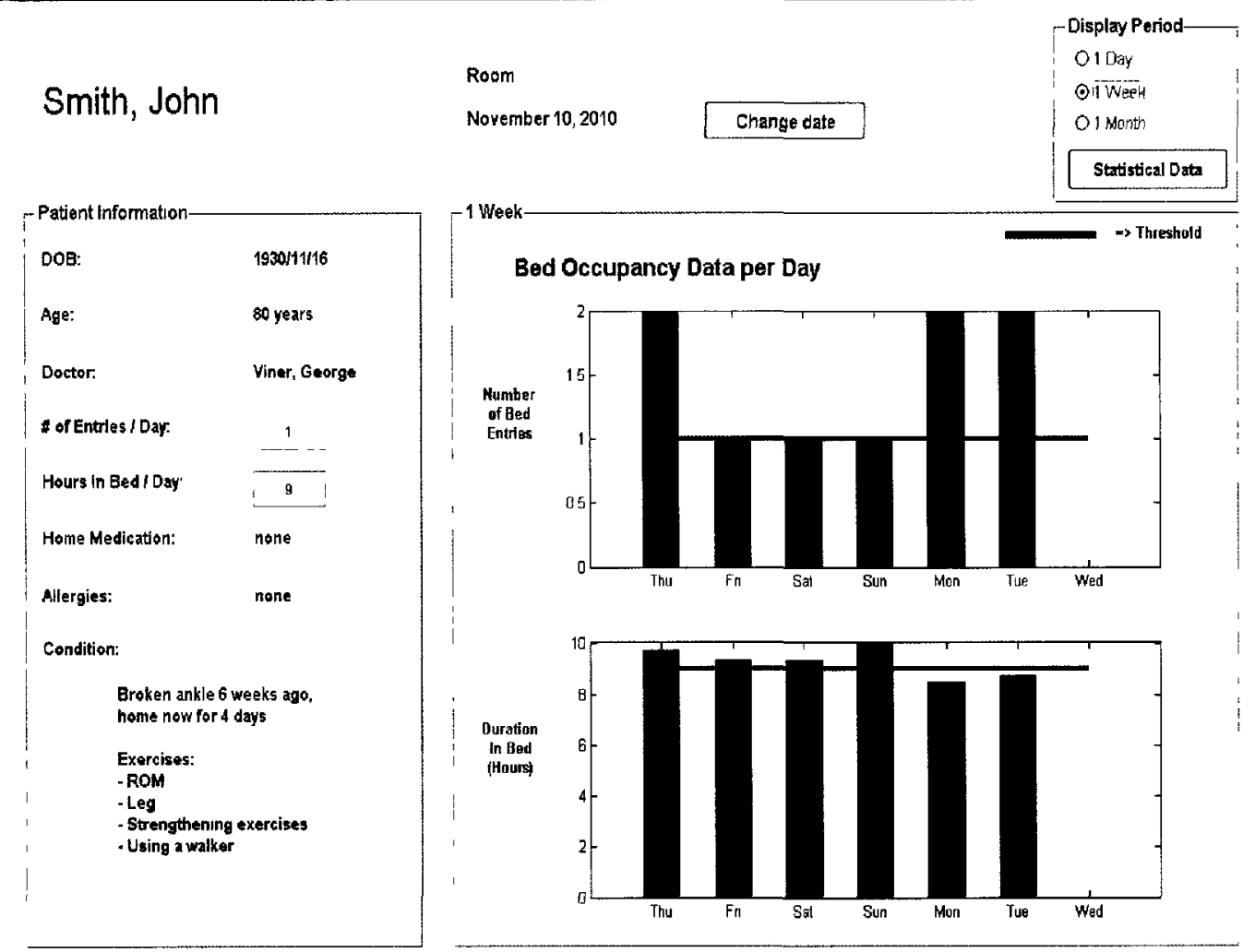

Figure 7.8: GUI for bed entry/exit RPM system, showing weekly bed occupancy data.

Figure 7.9 shows the monthly view from the GUI. There is a button on the GUI homepage to look at the statistics of the bed occupancy data. The "Statistical Data" button on the GUI can be used to view various statistical data like avg. wake-up time, avg. length of naps, avg. bed entry time and avg. no of bed exits at night. 


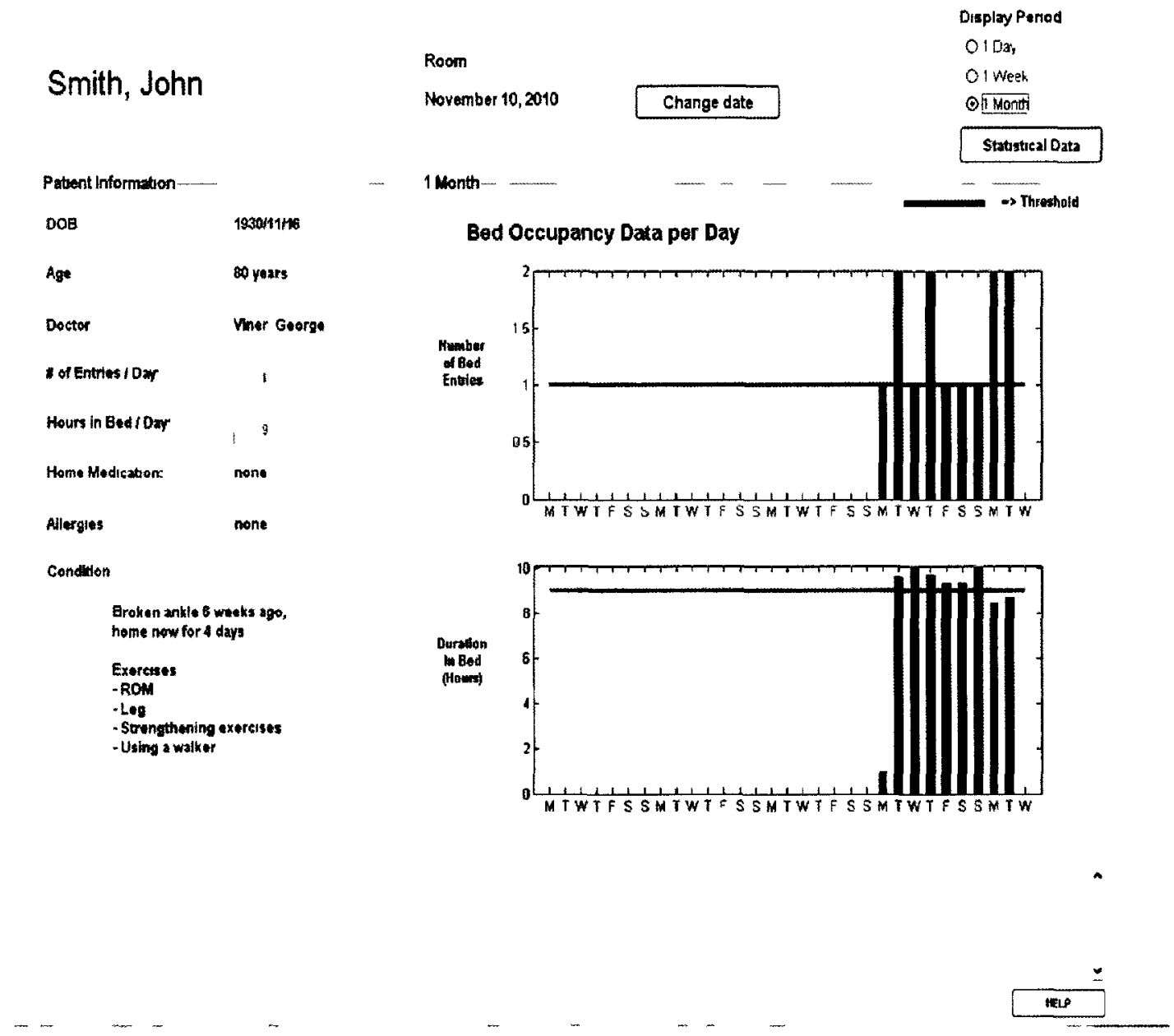

Figure 7.9: GUI for bed occupancy RPM system, showing monthly view of bed occupancy data.

\subsubsection{Trial Summary}

As seen from the monthly and weekly plots it is clear that the patient has generally stayed close to their target for duration in bed. Also when the patient had less than target bed occupancy, number of entry/exit in bed is greater than the target. Such information then can be used by healthcare providers to investigate what might have triggered change in sleep pattern. This will allow them to detect underline medical condition and react proactively rather than when it becomes an emergency. Such a proactive response is 
crucial to improve patient's quality of life and reduce medical cost which are the main objectives of any RPM system.

In practice GUI will be implemented on a web server in the central node where it is accessible to healthcare providers via internet with password access.

\subsection{Proposed Centralized Data Processing and Storage Architecture for Bed Occupancy RPM System}

The implemented bed occupancy RPM system shown in Figure 7.1 used distributed data processing architecture. The data from bed sensor mat is processed on a PC located in the patient's home. This requires a processing engine to be installed along with the pressure mat. Use of a single mat is sufficient for bed occupancy monitoring. It is possible to use multiple pressure mats (up to six mats providing 144 pressure sensor and able to cover entire bed) as shown in Figure 7.2(B) to monitor other physiological parameters, e.g. duration of a sit-to-stand (SiSt) transfer which is a representative measure of a person's physical mobility, bed posture, respiratory rate, movement in bed and restlessness [63], [128].

Signal processing requirements for evaluating additional parameters mentioned above are significant. If pressure mats are used in conjunction with other sensors like ECG monitor, IR camera, accelerometer etc the computing power required in the local node will increase considerably to do the signal processing locally. While it is possible to provide processing capacity by employing a high performance PC as a local node or have dedicated signal processing chip for different sensors, this will increase the cost of RPM system significantly. 
The raw data storage requirements are also very high. The data storage requirement using single mat are $\sim 120 \mathrm{MB}$ per day, for six mats it shoots up to $\sim 450 \mathrm{MB}$ per day. So backup is expensive.

As discussed in Chapter 4 the proposed centralized architecture for the RPM system is optimal allowing for it to take advantage of evolving Internet computing especially the Cloud Computing, reducing the computing, maintenance and upgrade costs.

The bed occupancy RPM system architecture used in Figure 7.1 was revised to adopt the proposed centralized data processing architecture. Employing centralized data processing architecture for bed occupancy RPM system involves raw data from pressure mat sensors to be directly sent to the central node without any processing in the local node. Feasibility of implementing centralized data processing architecture using Blackberry for transmitting data from bed mat sensors was explored. The implementation of receive/transmit app for Blackberry was successfully done by Jacques Chauvin at Carleton University. Figure 7.10 shows the adoption of bed occupancy RPM system using centralized data processing architecture.

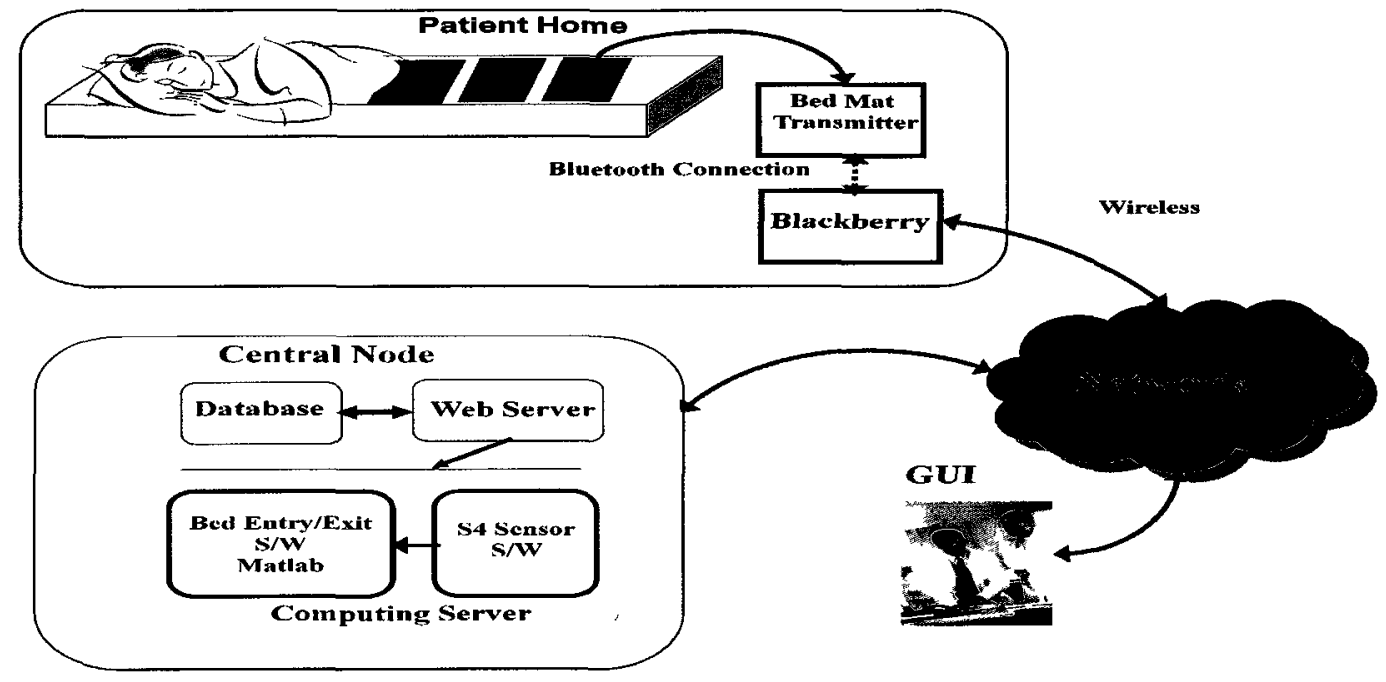

Figure 7.10: Bed Occupancy RPM System utilizing Centralized Data Processing Architecture. 
The raw mat sensor data sent by Blackberry is received at the central node and is processed by S4 Sensors software to derive the bed entry/exit data similar to what was done in the local node. This simplifies the setup needed at the patient's home significantly. All that is needed at the patient's home are the sensors and a wireless device that will receive and transmit the sensor data to the central node over wireless network. This provides ease of installation at the patient's home. Any changes, patches to processing software can be easily applied at the central node with no disruption to the patient's life. Also the raw mat data is stored at the central node for backup which is then available for future analysis; data can also be used for trialing newer algorithms.

The architecture presented in Figure 7.10 is scalable since new sensors can be added with ease.

\subsection{Case Study: Application of the AICM to Bed Occupancy RPM System}

To illustrate application of the proposed AICM method to a real life application a case study was done to show how the AICM method proposed in Chapter 5 can be applied to a bed occupancy RPM system. The case study used the bed mat sensors data to monitor the characteristics of bed-exit analysis, movement, and breathing rate.

As mentioned in Section 5.3 for centralized processing architecture signal processing of the local node must be kept to a minimum. This case study shows how very simple information content reduction methods without the need for extensive local node processing can be used improve the SI of the RPM system.

Information content reduction feasibility depends on features being extracted from the 
sensor data being monitored. It is critical to evaluate how loss of information content affects the features extracted from sensor data.

The case study demonstrates how information content can be reduced to achieve higher survivability interval under adverse network conditions and the impact of reduced information content on system performance in terms of

1. Loss of features compared with full information content.

2. Effect on specificity and sensitivity for extracted features.

The sensors used in this case study were six BOS $^{\mathrm{TM}}$ pressure sensor mats by $\mathrm{S} 4$ Sensors Inc. They provide a total of 144 sensors ( 24 sensors per mat) positioned in 8 columns across the bed and 18 rows from headboard to footboard.

Figure 7.11 shows the flow of data for the case study. Study focused on how the reduction in information content affects performance and SI for bed mat sensor data. The network transmission of the data was not part of the evaluation.

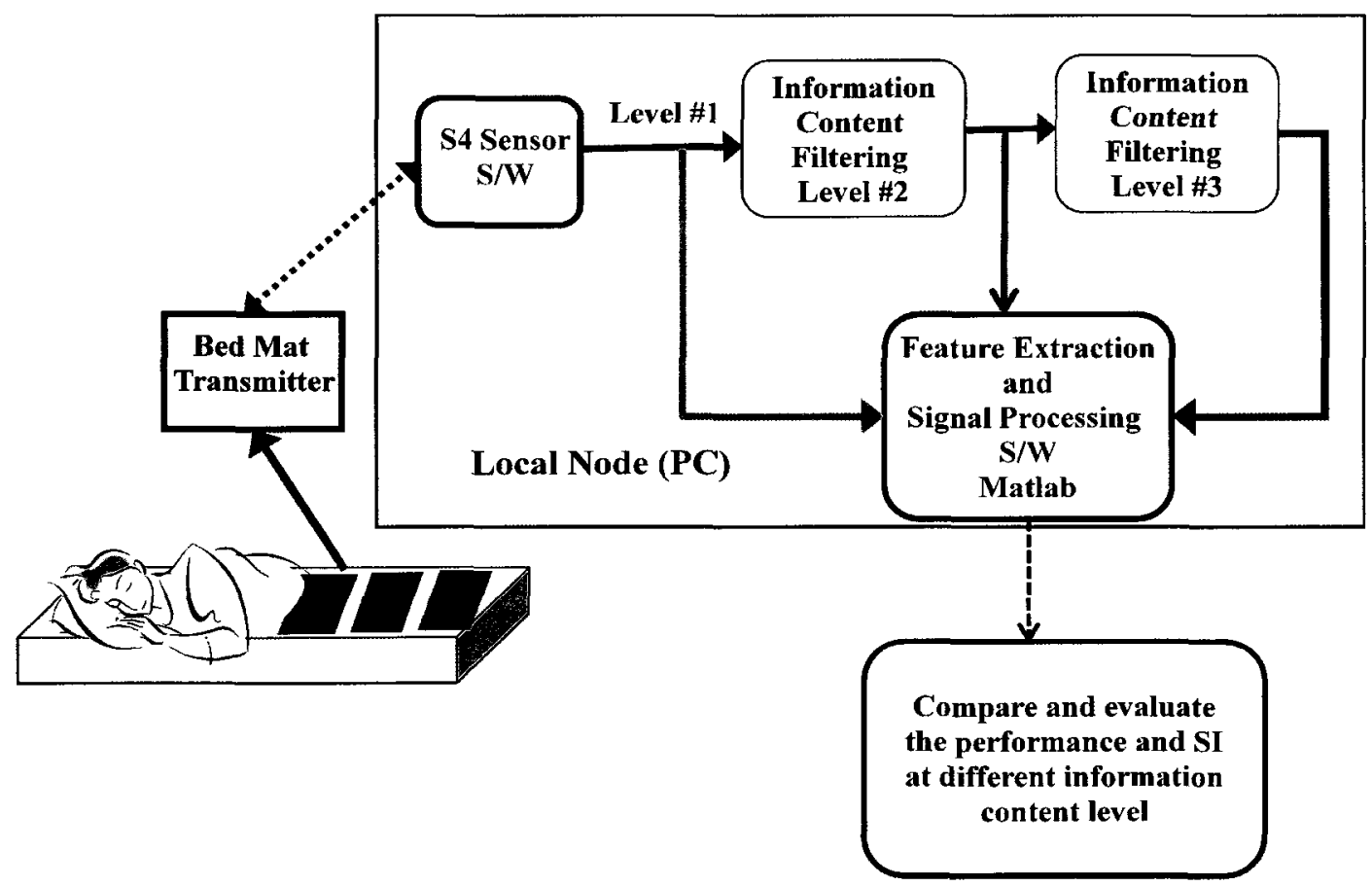

Figure 7.11: Application of AICM method to Bed Occupancy RPM System. 


\subsubsection{Clinical Feature Extraction}

Data used for the case study was collected during the trials that were conducted in the rehabilitation ward of the Élisabeth Bruyère Hospital in Ottawa, ON, Canada. Patients were monitored continuously during rehabilitation after hip surgery, beginning with the day of the operation and ending when they were released from the hospital.

The study at Élisabeth Bruyère Hospital was conducted by Amaya Arcelus and Megan Holtzman from Carleton University as a part of their research in to the patient mobility, breathing rate and balance using pressure mat. The processing algorithms for the features discussed below were also implemented by Amaya Arcelus and Megan Holtzman at Carleton University [55], [128], [129], [130], [131].

Figure 7.12 shows the clinically significant features which were extracted from the pressure-sensitive bed.

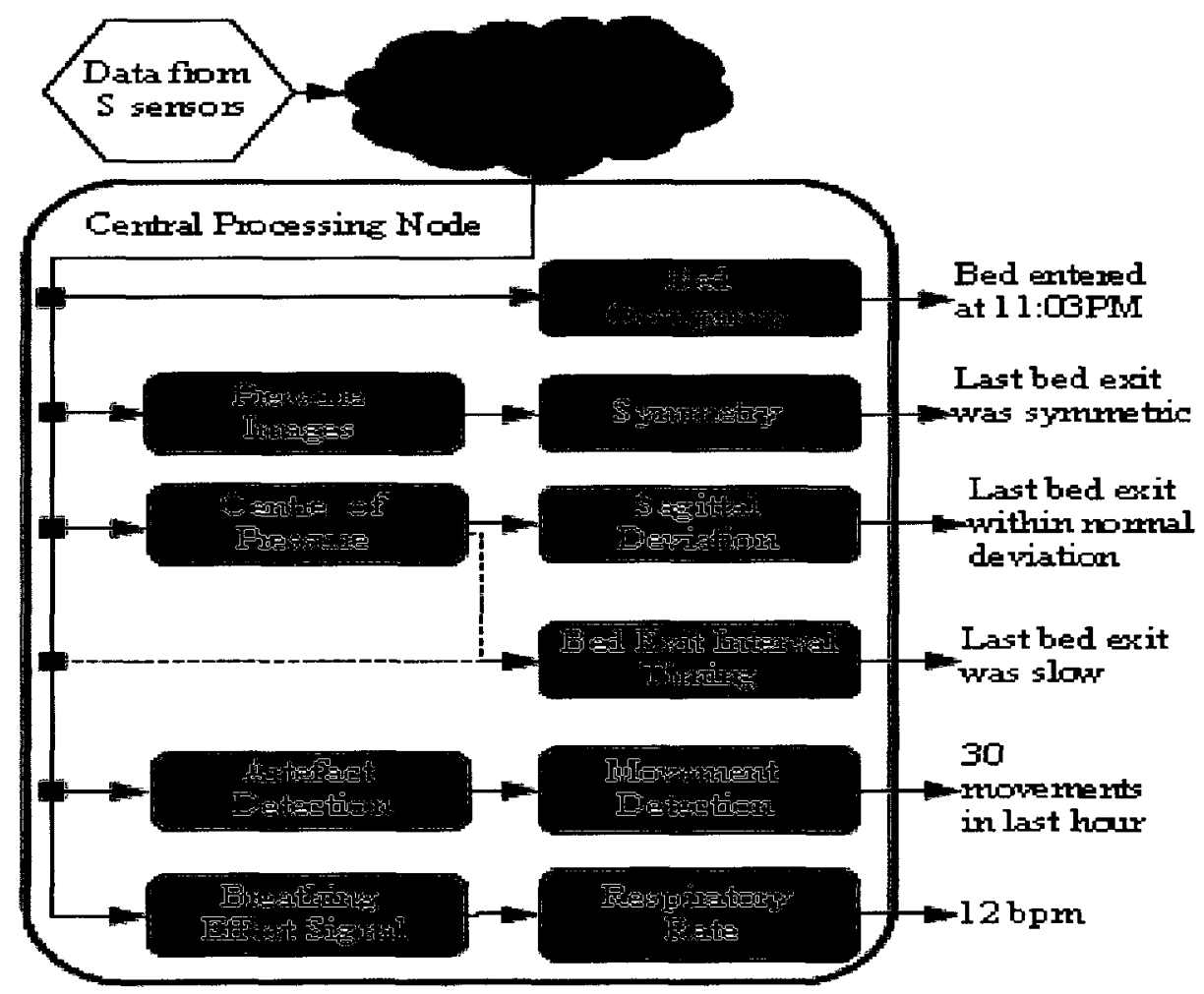

Figure 7.12: Information extraction 
Following is a brief description of each feature and the processing steps that take place to extract them.

1. Bed Occupancy

The presence of the occupant in the bed was determined using the point-source modality of the sum of all sensors [55].

2. Symmetry

The body symmetry as the occupant exited the bed was assessed by converting the sensor outputs into pressure images and then analyzing the regions of interest over time [129]. Symmetry is a significant parameter in the estimation of an occupant's level of functional mobility [132].

3. Sagittal Deviation

The deviation of the body along a sagittal plane was measured by analyzing the center of pressure trajectory as the body exits the bed. Monitoring this feature over time can indicate changes in balance and stability of an occupant [55].

4. Bed-exit Interval Timing

Timing of the interval defined by an intention to exit the bed can be measured using two methods. The first method uses the center of pressure trajectory to determine the start of the bed-exit [55]. The second method uses the sum of all sensors [130]. Timing has been shown to be a significant feature in the monitoring of home occupants [128].

\section{Movement Detection}

The detection of muscular movements was performed using the moving average and moving variance of the individual pressure signals [131]. Movement detection can be used in the measurement of sleep restlessness and overall sleep 
quality.

6. Respiratory Rate

The respiratory rate was calculated for each sensor by fusing the respiratory signals [55], and finding the highest peak in the Fourier spectrum within the frequency range of interest. Respiratory rate during sleep is widely monitored as it is a predictor of mortality in older adults [133].

\subsubsection{Content Reduction Method}

The bed occupancy RPM system shown in Figure 7.11 using six pressure mats was used to monitor and extract the six features mentioned in the previous section.

The performance and SI of the RPM system in Figure 7.11 was evaluated using the algorithms developed by Amaya Arcelus and Megan Holtzman. The proposed AICM architecture shown in Figure 7.11 uses three information content levels. When no congestion is present, data is forwarded to the local node for processing without any required buffering (Section 6.1). The following section describes how reduced information content at each level is achieved.

\subsubsection{Information Content Level 1}

Full information content is available at Level 1. At the processing node, all information is extracted once data is received. No change should be detected in the output since full information content is transmitted.

\subsubsection{Information Content Level 2}

Under moderate congestion or medium-term connectivity loss, a simple summation scheme to reduce the amount of data (and hence information) can be 
managed on the local device. To simultaneously keep centre of pressure information yet reduce the data, the sum of the rows and sum of the columns is buffered instead of the raw sensor data. This results in only $8+18=26$ data streams rather than the original 144 . The data rate reduction factor $\mathrm{D}_{2}$ is

$$
\mathrm{D}_{2}=144 / 26=5.54
$$

\subsubsection{Information Content Level 3}

When heavy congestion limits sending bandwidth, or a connection loss lasts longer than the medium buffer storage allows, only the sum of all the sensors is buffered. Since some sensors constructively interfere with each other, there would be a loss in signal to noise ratio of some extracted signals, such as the respiratory signal. Features dependent on localization would be unavailable. The data reduction factor $\mathrm{D}_{3}$ is

$$
D_{3}=144
$$

\subsubsection{Results}

\subsubsection{System Survivability}

To keep just over one second of full data content (at $10 \mathrm{KBps}$ ), a $10 \mathrm{~KB}$ buffer is allocated to the bed mat data. This is a small amount of memory, but reflects only a single component of the full RPM system buffer, total buffer available for RPM will be divided, based on needs needs of each sensor, among all the sensors. The size of buffer (10 KBs) used in this study is for illustration purpose only size of the buffer used in real RPM system is a design parameter of the system and will depend on the type of sensor, criticality of the sensor data and available network capacity. By using the proposed 
AICM method, the survivability of the system can be increased from one second with full information content to almost two and a half minutes with reduced information content. Fig. 7.13 display the time to live of the system for each information content reduction scheme [4].

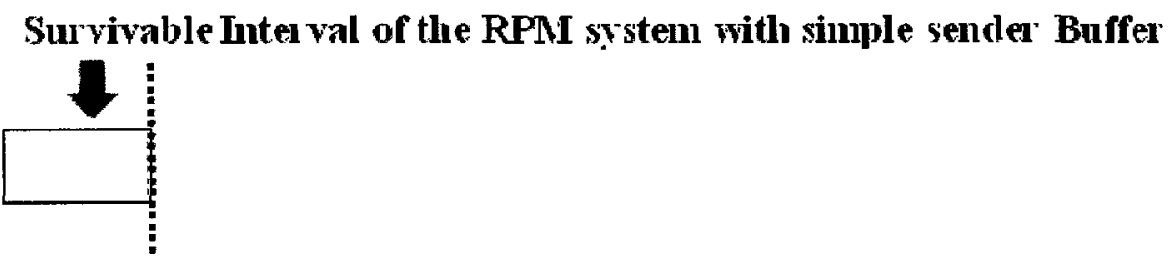

Surviwable Interval of the RPM system with AICM method

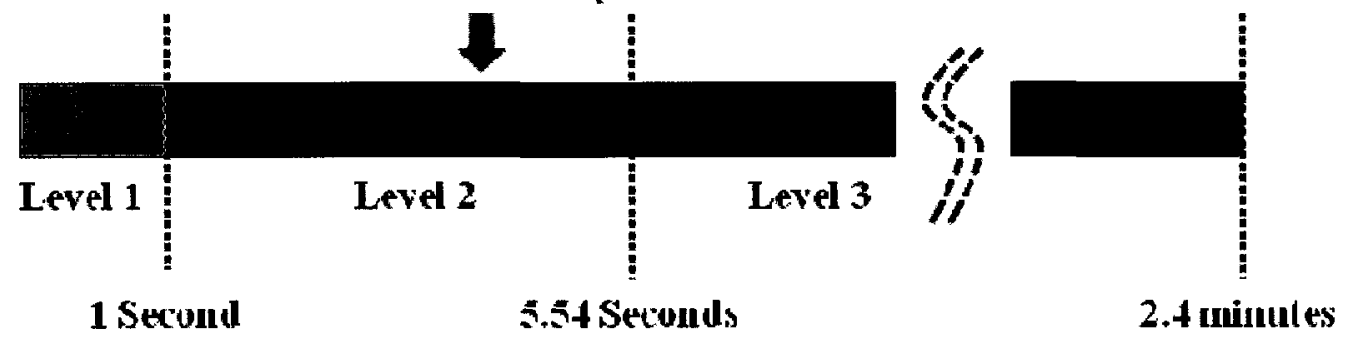

Figure 7.13: Comparison of survivability interval for bed occupancy RPM system using conventional buffering vs. one using AICM system.

Table 7.1 displays the features that are retained at successive data (and corresponding information) reduction levels using the three data reduction methods outlined in Section 7.3.2 for each level. Of the six features available with full data, two thirds of them are still available at Level 3 . However, some loss of accuracy in the features is denoted by the * marks. A total system survivability of over two and a half minutes is achieved.

The accuracy and sensitivity of the estimation and detection algorithms are dependent on the quality of the extracted movement and breathing effort signals, which are in turn dependent on the information content of the received sensor data. To compare the changes between levels, all available bed exits (31) were compared, while respiratory rates and movement detection sensitivity was compared over 12030 -second epochs. 
TABLE 7.1: Information Retained, Rate Reduction, and Survivability at each Level * indicates some loss of accuracy compared to Level 1

\begin{tabular}{|l|c|c|c|}
\hline \multicolumn{1}{|c|}{ Information Availability } & & & \\
\hline Bed Occupancy & Yes & Yes & Yes \\
\hline Bed-Exit Interval Timing & Yes & Yes & Yes* \\
\hline Sagittal Deviation & Yes & Yes & No \\
\hline Symmetry & Yes & No & No \\
\hline Movement Detection & Yes & Yes* & Yes* \\
\hline Respiratory Rate & Yes & Yes* & Yes* \\
\hline Data Rate Reduction Di & $1: 1$ & $144: 26$ & $144: 1$ \\
\hline Survivability Interval with & $1 \mathrm{sec}$ & $5.54 \mathrm{sec}$ & $2.4 \mathrm{~min}$ \\
\hline 10 KB buffer space & & & \\
\hline
\end{tabular}

Respiratory rates were counted as valid if less than five seconds of the epoch was detected as movement. Table 7.2 presents the standard deviation (s.d.) from the original feature extracted from Level 1 data at both Level 2 and Level 3. Features that are not available at those levels are marked N/A. 
Table 7.2: Standard Deviation of Features at Level 2 and 3 compared to Level 1

\begin{tabular}{|l|l|l|l|l|}
\hline Feature & $\begin{array}{l}\text { Level 2 } \\
\text { mean } \\
\text { (s.d.) }\end{array}$ & Level 2 (\%) & $\begin{array}{l}\text { Level 3 } \\
\text { mean } \\
\text { (s.d.) }\end{array}$ & \multicolumn{1}{|c|}{ Level } \\
\hline $\begin{array}{l}\text { Bed-Exit Interval } \\
\text { Timing (s) }\end{array}$ & 0 & 0 & $\begin{array}{c}12.9 \pm \\
(14.7)\end{array}$ & 29.2 \\
\hline $\begin{array}{l}\text { Sagittal Deviation } \\
\text { (cm) }\end{array}$ & 0 & 0 & N/A & N/A \\
\hline Symmetry & N/A & N/A & N/A & N/A \\
\hline $\begin{array}{l}\text { Movement } \\
\text { Detection }\end{array}$ & Sensitivity: 65.1\% & Sensitivity: & $49.4 \%$ \\
\hline $\begin{array}{l}\text { Respiratory Rate } \\
\text { (bpm) }\end{array}$ & $\begin{array}{l}0.24 \\
(0.86)\end{array}$ & $1.55(6.67)$ & $\begin{array}{l}\text { Specificity: } \\
(5.93)\end{array}$ & Specificity: 91.4\% \\
\hline
\end{tabular}

For some features, the loss of accuracy at Level 3 may be enough to warrant dropping the feature, but this depends on the application. Although movements are detected at Level 3 with a sensitivity of only $50 \%$, it is mostly small movements that are no longer discerned. The feature would still be useful for further analysis where large movements are most important, such as for position change detection.

Figure 7.14 displays the calculated bed exit interval times at all levels compared with the bed exit interval times calculated for full data at Level 1. If no modification to the results occurs due to lower information content, all of the points would lie along a $45^{\circ}$ line. Level 1 and 2 show identical results, while Level 3 is generally similar, but can be quite different, particularly for longer bed exits. 


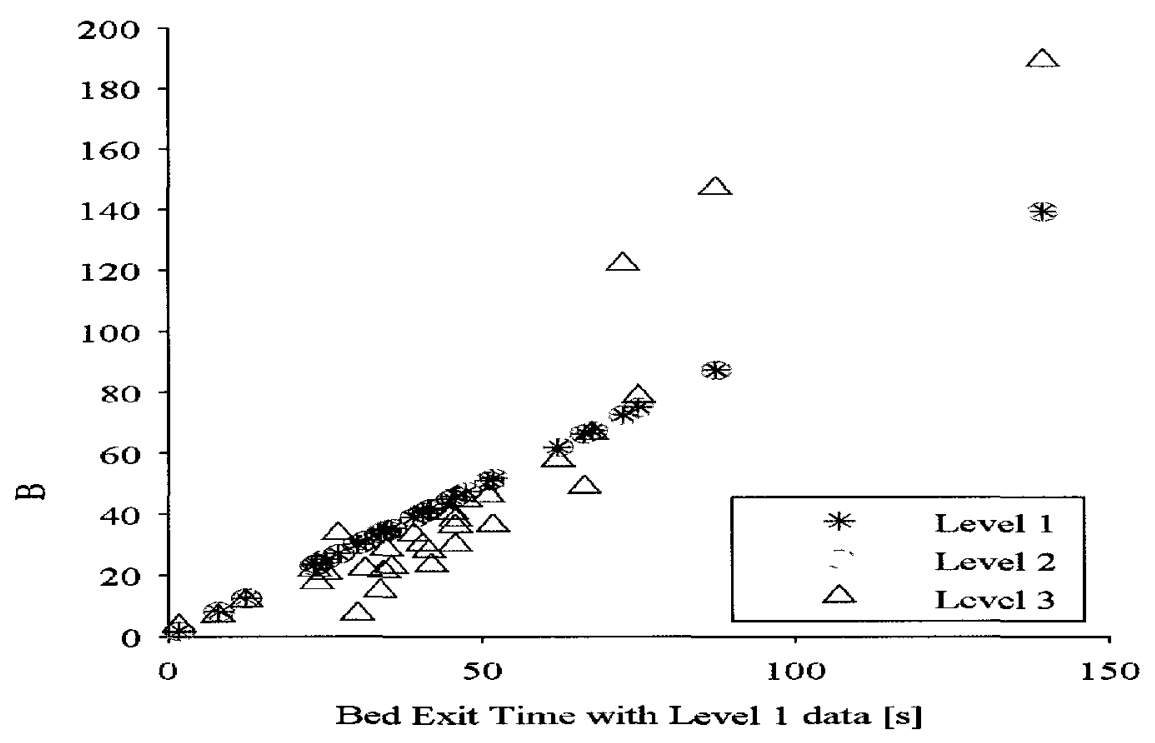

Figure 7.14: Bed exit times at all levels.

Figure 7.15 shows a similar plot for extracted respiratory rates. For Level 2 data, respiratory rates are very well correlated to the original respiratory rates at Level 1 . However, Level 3 data is less likely to properly detect movements, and some epochs at Level 3 are marked as valid, when they are in fact corrupted.

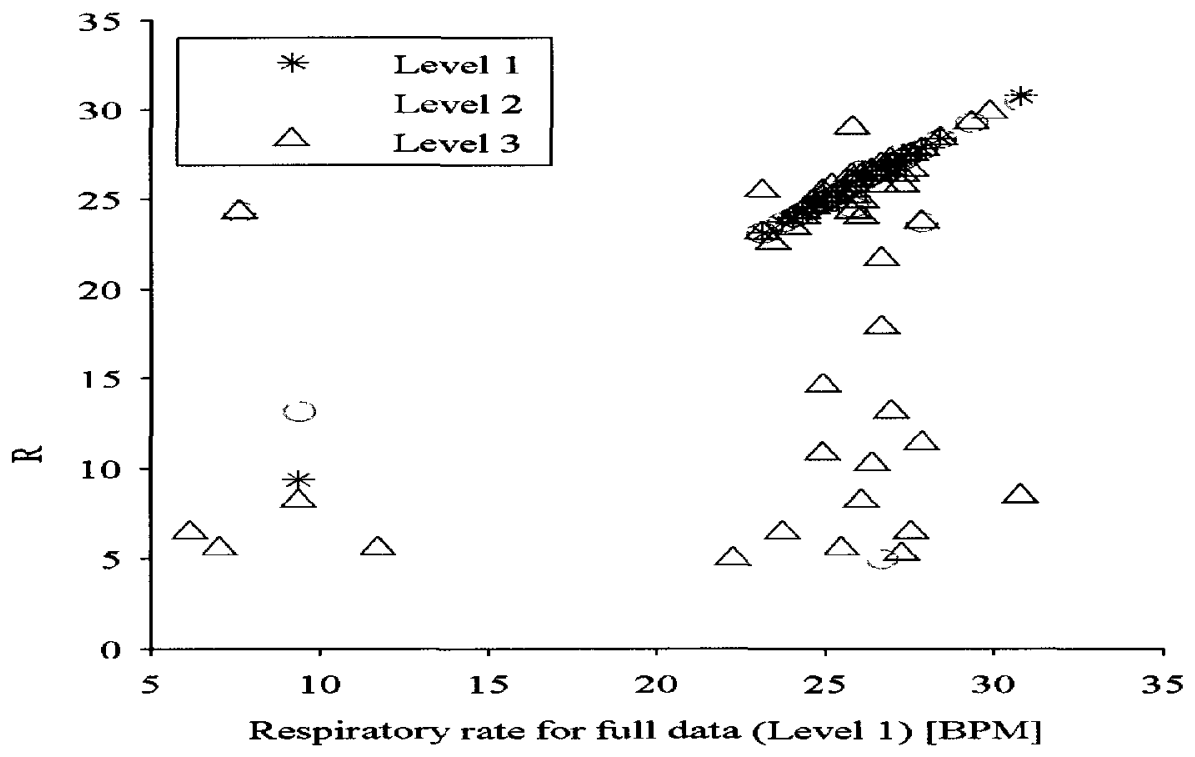

Figure 7.15: Respiratory rates at all three levels 


\subsubsection{Case Study Discussion}

The case study presented an example of how the AICM method can be used to improve the survivability of the RPM system under adverse network condition. The case study used three levels of information content to achieve data rate reduction. How many levels to use for data rate reduction depends of criticality of the data and how the loss of information content affects the features extracted for that particular sensors. As seen from case study reduction in information content, e.g. sending only the average sum during a given time period rather than all bed mat sensors data, can cause loss of certain features as well as reduce accuracy for remaining features. Each of the three levels were designed to maximize the number of surviving features; however, in reality the tradeoff between data rate reduction and loss of feature availability would be dictated by the criticality of the data to meet the patient's healthcare requirements.

For this case study the same signal processing algorithms for extracting features were used at each information content level. The algorithms did not take in to account reduction in information content. Optimizing the signal processing algorithms based on reduced information content at each level could result in better accuracy for the extracted features. For instance, to assess valid epochs at Level 3, a secondary method to assess validity, such as by analysis of the signal to noise ratio or by application of the reliability method presented in [63], could be implemented. It may be possible that a completely different set of signal processing algorithms could be used at each level to extract the same features.

The application of the AICM method to bed occupancy RPM system clearly shows that using reduced information content survivability of the system can be significantly 
improved while still retaining many of the extracted features either with same or reduced accuracy .

Similar approach can be applied to various other sensor configurations, e.g. IR video, microphone, EEG, UWB etc especially for those sensors requiring high bandwidth as a part of future work. Application of the AICM method for sensors mentioned above and design and evaluation of different algorithms for feature extractions when information content is reduced is part of the future work. 


\section{Chapter 8: Discussion}

\subsection{Adaptive Information Content Reduction}

As described in Chapter 4 centralized processing architecture provides most cost effective, mobile, transparent, non-intrusive and scalable model for the RPM system. The centralized processing architecture is feasible due to availability of broadband wireless networks like $4 \mathrm{G}$ and beyond. One of big advantage of the proposed architecture is that it is capable of seamlessly integrating with newer computing paradigm like cloud computing.

Any RPM system architecture must be reliable and robust. Robust architecture will ensure that it will survive non-catastrophic failures and the patient's health is not jeopardized.

The proposed AICM method significantly improves the survivability of the RPM system when network failures occur. This provides a robust architectural platform for RPM system.

The design considerations for the AICM method include the number of levels used, the size of sender buffer available and processing capability of the local node.

The AICM techniques must employ low fidelity signal processing methodology to achieve information content reduction. This will ensure that the local node does not need 
special processing hardware and allow common wireless devices like PDAs, cell phones etc. to act as a local node.

\subsubsection{Techniques for Information Content Reduction}

The information content of the RPM system can be reduced using different techniques based on the sensors and biological/environmental condition being monitored. Some of the factors that will affect information content reduction are the specific patient condition(s) being monitored, real-time response requirements, physical location of the patient home, e.g. rural or city; the need to reduce false alarms and the response time of the healthcare providers.

Information content reduction methods can be employed even under normal traffic conditions to reduce the bandwidth requirements of the RPM system and need not be limited to use when available network bandwidth drops due to congestion.

Use of arbitrary information rate reduction techniques like randomly dropping packets when buffer is full will result in loss of critical patient information and are not suitable for the RPM application. There has been very little research in the area of data rate reduction techniques when network performance degrades for the RPM application. This is mainly due to architectures most commonly proposed in literature for the RPM system use distributed processing architecture where raw sensor data is processed at the local node and only the results which require low bit rate are sent to the central node [134]. Also majority of the research is focused on signal processing aspects for different sensors and not overall integrated working of multitude of sensors for a comprehensive RPM system. Mohomed et al have proposed a user context aware filtering to reduce the data rate for the RPM system [135]. 
While current research in to data rate reduction for the RPM system is almost nonexistent many data rate reduction techniques have been proposed for variety of other applications which may be applicable for use with the RPM system, e.g. Streaming video/audio [136], [137], [138], Web [139], [140], Body Sensor Network (BSN) [141], [142] etc. Network congestion and loss of connectivity is faced by most of the streaming applications when transmitting over network with variable bandwidth while BSN uses data rate reduction techniques to conserve battery power. Some of the proposed content reductions techniques for Video/Audio, Web, BSN applications are mentioned below.

- Lossy compression

Lossy compression provides much higher compression ratio that lossless compression. Some analysis would have to be done to find acceptable limit of the distortion introduced by lossy compression for use in RPM system [141], [142].

- Scalable Codecs

Scalable codecs can adapt to varying network capacity. The Scalable Video Codec (SVC) is an extension of the H.264 standard (H.264 SVC) for video streaming application. Scalable codecs store information content in multiple layers. Lowest layer contains the least information content and successive layers contain increasing information content to improve the quality of the image. Which layer is transmitted depends on network capacity or limitations of user device [138].

- Downsampling

Reduction in data rate can be achieved by downsampling the sensor data by a certain factor [143]. Downsampling requires pre anti-aliasing filter. Downsampling 
decreases the data rate but will affect quality of the information. It may be possible to dynamically adjust the data transmission rate, higher data sampling rate may be triggered on specific event and low data sampling rate used at rest of the times, e.g. fall detection, coughing/snoring event etc.

- Turning off non-critical sensor data transmission

For this method, data rate reduction is limited by the data rate of the sensors which are being turned off. If the deactivated sensor's data stream is a low bit rate stream, then the reduction achieved will be marginal.

\section{- Data prioritization}

The data prioritization schemes are aimed at separating important part of the data from less important part so data can sent based on its importance [144]. 


\section{Chapter 9: Conclusions}

\subsection{Summary of Results and Contributions}

This research focused on developing a architectural framework for the RPM system that is cost effective, mobile, transparent, non-intrusive and scalable as well as capable of seamlessly transitioning to newer high bandwidth wireless networks and computing paradigms.

Following is the list of contributions made by this research:

- Proposed a centralized data processing and storage architecture for the RPM system and demonstrated that it meets all the design criteria of the RPM system.

- Proposed an Adaptive Information Content Management methodology that will significantly improve the survivability of the RPM system under adverse network conditions.

- Evaluated Survivability Interval for different reduced information content levels to demonstrate how AICM methodology can improve the survivability of the RPM system.

- Performed analysis to show the relationship between reduction in information content and number of levels used affect Survivability Interval.

- Proposed a Contiguous Buffering scheme for Adaptive Information Content Management methodology. 
- Evaluated the Survivability Interval of AICM method using Contiguous Buffering scheme. Compared the performance of Contiguous Buffering scheme with Static Buffering scheme and proved that Contiguous Buffering scheme outperformance Static Buffering scheme significantly.

- Demonstrated how to design number of levels and information reduction factor to achieve certain level of Survivability Interval under given network traffic load.

- Implemented a Bed Occupancy RPM system using pressure sensitive mat.

- Conducted usability test and qualitative analysis of the RPM system GUI prototype. The study was conducted for user groups that the system was targeted for, home care nurses, occupational therapists, and case managers. Audio/Video recordings were used to capture user feedback. User feedback will be integrated in the design and development process of the GUI in order to improve GUI design to assure a higher acceptance rate for the RPM system into the home care practices.

- Using the results of the usability study a Bed Occupancy RPM system GUI was implemented using matlab GUI package.

- A trial of the Bed Occupancy RPM system was conducted in cooperation with Wilfrid Laurier University using one volunteer participant for one week. The results were presented in [5], [6], [7], [8] and [9].

- The data from the trial was used with the designed GUI to show how the Bed Occupancy data can be used by healthcare providers to monitor the patient, understand the patient's progress against the set targets and adjust the treatment if needed. 
- A case study was done to demonstrate practical application of AICM method to the RPM system with pressure sensitive mat sensor. The case study focused on using the bed sensors to monitor the characteristics of bed-exit analysis, movement, and breathing rate. The case study demonstrates how information content can be reduced to achieve higher survivability interval under adverse network condition and the impact of reduced information content on system performance. The results have been submitted for review in [4].

\subsection{Recommendations for Future Work}

During the course of the research for this thesis it became clear that certain areas need to be investigated further to ensure that the RPM system achieves the potential benefits it offers and gain wide user acceptance. The recommendations for future work in to those areas are summarized here:

- Further work needs to be done to investigate information content reduction techniques for various sensors especially ones' requiring high bandwidth, e.g. IR, video, microphone; similar to one done for pressure sensitive mat in this thesis. This is critical if the RPM system is to meet its high availability requirement since not all the sensors or all the sensor data from a particular sensor is real-time critical and how the RPM system handles adverse network condition without jeopardizing patient's health will have significant ramification on its wider usage.

- Many signal processing algorithms have been proposed to monitor variety of medical conditions using different sensors. All of these signal processing algorithms assume full bandwidth data being available from the sensors. It is important to investigate the performance of these algorithms when information 
content from the sensor data is reduced. It is not clear if all the proposed signal processing algorithms would be optimal when information content in the sensor data is reduced. Different signal processing algorithms may perform better with reduced information content. It may lead to the RPM system design where different sets of algorithms of used for same sensor depending on the information content reduction factor and must be researched.

- The use of Internet for the RPM system connectivity puts major onus on network QoS issue. The QoS requirements and protocols specifically suitable for RPM systems must be investigated. 


\section{References}

[1] United Nations Department of Economic and Social Affairs Population Division. (2002). World population ageing: 1950-2050 [Online]. Available: http://www.un.org/esa/population/publications/worldageing19502050/

[2] Center for Technology and Aging. (2009, Dec.). Technologies for Remote Patient Monitoring in Older Adults [Online]. Available:

http://www.techandaging.org/RPMpositionpaperDraft.pdf

[3] R.S.H. Istepanian, C.S. Pattichis and S. Laxminarayan, "Ubiquitous M-Health Systems and the Convergence Towards 4G Mobile Technologies". in Topics in Biomedical Engineering. International Book Series, Springer-Verlag New York Inc. 2006, pp. 3-14.

[4] V. Joshi, A. Arcelus, M. Holtzman, R. Goubran and F. Knoefel, "Adaptive Information Content Management and Buffer Caching Design for Robust Remote Patient Monitoring System," IEEE Trans. on Information Technology in Biomedicine, (under review).

[5] W. Sun, H. Nguyen, D.M. Doran, R. Goubran, S. Doherty, F. Knoefel, A. Tosine, V. Joshi, and E. Sadowski, "Integration of Bio-mobility Information with at Pointof-Care Decision Support System for Safer Client Care," presented at the Canadian Nursing Informatics Association, Mississauga, ON, Nov. 22-25, 2009.

[6] W. Sun, H. Nguyen, D.M. Doran, R. Goubran, S. Doherty, F. Knoefel, A. Tosine, V. Joshi, and E. Sadowski, "Integration of Bio-mobility Information with at Pointof-Care Decision Support System for Safer Client Care," presented at the Sigma Theta Tau 23rd Annual Research Conference: 'Promoting Excellence in Nursing Practice Through Research'. London, ON, Apr. 30, 2010.

[7] H. Nguyen, D.M. Doran, R. Goubran, S. Doherty, F. Knoefel, A. Tosine, H. Nguyen, V. Joshi, W. Sun, and E. Sadowski, 'Integration of Bio-mobility 
Information with at Point-of-Care Decision Support System for Safer Client Care," presented at the 4th National Community Health Nurses Conference, Toronto, ON, June 16-18, 2010.

[8] D. Doran, R. Goubran, S. Doherty, F. Knoefel, A. Tosine, H. Nguyen, V. Joshi, W. Sun and E. Sadowski, "Technology Integration Into Home Care Nursing: Meeting the Challenges of Chronic Disease Management," poster presentation at e-Health 2010, Vancouver, BC, May 30 - June 3, 2010.

[9] A. Tosine, H. Nguyen, V. Joshi, D. Doran, A. Parush, R. Goubran, S. Doherty and E. Sadowski, "User Research of a Proposed Home Care Clinician's Nonintrusive Remote Monitoring System's Graphical User Interface," poster presentation at e-Health 2010, Vancouver, BC, May 30-June 3, 2010.

[10] World Health Organization. (2005). Preventing chronic diseases: A vital investment [Online]. Available:

http://www.who.int/chp/chronic disease_report/en/

[11] The Milken Institute. (2007, Oct.). An Unhealthy America: The Economic Burden of Chronic Disease Charting a New Course to Save Lives and Increase Productivity and Economic Growth [Online]. Available: http://www.milkeninstitute.org/healthreform/pdf/AnUnhealthyAmericaExecSum $\underline{\text { m.pdf }}$

[12] L. O.Brien-Pallas, C. Alksnis, S. Wang. (2003). Bringing the Future into Focus: Projecting RN Retirement in Canada [Online]. Available at: http://dsp-psd.communication.gc.ca/Collection/H118-12-2003E.pdf

[13] Associations of American medical Colleges. (2010, Sep.). Physician Shortages to Worsen Without Increases in Residency Training [Online]. Available: http://www.aamc.org/advocacy/library/teachhosp/corres/2009/102709.pdf

[14] Center for Disease Control and Prevention. Chronic Diseases and Health Promotion [Online]. Available: http://www.cdc.gov/chronicdisease/index.htm.

[15] D. Simons, T. Egami, and J. Perry, "Remote Patient Monitoring Solutions," Advances in Health care Technology Care Shaping the Future of Medical Care, Springer Netherlands, 2006, Ch. 30.

[16] Gartner. (2009). eHealth for a Healthier Europe! opportunities for a better use of healthcare resources [Online]. Available:

http://www.se2009.eu/polopoly fs/1.8227!menu/standard/file/eHealth\%20for $\% 20$ a\%20Healthier\%20Europe.pdf 
[17] Ontario Government Ministry of Finance. (2010). Ontario's Long-Term Report on the Economy [Online]. Available:

http://www.fin.gov.on.ca/en/economy/ltr/2010/ch3.html

[18] Vital Wave Consulting. (2009). mHealth for Development: The Opportunity of Mobile Technology for Healthcare in the Developing World [Online]. Available: http://www.unfoundation.org/global-issues/technology/mhealth-report.html

[19] C. P. Waegemann, "mHealth: The Next Generation of Telemedicine?," Telemedicine and e-Health, vol.16, no. 1, pp. 23-25, Jan./Feb. 2010.

[20] P. N. Mechael, "The Case for mHealth in Developing Countries," MIT Press Journal Innovations: Technology, Governance, Globalization, vol. 4, no. 1, pp. 108-113, Jan. 2009.

[21] U. Varshney, "A framework for supporting emergency messages in wireless patient monitoring," Decision Support Systems, vol. 45, no. 4, pp. 981-996, Nov. 2008.

[22] S. Junnila, H. Kailanto, J. Merilahti, A. Vainio, A. Vehkaoja, M. Zakrzewski, and J. Hyttinen, "Wireless, multi-purpose in-home health monitoring platform: two case trials," IEEE Trans. Information Technology in Biomedicine. vol. 14, no. 2 , pp. 447 - 455, Mar. 2010.

[23] E Kyriacou, S Pavlopoulos, A Berler, M Neophytou, A Bourka, A Georgoulas, A Anagnostaki, D Karayiannis, C Schizas, C Pattichis, A Andreou and D Koutsouris, (2003, Mar.), "Multi-purpose HealthCare Telemedicine Systems with mobile communication link support," BioMedical Engineering OnLine, [Online], Available: $\underline{\mathrm{http}: / / \mathrm{www} . \text { biomedical-engineering-online.com/content/2/1/7 }}$

[24] C-J Su and C-Y Wu, "JADE implemented mobile multi-agent based, distributed information platform for pervasive health care monitoring," Applied Soft Computing, vol. 11, no. 1, pp. 315-325, Jan. 2011.

[25] Juniper Research. (2010, Mar.). Mobile Healthcare Opportunities Monitoring, Applications \& mHealth Strategies 2010 - 2014 [Online]. Available: http://www.juniperresearch.com/reports/mobile healthcare_opportunities

[26] C. Orwat, A. Graefe and T. Faulwasser, "Towards pervasive computing in health care - A literature review," BMC Medical Informatics and Decision Making, Volume 8, Number 1, June 2008, pp 1-18. 
[27] M. J. Field; J. Grigsby, "Telemedicine and Remote Patient Monitoring," JAMA: The Journal of the American Medical Association, vol. 288, no. 4, pp. 423-425, July 2002.

[28] P. E. Ross, "Managing care through the air [remote health monitoring]," IEEE Spectrum, vol. 41 no. 12, pp 26-31, Dec. 2004.

[29] T. Bratan and M. Clarke, "Optimum Design of Remote Patient Monitoring Systems," in Proc. $28^{\text {th }}$ Int. Conf. IEEE Eng. Med. Biol. Soc., New York, NY, USA, 2006, pp. 6465-6468.

[30] S. Sneha and U. Varshney, "Enabling ubiquitous patient monitoring: Model, decision protocols, opportunities and challenges," Decision Support Systems, vol.46, no. 3, pp. 606-619, Feb. 2009.

[31] J. Basilakis, N.H. Lovell, S.J. Redmond, and B.G. Celler, "Design of a decisionsupport architecture for management of remotely monitored patients," IEEE Trans. Information Technology in Biomedicine, vol. 14, no. 5 pp. 1216-1226, Sept. 2010.

[32] M. P. Rajasekaran, S. Radhakrishnan and P. Subbaraj, "Sensor grid applications in patient monitoring," Future Generation Computer Systems, vol. 26, no. 4, pp. 569-575, Apr. 2010.

[33] L. P. Aroul, W. Walker, D. Bhatia. (2006). A Framework for Patient Monitoring [Online]. Available:

http://www.motorola.com/innovators/ModusWorkshop/A_Framework.pdf

[34] A. Hein, O. Nee, D. Willemsen, T. Scheffold, A. Dogac, G. B. Laleci, "SAPHIRE - Intelligent Healthcare Monitoring based on Semantic Interoperability Platform The Homecare Scenario," 1st European Conference on eHealth (ECEH'06), Fribourg, Switzerland, Oct. 2006.

[35] C. N. Scanaill, S. Carew, P. Barralon, N. Noury, D. Lyons and G. M. Lyons, "A Review of Approaches to Mobility Telemonitoring of the Elderly in Their Living Environment," Annals of Biomedical Engineering, vol. 34, no. 4, pp. 547-563, Apr. 2006.

[36] C-T. Lin; K-C. Chang; C-L. Lin; C-C. Chiang; S-W. Lu; S-S. Chang; B-S. Lin; HY. Liang; R-J. Chen; Y-T. Lee; and L-W. Ko, "An Intelligent Telecardiology System Using a Wearable and Wireless ECG to Detect Atrial Fibrillation," IEEE Trans. Information Technology in Biomedicine. vol. 14, no. 3, pp. 726-733, May 2010. 
[37] DH. Shih, HS Chiang, B. Lin, and SB. Lin, "An embedded mobile ECG reasoning system for elderly patients," IEEE Trans. Information Technology in Biomedicine, vol. 14, no. 3, pp. 854- 865, May 2010.

[38] S. Kumar, K. Kambhatla, F. Hu, M. Lifson and Y. Xiao, "Ubiquitous Computing for Remote Cardiac Patient Monitoring: A Survey," International Journal of Telemedicine and Applications, vol. 2008, Jan. 2008.

[39] A. Alesanco and J. Garcia, "Clinical Assessment of Wireless ECG Transmission in Real-Time Cardiac Telemonitoring," IEEE Trans. Information Technology in Biomedicin, vol. 14, no. 5 pp. 1144-1152, Sep. 2010.

[40] H. Burri and D. Senouf, "Remote monitoring and follow-up of pacemakers and implantable cardioverter defibrillators," Europace, vol. 11, no. 6, pp. 701-709, June 2009.

[41] K. Malakuti, "Towards an Intelligent Bed Sensor: Non-intrusive Monitoring of Sleep Disturbances Via Computer Vision Techniques, M.A.Sc. thesis, Univ. of Victoria, Victoria, B.C., 2008.

[42] Chih-Ming Cheng, Yeh-Liang Hsu, and Chang-Ming Young, "Development of a Portable Device for Telemonitoring of Physical Activities During Sleep," Telemedicine and e-Health, vol. 14, no. 10, pp. 1044-1056, Dec. 2008.

[43] J. Biswas, M. Jayachandran, L. Shue, K. Gopalakrishnan and P. Yap, "Design and Trial Deployment of a Practical Sleep Activity Pattern Monitoring System," proc. of the 7th Int. conf. on Smart Homes and Health Telematics: Ambient Assistive Health and Wellness Management in the Heart of the City, Tours, France, 2009, pp. $190-200$.

[44] J. Tilmanne, J. Urbain, M. V. Kothare, A. V. Wouwer, S. V. Kothare, "Algorithms for sleep-wake identification using actigraphy: a comparative study and new results," Journal of Sleep Research, vol. 18, no. 1, pp. 85-98, Mar. 2009.

[45] X. Zhu, W. Chen, T. Nemoto, Y. Kanemitsu, K. Kitamura, K. Yamakoshi, and D. Wei, "Real-Time Monitoring of Respiration Rhythm and Pulse Rate During Sleep," IEEE Trans. Biomed. Eng., vol. 53, no. 12, pp. 2553-2563, Dec. 2006.

[46] J. Merilahti, A. Saarinen, J. Pärkkä, K. Antila, E. Mattila and I. Korhonen, "Long Term Subjective and Objective Sleep Analysis of Total Sleep Time and Sleep Quality in Real Life Settings," in Proc. $28^{\text {th }}$ Int. Conf. IEEE Eng. Med. Biol. Soc., Lyon, France, 2009, pp. 5202-5205. 
[47] J. H. Shin, Y. J. Chee, D. Jeong, and K. S. Park, "Non-constrained Sleep Monitoring System and Algorithms using Air-mattress with Balancing Tube Method," IEEE Trans. on Information Technology in Biomedicine, vol. 14, no.1, pp. 147-156, Jan. 2010.

[48] Matt Van Wieringen, J. Mikael Eklund, "Real-Time Signal Processing of Accelerometer Data for Wearable Medical Patient Monitoring Devices," in Proc. $28^{\text {th }}$ Int. Conf. IEEE Eng. Med. Biol. Soc., Vancouver, BC, 2008, pp. 2397-2400.

[49] W. Chen, X. Zhu, T. Nemoto, K. Kitamura, K. Sugitani and D. Wei, "Unconstrained monitoring of long-term heart and breath rates during sleep," Physiological Measurement, vol. 29, no. 2, Feb. 2008, pp. N1-N10.

[50] Y-T. Peng, C.-Y. Lin, and M.-T. Sun, "A Distributed Multimodality Sensor System for Home-Used Sleep Condition Inference and Monitoring," in Proc.of the 1st Transdisciplinary Conf. on Distributed Diagnosis and Home Healthcare (D2H2), Arlington, Virginia, USA, 2006, pp. 20-23.

[51] W D Duckitt, S K Tuomi and T R Niesler, "Automatic detection, segmentation and assessment of snoring from ambient acoustic data," Physiological Measurement, vol. 27, no. 10, Oct. 2006, pp. 1047-1056.

[52] C.J. James, J. Crowe, E. Magill, S.C. Brailsford, J. Amor, P. Prociow, J. Blum and S. Mohiuddin, "Personalised Ambient Monitoring (PAM) of the mentally ill," in Proc. of 4th European Conference of the International Federation for Medical and Biological Engineering, Antwerp Belgium, Nov. 2008, pp 1010-1013.

[53] M. Popescu and A. Mahnot, "Acoustic Fall Detection Using One-Class Classifiers," in Proc $31^{\text {st }}$ Int. Conf. IEEE Eng. Med. Biol. Soc, Minneapolis, Minnesota, USA, 2009, pp. 3505-3508

[54] A. Sixsmith, N. Johnson, "A smart sensor to detect the falls of the elderly," IEEE Pervasive Computing, vol. 3, no. 2, pp. 42 - 47, Apr.-June 2004

[55] A. Arcelus, R. Goubran, H. Sveistrup, M. Bilodeau and F. Knoefel, "Contextaware smart home monitoring through pressure measurement sequences," submitted to IEEE Trans. Instrum. Meas. Jul. 2010.

[56] N. Fourty, D. Guiraud, P. Fraisse, G. Perolle, I. Etxeberria and T. Val, "Embedded system used for classifying motor activities of elderly and disabled people," Computers \& Industrial Engineering, vol. 57, no.1, pp. 419-432, Aug. 2009.

[57] M. Y. Yuan, "Using Thermal Imaging to Promote Independent Living," M.A.Sc thesis, Dept. of Sys. and Comp. Eng., Carleton Univ, Ottawa, ON, 2008. 
[58] A. R. Kaushik and B.G. Celle, "Characterization of PIR detector for monitoring occupancy patterns and functional health status of elderly people living alone at home," Technology and Health Care, vol. 15, no. 4, pp. 273-288, Sept. 2007.

[59] M. Hamill, V. Young, J. Boger and A. Mihailidis, "Development of an automated speech recognition interface for personal emergency response systems," Journal of NeuroEngineering and Rehabilitation, vol. 26, no. 6, July 2009.

[60] D. Istrate, E. Castelli, M. Vacher, L. Besacier, and J. Serignat, "Information Extraction From Sound for Medical Telemonitoring," IEEE Trans. on Information Technology in Biomedicine, vol. 10, no. 2, pp. 264-274, Apr. 2006.

[61] D.Istrate, M. Binet and S. Cheng, "Real Time Sound Analysis for Medical Remote Monitoring," in Proc. $31^{\text {st }}$ Int. Conf. IEEE Eng. Med. Biol. Soc, Vancouver, BC, 2008, pp. 4640-4643.

[62] M. Okubo, Y. Imai, T. Ishikawa, T. Hayasaka, S. Ueno and T. Yamaguchi, "Development of automatic respiration monitoring for home-care patients of respiratory diseases with therapeutic aids," in Proc. 4th European Conf. of the International Federation of Medical and Biological Engineering, ECIFMBE, 2008, pp.1117-1120.

[63] M. H. Jones, R. A. Goubran, and F. Knoefel, "Reliable respiratory rate estimation from a bed pressure array," in Proc. $28^{\text {th }}$ Int. Conf. IEEE Eng. Med. Biol. Soc., New York, NY, USA, 2006, pp. 6410-6413.

[64] A. Copetti, O. Loques, J. C. B. Leite, T. P. C. Barbosa, A. C. L. da Nobrega, "Intelligent Context-Aware Monitoring of Hypertensive Patients," in Proc. Of $3^{\text {rd }}$ Int. Conf. Pervasive Computing Technologies for Healthcare, London, UK, 2009, pp. 1-4.

[65] A. K. Whitchurch, J. K. Abraham and V. K. Varadan, "Design and Development of a Wireless Remote Point-of-Care Patient Monitoring System," in Proc. of IEEE Region 5 Technical Conf., Fayetteville, Arkansas, USA, 2007, pp. 163 - 166.

[66] C. Gerigan, P. Ogrutan and L. Aciu, "Remote EEG Monitoring," in Proc. of $15^{\text {th }}$ Int. IEEE Symposium Design and Technology of Electronics Packages, (SIITME), Gyula, Hungary 2009, pp. $271-276$.

[67] Wave Technology Group. (2010). Wave EEG Monitor [Online]. Available: http://wavetechnologygroup.net/solutions/medical/health-monitoring/eeg. 
[68] Vitaphone. (2010). Vitaphone-Tele-ECG-Loop-Recorder [Online]. Available: http://www.vitaphone.de/en/products/telecardiological-diagnostics/looprecorder.html

[69] Emfit Ltd. Non Contact Vitals Monitor and IP Nurse-Call System [Online]. Available: http://www.emfit.com/en/care/products care/vitals-monitor-and-nurse$\underline{\text { call } /}$

[70] Biotronik. The New BIOTRONIK Home Monitoring [Online]. Available: http://www.biotronik.com/biohm/the-new-biotronik-home-monitoring/18593

[71] Boston Scientific Corp. The LATITUDE® Patient Management system [Online]. Available: http://www.bostonscientificinternational.com/Device.bsci?page $=$ HCP Overview\&navRelId $=1000.1003 \&$ met $\underline{\text { hod=DevDetailHCP\&id=10118992\&pageDisclaimer=Disclaimer.ReservedForMe }}$ dProfs, \%20Disclaimer.ProductPage

[72] Medtronic Inc. CareLink Home Monitor [Online]. Available: http://www.medtronic.com/for-healthcare-professionals/productstherapies/cardiac-rhythm/patient-management-carelink/medtronic-carelinknetwork-for-cardiac-device-patients/index.htm

[73] St. Jude Medical Inc. Merlin@home® Transmitter [Online]. Available: http://www.sjmprofessional.com/Products/US/CRT-Systems/Merlin-at-homeTransmitter.aspx

[74] Telemedcare Ltd. (2009). TeleMedCare Health Monitor [Online]. Available: http://www.telemedcare.com.au/index.php/equipment

[75] A. Pantelopoulos and N. G. Bourbakis, "A Survey on Wearable Sensor-Based Systems for Health Monitoring and Prognosis," IEEE Trans. on Systems, Man, and Cybernetics, Part C: Applications and Reviews, vol. 40, no. 1, pp. 1-12, Jan. 2010.

[76] D.Berian, V. Stoicu-Tivadar, V. Gomoi, "Technical Aspects of Implementing a Telecare System," in Proc. of IEEE Int. Joint Conf. on Computational Cybernetics and Technical Informatics (ICCC-CONTI 2010), Timisora, Romania, 2010, pp. 237-241.

[77] M. A. Valero, L. Vadillo, I. Pau, and A. Peñalver, "An Intelligent Agents Reasoning Platform to Support Smart Home Telecare," Distributed Computing, Artificial Intelligence, Bioinformatics, Soft Computing, and Ambient Assisted Living, vol. 5518, pp. 679-686, 2009. 
[78] J. S. Wilson, "Sensor technology handbook, Volume 1," Newnes, Burlington Massachusetts, USA, 2005.

[79] Kris Cuppens, Lieven Lagae, Berten Ceulemans, Sabine Van Huffel, and Bart Vanrumste, "Detection of nocturnal frontal lobe seizures in pediatric patients by means of accelerometers: a first study," in Proc. $31^{\text {st }}$ Int. Conf. IEEE Eng. Med. Biol. Soc, Minneapolis, Minnesota, USA, Sep. 2009, pp. 6608-6611.

[80] A. H. Nasution and S. Emmanuel, " Intelligent Video Surveillance for Monitoring Elderly in Home Environments," in $9^{\text {th }}$ Workshop IEEE Multimedia Signal Processing MMSP, Crete, Greece, Oct. 2007, pp. 203-206.

[81] H. C. Keong and M. R. Yuce, "Evaluation of UWB receiver position for Wireless Body Area Network," in the IEEE Int. conf. on Ultra-wideband (ICUWB2010), Sept. 2010.

[82] Z. Jinyun, P.V. Orlik, Z. Sahinoglu, A. F. Molisch, P. Kinney, "UWB Systems for Wireless Sensor Networks," in Proc. of IEEE, vol. 97, no. 2, pp. 313-331, February 2009.

[83] Continua Health Alliance. [Online]. Available: http://www.continuaalliance.org/index.html

[84] D. D. Vergados, "Simulation and Modeling Bandwidth Control in Wireless Healthcare Information Systems," Simulation, vol. 83, no. 4, pp. 347-364, Apr. 2007

[85] Cisco Systems. Quality of Service Networking [Online]. Available: http://www.cisco.com/en/US/docs/internetworking/technology/handbook/QoS.ht $\underline{\mathrm{ml} \# \mathrm{wp} 1020632}$

[86] Cisco Systems. Cisco IP Solution Center, 3.0: Quality of Service Management User Guide, 3.0 [Online]. Available:

http://www.cisco.com/en/US/docs/net_mgmt/ip_solution_center/3.0/qos/user/guid e/QoSguide.pdf

[87] Nortel Networks, "Essentials of Real-Time Networking." 2004.

[88] Nortel Networks. (2003). Introduction to Quality of Service (QoS) [Online]. Available:

http://www.nortel.com/products/02/bstk/switches/bps/collateral/56058.25 022403 .pdf 
[89] P. Nanda and R. C. Fernandes, "Quality of Service in Telemedicine," in Proc. Of 1st Int. Conf. IEEE Digital Society (ICDS'07), Guadeloupe, French Caribbean, 2007, pp 2-4.

[90] A. Zvikhachevskaya, G. Markarian and L. Mihaylova, "Quality of Service consideration for the wireless telemedicine and e-health services, " in Proc. of Wireless Communications \& Networking Conference, IEEE Comm. Soc, Budapest, Hungary, 2009.

[91] F. Hu, Y. Xiao, Q. Hao, "Congestion-Aware, Loss-Resilient Bio-Monitoring Sensor Networking for Mobile Health Applications," IEEE Journal on Selected Areas in Communications, vol. 27, no. 4, pp. 450-465, May 2009.

[92] B. Zhou, C. Hu, H.B. Wang, R. Guo, M.Q.H. Meng, "A wireless sensor network for pervasive medical supervision," in Proc. of Int. Conf. IEEE Integration Technology, Shenzhen, China, Mar. 2007, pp. 740 - 744.

[93] P. Neves, M. Stachyra, and J. Rodrigues, "Application of Wireless Sensor Networks to Healthcare Promotion," JCOMSS - Journal of Communications Software and Systems, vol. 4, no. 3, pp. 181-190, Sept. 2008.

[94] J-S Lee, Y-W Su, and C-C Shen, "A Comparative Study of Wireless Protocols: Bluetooth, UWB, ZigBee, and Wi-Fi," The 33rd Annual Conference of the IEEE Industrial Electronics Society (IECON), Nov. 2007.

[95] A. Kailas, M. A. Ingram, "Wireless Aspects of Telehealth," Wireless Personal Communication, vol. 51, no. 4, pp: 673-686, Dec. 2009.

[96] S. Dean. (2009). Taking advantage of opportunities and challenges in medical electronics [Online]. Available: http://www.embedded-systems.com/design/219100162?printable=true

[97] Texas Instruments. Wireless Connectivity for Medical Wireless [Online]. Available: http://www.arrownac.com/events-training/training/pdfs/wireless.pdf

[98] Bluetooth SIG Inc. [Online]. Available: http://www.bluetooth.com/English/Pages/default.aspx

[99] ZigBee Alliance. [Online]. Available: http://www.zigbee.org/

[100] IEEE 802 LAN/MAN Standards Committee. (2011, May). IEEE 802.15 WPAN Low Rate Alternative PHY Task Group 4a (TG4a) [Online]. Available: http://www.ieee802.org/15/pub/TG4a.html 
[101] J. Zhang, Z. Sahinoglu, P. Kinney, "UWB Systems for Wireless Sensor Networks," Proc. IEEE, vol. 97, no. 2, pp. 313-331, Feb. 2009.

[102] Z. Ahmadian, L. Lampe, "Performance Analysis of the IEEE 802.15.4a UWB System," IEEE Trans. Commun., vol. 57, no. 5, pp. 1474-1485, May 2009.

[103] H. C. Keong, M. R. Yuce, T. S. P. See and T. M. Chiam, "On-Body Evaluation of UWB Receiver position for Wireless Body Area Network," in Proc. IEEE Int. Conf .on Ultra-wideband (ICUWB2010), Nanjing, China, Sep. 2010.

[104] A. Chehri and H. Mouftah," Performance Analysis of UWB Body Sensor Networks for Medical Applications," Second Int. Conf. on Ad Hoc Networks (ADHOCNETS 2010), Victoria, BC, Aug. 2010, pp 471-481.

[105] IEEE 802 LAN/MAN Standards Committee. (2011, May). IEEE 802.15 WPAN Task Group 6 (TG6) Body Area Networks [Online]. Available: http://www.ieee802.org/15/pub/TG6.html

[106] T. Bradley. (2010, Mar. 24). Sprint 4G Broadband: A Business Primer [Online]. Available: http://www.pcworld.com/businesscenter/article/192244/sprint 4g broadband a b usiness primer.html

[107] B. Levine. (2010, Sep). AT\&T, Verizon Prepare To Compete in $4 G$ Service [Online]. Available:

http://www.sci-tech-today.com/news/AT-T--Verizon-Prepare-for-4GService/story.xhtml?story_id=10100CCFUF2S

[108] S. Ezell, R. Atkinson, D. Castro and G Ou. \{2009, Mar.). The Need for Speed: The Importance of Next-Generation Broadband Networks [Online]. Available: http://www.itif.org/files/2009-needforspeed.pdf

[109] H. Falaki, D. Lymberopoulos, R. Mahajan, S. Kandula and D. Estrin, "A First Look at Traffic on Smartphones," Internet Measurement Conference, Melbourne, Australia, Nov. 2010.

[110] Cisco Systems. (2009, Jan.). Visual Networking Index: Global Mobile Data Traffic Forecast Update, 2009-2014 [Online]. Available: http://www.cisco.com/en/US/solutions/collateral/ns341/ns525/ns537/ns705/ns827 /white paper_c11-520862.html

[111] Nortel Networks. (2006, Jan.). Supporting Real-time Traffic Preparing Your IP Network for Video Conferencing [Online]. Available: 
http://www.polycom.com/global/documents/whitepapers/supporting realtime traf fic preaparing ip network for_videoconferencing.pdf

[112] F. Kargl, E. Lawrence, M. Fischer, Y. Y. Lim, "Security, Privacy and Legal Issues in Pervasive eHealth Monitoring Systems," in Proc. 7th Int. Conf. on Mobile Business, Barcelona, Spain, July 2008, pp. 296-304.

[113] C. Karlof, N. Sastry, D. Wagner. TinySec: A Link Layer Security Architecture for Wireless Sensor Networks [Online]. Available: http://www.cs.berkeley.edu/ daw/papers/tinysec-sensys $04 . p d f$

[114] S. Saleem, S. Ullah, H. S. Yoo, "On the Security Issues in Wireless Body Area Networks," Journal of Digital Content Technology and its Applications (JDCTA), vol. 3, no. 3, pp. 178-184, Sept. 2009.

[115] A Kara, "Protecting Privacy in Remote-Patient Monitoring," IEEE Computer, vol. 34 , no. 5, pp. $24-27$, May 2001.

[116] Ontario Ministry of Health and Long Term Care. (2004). Personal Health Information Protection Act [Online]. Available: http://www.health.gov.on.ca/english/providers/legislation/priv_legislation/priv_le gislation.html

[117] Information and Privacy Commissioner of Ontario. (2009, Mar.). Innovative Wireless Home Care Services: Protecting Privacy and Personal Health Information [Online]. Available: http://www.ipc.on.ca/images/Resources/wirelesshomecare.pdf

[118] U.S. Department of Health and Human Services. Health Information Privacy [Online]. Available: http://www.hhs.gov/ocr/privacy/

[119] Texas Instruments Inc. (2007, Mar.). Single-Chip 2.4 GHz IEEE 802.15.4 Compliant and ZigBee ${ }^{\mathrm{TM}}$ Ready RF Transceiver [Online]. http://focus.ti.com/docs/prod/folders/print/cc2420.html

[120] D. Bhatia, L. Estevez, and S. Rao, "Energy Efficient Contextual Sensing for Elderly Care," in Proc. $28^{\text {th }}$ Int. Conf. IEEE Eng. Med. Biol. Soc., Lyon, France, 2009, pp. 4052-4055.

[121] G-Z Yang. Body Sensor Networks. Springer-Verlag, London, UK, 2006.

[122] R. O'Donnell and R. Perry. (2005, Nov.). Thin Computing ROI: The Untold Story [Online]. Available: http://www.wyse.com/resources/whitepapers/PDF/IDC ROI.pdf 
[123] F Troni, Mark A. Margevicius, M. A. Silver. (2008, Aug.). Total Cost of Ownership Comparison of PCs With Server-Based Computing [Online]. Available:

http://www.citrix.com/site/resources/dynamic/news/Gartner_SBC_TCO_Research . pdf

[124] J. W. Rittinghouse and J. F. Ransome. (2010). Cloud Computing Implementation, Management, and Security. CRC Press Taylor \& Francis Group 6000, Boca Raton, FL.

[125] M. O'Connell. (2010, Oct.). New developer Works survey shows dominance of cloud computing and mobile application development [Online]. Available: http://public.dhe.ibm.com/software/dw/survey/2010survey-results/2010surveyresults-pdf.pdf

[126] Livinlavidatech. (2010, Aug. 18). Cloud Computing: The Path To Skynet? [Online]. Available: http://livinlavidatech.wordpress.com/2010/08/18/cloudcomputing-the-path-to-skynet/

[127] A W. Kushniruk and V. L. Patel, "Cognitive and usability engineering methods for the evaluation of clinical information systems," Journal of Biomedical Informatics, vol. 37, no. 1, pp. 56-76, Feb. 2004.

[128] A. Arcelus, C. L. Herry, R. A. Goubran, F. Knoefel, H. Sveistrup, and M. Bilodeau, "Determination of Sit-to-Stand Transfer Duration Using Bed and Floor Pressure Sequences," IEEE Trans. Biomed. Eng., vol. 56, no. 10, pp. 2485-2492, Oct. 2009.

[129] A. Arcelus, I. Veledar, R. Goubran, F. Knoefel, H. Sveistrup, and M. Bilodeau, "Measurements of sit-to-stand timing and symmetry from bed pressure sensors," IEEE Trans. Instrum. Meas. May 2011.

[130] M. Holtzman, A. Arcelus, R. Goubran, and F. Knoefel, "Breathing signal fusion in pressure sensor arrays," in Proc. IEEE Int. Workshop Medical Measurement and Application, Ottawa, ON, 2008, pp. 71-76.

[131] M. H. Jones, R. A. Goubran, and F. Knoefel, "Identifying movement onset times for a bed-based pressure sensor array," in Proc. IEEE Int. Workshop Medical Measurement and Application, Benevento, Italy, 2006, pp. 111-114.

[132] W. Janssen, S. Külcü, H. Horemans, H. Stam, and J. Bussmann, "Sensitivity of accelerometry to assess balance control during sit-to-stand movement," IEEE Trans. Neural Syst. Rehabil. Eng., vol. 16, no. 5, pp. 479-484, Oct. 2008. 
[133] M. A. Dew, C. C. Hoch, D. J. Buysse, T. H. Monk, A. E. Begley, P. R. Houck, M. Hall, D. J. Kupfer, and C. F. Reynolds. 3rd, "Healthy older adults' sleep predicts all-cause mortality at 4 to 19 years of follow-up," Psychosomatic Med., vol. 65, no. 1, pp. 63-73, Feb. 2003.

[134] A. Polze, P. Tr"oger, U. Hentschel and T. Heinze, "A scalable, self-adaptive architecture for remote patient monitoring," 13th IEEE Int. Symposium on Object/Component/Service-Oriented Real-Time Distributed Computing Workshops, Carmona, Sevilla, Spain, May 2010, pp. 204-210.

[135] I. Mohomed, A. Misra, M. Ebling and W. Jerome, "HARMONI: Context-aware Filtering of Sensor Data for Continuous Remote Health Monitoring," in Proc. of $6^{\text {th }}$ Int. Conf. IEEE Pervasive Computing and Communications, Hong Kong 2008, pp. 248-251

[136] B. Mathieu, Y. Carlinet, D. Massaloux, B. Kövesi, and D. Deleam, "A NetworkAware Truncating Module for Scalable Streams Saving Bandwidth for Overused Networks," in Proc. of Second International Workshop on Mobility Aware Technologies and Applications, Montreal, Canada, Oct. 2005, pp. 12-21.

[137] A. D. Doulamis, D. I. Kosmopoulos, N. D. Doulamis, "Content-Based Time Sampling for Efficient Video Delivery over Networks of Low and Variable Bandwidth," in Proc. of the Int. Conf. on Digital Telecommunications ICDT, Cote d'Azur, France, Aug. 2006, pp. 27-27.

[138] H. Schwarz, D. Marpe, and T. Wiegand, "Overview of the scalable video coding extension of the H.264/AVC standard," IEEE Trans. Circuits Syst. Video Technol., vol. 17, no. 9, pp. 1103-1120, Sept. 2007.

[139] A.F. Abdelzaher and N. Bhatti, "Web Server QoS Management by Adaptive Content Delivery," Seventh IEEE Workshop on Quality of Service, London, UK, June 1999, pp. 216-225.

[140] S. Rho, J. Cho, and E. Hwang, "Adaptive Multimedia Content Delivery in Ubiquitous Environments." in Proc. of Int. Workshops Web Information Systems Engineering - WISE 2005, New York, NY, USA, Nov. 2005, pp. 43-52.

[141] A. T. Barth, M. A. Hanson, H. C. Powell Jr., and J. Lach, "Online Data and Execution Profiling for Dynamic Energy-Fidelity Optimization in Body Sensor Networks," Int. Conf. IEEE Body Sensor Networks, Singapore, June 2010, pp. 213-218. 
[142] K. Hua, H. Wang, W. Wang and S. Wu, "Adaptive Data Compression in Wireless Body Sensor Networks," To appear in Proc. of 13th Int. Conf. IEEE on Computational Science and Engineering, Hong Kong SAR, China, Dec. 2010.

[143] E. Ekmekcioglu, S. T. Worrall, A. M. Kondoz, "Bit-Rate Adaptive Downsampling for the Coding of Multi-View Video with Depth Information," in Proc. of IEEE 3DTV-Conference: The True Vision - Capture, Transmission and Display of 3D Video (3DTV-CON), Istanbul Turkey, May 2008, pp. $137-140$.

[144] S. Nasir, C.T.E.R. Hewage, M. Mrak, S. Worrall and A.M. Kondoz, "Depth based object prioritisation for 3D video communication over Wireless LAN," in Proc. of $16^{\text {th }}$ Int. Conf. IEEE on Image Processing (ICIP), Cairo Egypt, Nov. 2009, pp. $4269-4272$. 